\title{
Transmit Precoded Spatial Modulation: Maximizing the Minimum Euclidean Distance Versus Minimizing the Bit Error Ratio
}

\author{
Ping Yang, Yong Liang Guan, Member, IEEE, Yue Xiao, Marco Di Renzo, Senior Member, IEEE, \\ Shaoqian Li, Senior Member, IEEE, and Lajos Hanzo, Fellow, IEEE
}

Abstract-In this paper, we investigate a pair of transmit precoding (TPC) algorithms conceived for spatial modulation (SM) systems communicating over flat-fading multiple-input multiple-output (MIMO) channels. In order to retain all the benefits of conventional SM, we design the TPC matrix to be diagonal and introduce two design criteria for optimizing the elements of the TPC matrix. Specifically, we first investigate a TPC design based on maximizing the minimum Euclidean distance $d_{\min }\left(\max -d_{\min }\right)$ between the SM signal points at the receiver side. A closed-form solution of the optimal max- $d_{\min }$-based TPC matrix is derived. Then, another TPC design algorithm is proposed for directly minimizing the bit error ratio (BER) upper bound of SM, which is capable of jointly optimizing the overall Euclidean distance between all received signal points. In the minimum BER (min-BER)-based TPC algorithm, the theoretical gradient of the BER with respect to the diagonal TPC matrix is derived and a simplified iterative conjugate gradient (SCG) algorithm is invoked for TPC optimization. Our simulation results demonstrate that the proposed max- $d_{\min }$-based TPC algorithm is optimal in terms of the minimum distance. However, increasing $d_{\min }$ does not achieve a further BER improvement. We also confirm that the min-BERbased TPC outperforms the max- $d_{\text {min }}$-based TPC schemes in terms of the achievable BER performance.

Manuscript received March 23, 2015; revised November 2, 2015; accepted November 2, 2015. This work was supported in part by the National Science Foundation of China under Grant 61471090, in part by the National Basic Research Program of China under Grant 2013CB329001, in part by the National High-Tech R\&D Program of China ("863" Project under Grant 2014AA01A707), in part by the Foundation Project of National Key Laboratory of Science and Technology on Communications under Grant 9140C020108140C02005, and in part by the European Research Council's Advanced Fellow Grant are gratefully acknowledged. The work of M. D. Renzo was supported by the European Commission under the auspices of the FP7-PEOPLE MITN-CROSSFIRE project (grant 317126). The associate editor coordinating the review of this paper and approving it for publication was Z. Wang.

P. Yang is with the National Key Laboratory of Science and Technology on Communications, University of Electronic Science and Technology of China, Sichuan 611731, China, and also with the School of Electrical and Electronic Engineering, Nanyang Technological University, Singapore (e-mail: yang.ping@uestc.edu.cn).

Y. L. Guan is with the School of Electrical and Electronic Engineering, Nanyang Technological University, Singapore (e-mail: eylguan@ntu.edu.sg).

Y. Xiao and S. Li are with the National Key Laboratory of Science and Technology on Communications, University of Electronic Science and Technology of China, Sichuan 611731, China (e-mail: xiaoyue@uestc.edu.cn; lsq@yeste.edu.cn).

M. D. Renzo is with the Laboratory of Signals and Systems (L2S), University of Paris-Sud XI, Orsay, France (e-mail: marco.direnzo@1ss.supelec.fr)

L. Hanzo is with the School of Electronics and Computer Science, University of Southampton, Southampton SO17 1BJ, U.K. (e-mail: 1h@ecs.soton.ac.uk).

Color versions of one or more of the figures in this paper are available online at http://ieeexplore.ieee.org.

Digital Object Identifier 10.1109/TWC.2015.2497692
Index Terms-Minimum Euclidean distance, minimum BER, multiple-input multiple-output, pre-coding technique, spatial modulation.

\section{INTRODUCTION}

$\mathbf{R}$ ECENTLY, spatial modulation (SM) has been proposed as a new class of low-complexity energy-efficient multiple-input multiple-output (MIMO) approach, whilst relying on a single-radio frequency (RF) chain [1]-[3]. SM scheme relies on the unique encoding philosophy of activating one out of $N_{t}$ transmit antennas (TAs) during each transmission slot [4]. The activated TA then transmits classic complex-valued symbols of amplitude and phase modulation (APM). The potential benefits of SM over the conventional MIMO techniques have been validated not only via numerical simulations [5], [6] but also by laboratory experiments [7], [8].

Early work has been focused on low-complexity receiver designs conceived for minimizing the bit error ratio (BER) of SM [9]-[12]. It was shown in [9] that a low-complexity single-stream maximum likelihood (ML) detector or an even lower-complexity near-ML detector [10]-[12] is capable of striking a beneficial trade-off amongst the potentially conflicting factors of energy-efficiency, multiplexing gain and diversity gain compared to other MIMO transmission techniques [13]. In addition to a plethora of receivers, preprocessing at the transmitter has also been conceived for achieving a further performance improvement. Specifically, several antenna selection (AS) methods [14], [15] originally designed for conventional MIMO systems have also been generalized for employment in SM systems with the goal of enhancing its capacity or BER performance [16]-[18]. In [19]-[21], an adaptive SM (ASM) scheme was proposed for improving the achievable BER, while maintaining the target throughput with the aid of adaptive modulation (AM) techniques. In [22], the power allocation between the pilot and data was optimized for maximizing the capacity of SM transmission. In [23] and [24], a specific constellation design was proposed for space shift keying (SSK) systems in order to improve their BER. The constellation design was further developed in [25] for SM by finding the optimal combination of the number of TAs and the APM size that minimizes the BER.

Among the promising design alternatives, linear transmit pre-coding (TPC) techniques constitute an attractive transmit preprocessing regime, since they use a simple matrix $\mathbf{U}$ for 


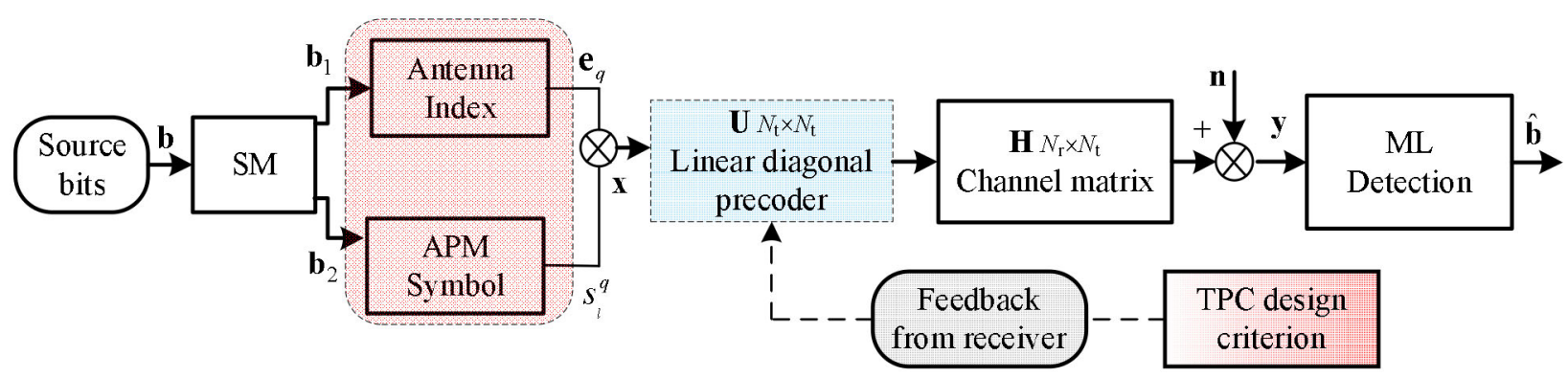

Fig. 1. The diagonal TPC based SM transmission system.

weighting the channel matrix in order to enhance the attainable performance [26]. Indeed, TPC has been widely researched in the context of classic spatial multiplexing systems [27]. However, since only a single TA is activated in each time slot in SM, these TPC approaches are not directly suitable for SM systems.

In [28] the effect of power imbalance has been researched in the context of SSK associated with TPC algorithms. More recently, the research efforts have been focused on the TPC design of SM based on maximizing the minimum Euclidean distance $d_{\min }\left(\max -d_{\min }\right)$ in the received SM constellation. In [29], the phase alignment technique has also been extended to SM systems for constellation shaping in order to provide BER benefit in multiple-input single-output (MISO) channels. In [30], the max- $d_{\min }$ based TPC is designed by using an iterative concave-convex process, where the TPC parameters are calculated for each transmit constellation points. In [31], [32], a diagonal TPC was proposed for maximizing $d_{\min }$ in SM systems. However, an exhaustive numerical search method was used for identifying the specific TPC parameters. On the other hand, in [33], a special case of the diagonal TPC, namely an adaptive power allocation (PA) method, was investigated, where a simple real-valued diagonal TPC matrix was considered. As shown in [33], closed-form solutions of the optimal max $-d_{\min }$ aided PA were derived in the case of two TAs. In [34], another diagonal TPC method, namely phase rotation precoding (PRP), was proposed for energy-efficient transmission, where only the phases of the SM symbols were optimized based on the $\max -d_{\min }$ criterion. The corresponding closed-form solutions were derived for two TAs and for PSK-modulated SM schemes in [35].

Against this background, the novel contributions of this paper are as follows.

1) We first investigate a general TPC matrix design algorithm based on the max- $d_{\min }$ criterion, where a complexvalued TPC matrix is considered instead of the realvalued PA matrix of [33] and the constant-modulus PRP matrix of [34], [35]. Compared to the heuristic method of computing the TPC matrix of [31], in this paper we derive closed-form solutions of the max- $d_{\min }$ TPC for a $\left(2 \times N_{r}\right)$-element BPSK-modulated SM scheme as well as for the more general cases of $M$-PSK modulated $(2 \times$ $N_{r}$ )-element SM. Moreover, we extend this method to the case of $N_{t}>2$.

2) It is shown that the max- $d_{\min }$ based TPC-aided SM scheme is capable of achieving a larger $d_{\min }$ than other max- $d_{\min }$ aided adaptive SM schemes, such as the PA 118 based TPC-aided SM scheme of [33] and the ASM 119 scheme of [21]. However an increase of $d_{\min }$ does not 120 achieve a further BER improvement. We find the reason 121 that the $\max -d_{\min }$ TPC only has a higher the mini- 122 mum received distance $d_{\min }$ and may result in reduced 123 Euclidean distances between the non-adjacent received 124 constellation points.

3) To alleviate this shortcoming, we propose a new 126 minimum-BER (min-BER) based TPC method, which is 127 capable of jointly optimizing the overall Euclidean dis- 128 tance between the received signal points. Specifically, the 129 theoretical gradient of the BER upper bound of SM with 130 respect to the diagonal TPC matrix $\mathbf{U}$ is derived, and the 131 simplified conjugate gradient (SCG) algorithm [36], [37] 132 is invoked for efficient TPC optimization. We demon- 133 strate that the overall BER gain of the proposed method 134 is significantly improved compared to both that of con- 135 ventional SM and to the other existing TPC schemes of 136 [29]-[35]. We also extend the proposed algorithm to cope 137 with channel state information (CSI) inaccuracies. 138

The organization of the paper is as follows. Section II intro- 139 duces the concept as well as the system model of the TPC-based 140 SM. In Section III and Section IV, we present a pair of TPC 141 designs conceived for enhancing the BER performance of SM. 142 The complexity analysis results are provided in Section V. Our 143 Simulation results and performance comparisons are presented 144 in Section VI. Finally, Section VII concludes the paper. 145

Notation: $(\cdot)^{*},(\cdot)^{T}$ and $(\cdot)^{H}$ denote conjugate, transpose, and 146 Hermitian transpose, respectively. The probability of an event is 147 represented by $P(\cdot)$. Furthermore, $\|\cdot\|$ stands for the Frobenius 148 norm and all logarithms are base of $2 . \operatorname{Tr}(\cdot)$ denotes the trace of 149 a square matrix, $E(\cdot)$ represents expectation, while $\operatorname{Re}\{\mathbf{x}\}$ and 150 $\operatorname{Im}\{\mathbf{x}\}$ represent the real and imaginary parts of $\mathbf{x}$, respectively. 151 $\mathbf{I}_{b}$ denotes a $(b \times b)$-element identity matrix and the operator 152 $\operatorname{diag}\{\cdot\}$ to be applied to a length $i$ vector returns an $i \times i$ square 153 matrix with the vector elements along the diagonal.

\section{SySTEM MOdeL}

\section{A. Signal Model of the Diagonal TPC Aided SM-MIMO}

Consider a MIMO system having $N_{t}$ transmit and $N_{r}$ receive 157 antennas. In this paper, $N_{t}$ is assumed to be a power of two. 158 Let $\mathbf{b}=\left[b_{1}, \ldots, b_{L}\right]$ be the transmit bit vector of each time 159 slot, which contains $L=\log _{2}\left(N_{t} M\right)$ bits. As shown in Fig. 1, 160 the input vector $\mathbf{b}$ is divided into two sub-vectors of $\log _{2}\left(N_{t}\right) \quad 161$ 
and $\log _{2}(M)$ bits, denoted as $\mathbf{b}_{1}$ and $\mathbf{b}_{2}$, respectively. The bits in the sub-vector $\mathbf{b}_{1}$ are used for selecting a unique TA index $q$ for activation, which is mapped to a $N_{t}$-dimensional standard basis vector $\mathbf{e}_{q}\left(1 \leq q \leq N_{t}\right)$. The bits in the sub-vector $\mathbf{b}_{2}$ are mapped to a Gray-coded APM symbol $s_{m}^{q} \in \mathfrak{I}(m \in$ $\{1, \ldots, M\})$ [38]. Then, the resultant SM symbol $\mathbf{x} \in \mathbb{C}^{N_{t} \times 1}$ can be formulated as [1]

$$
\mathbf{x}=s_{m}^{q} \mathbf{e}_{q}=\left[0, \ldots, s_{m}^{q}, \ldots, 0\right] .
$$

As shown in Fig. 1, after TPC relying on the linear diagonal matrix $\mathbf{U}$, the signal observed at the $N_{r}$ receive antennas is given by

$$
\mathbf{y}=\mathbf{H U x}+\mathbf{n},
$$

where $\mathbf{H}$ is the $\left(N_{r} \times N_{t}\right)$-element channel matrix, $\mathbf{U}$ is the $\left(N_{t} \times N_{t}\right)$-element TPC matrix, and $\mathbf{n}$ is the $\left(N_{r} \times 1\right)$-element noise vector. We assume $E\left[\mathbf{n n}^{H}\right]=N_{0} \mathbf{I}_{N_{r}}$ and $E\left[\mathbf{x n}^{H}\right]=$ $\mathbf{0}_{N_{t} \times N_{t}}$. The elements of the noise vector $\mathbf{n}$ are complex Gaussian random variables obeying $\mathcal{C N}\left(0, N_{0}\right)$. Furthermore, the diagonal TPC matrix $\mathbf{U}$ is given by

$$
\mathbf{U}=\operatorname{diag}\left\{u_{1}, \ldots, u_{q}, \ldots, u_{N_{t}}\right\},
$$

where $u_{q}$ is a complex-valued TPC parameter, which controls the channel gain associated with $x_{q}$. We enforce the constraint $\sum_{q=1}^{N_{t}}\left|u_{q}\right|^{2}=P_{\mathrm{T}}$ for the sake of normalizing the transmit power. The diagonal structure of $\mathbf{U}$ guarantees that the transmit vector $\mathbf{U x}$ has a single non-zero component, hence the single-RF-chain benefits (such as the avoidance of both the inter antenna interference (IAI) and of the multiple RFs) of SM are preserved. The matrix $\mathbf{U}$ can be decomposed as follows [31]

$$
\mathbf{U}=\mathbf{P} \Theta=\operatorname{diag}\left\{p_{1}, p_{2} e^{j \theta_{1}}, \ldots, p_{q} e^{j \theta_{q-1}}, \ldots, p_{N_{t}} e^{j \theta_{N_{t}-1}}\right\},
$$

where $u_{q}=p_{q} e^{j \theta_{q-1}}$ and $p_{q}$ represents the complex modulus of $u_{q}$, while $\theta_{q-1}$ represents the phase angle of $u_{q}$. In (4), the TPC matrix $\mathbf{U}$ is decomposed into two matrices related to the modulus and phase, which correspond to the real-valued PA matrix $\mathbf{P}=\operatorname{diag}\left\{p_{1}, p_{2}, \ldots, p_{q}, \ldots, p_{N_{t}}\right\}$ and to the complexvalued PRP matrix $\boldsymbol{\Theta}=\operatorname{diag}\left\{1, e^{j \theta_{1}}, \ldots, e^{j \theta_{q-1}}, \ldots, e^{j \theta_{N_{t}-1}}\right\}$, respectively.

Remark: The max- $d_{\min }$ based PA-aided SM schemes of [33] and the PRP-aided SM schemes of [34], [35] constitute special cases of the proposed TPC schemes, which can be obtained by setting $\boldsymbol{\Theta}=\mathbf{I}_{N_{t}}$ and $\mathbf{P}=\mathbf{I}_{N_{t}}$, respectively.

\section{B. Maximum Likelihood Receiver}

The receiver performs ML detection over all possible SM symbols $\mathbf{x} \in \mathbb{C}^{N_{t} \times 1}$ for retrieving the transmit symbols, which can be formulated as [9], [10]:

$$
\begin{aligned}
\hat{\mathbf{x}} & =\arg \min _{\mathbf{x} \in \mathbb{X}}\|\mathbf{y}-\mathbf{H U} \mathbf{x}\|^{2} \\
& =\arg \min _{\mathbf{x} \in \mathbb{X}}\|\mathbf{y}-\tilde{\mathbf{H}} \mathbf{x}\|^{2}=\arg \min _{q \in\left\{1, \ldots, N_{t}\right\}}\left\|\mathbf{y}-\tilde{\mathbf{h}}_{q} s_{m}^{q}\right\|^{2} \\
& \Leftrightarrow \arg \min _{q \in\left\{1, \ldots, N_{t}\right\}}\left\|\tilde{\mathbf{h}}_{q}^{H} \mathbf{y} /\right\| \tilde{\mathbf{h}}_{q}\left\|^{2}-s_{m}^{q}\right\|^{2},
\end{aligned}
$$

where $\mathbb{X}$ is the set of all legitimate transmit symbols and $\tilde{\mathbf{h}}_{q}$ is 201 the $q-t h$ column of the equivalent channel matrix $\tilde{\mathbf{H}}=\mathbf{H U} . \quad 202$ As shown in Eq. (5), a low-complexity single-stream ML detec- 203 tor is obtained [10], [22]. Moreover, it is shown in Proposition 204 1 of [22] that for a square- or for a rectangular-QAM constella- 205 tion, the complexity imposed is independent of the constellation 206 size, and that it increases only with $N_{t}$. 207

The conditional error performance of a ML receiver for 208 a given channel $\mathbf{H}$ can be approximated by the sum of the 209 pairwise error probability (PEP) [39], which is given by 210

$$
P(\mathbf{H}) \leq \frac{1}{L} \sum_{i=1}^{L} \sum_{\substack{j=1, i \neq j}}^{L} Q\left(\sqrt{\frac{1}{2 N_{0}} d_{i j}(\mathbf{H})}\right),
$$

where $Q(x)=(1 / \sqrt{2 \pi}) \int_{x}^{\infty} e^{-y^{2} / 2} d y$ denotes the Gaussian 211 tail probability, while the distance $d_{i j}(\mathbf{H})$ at the receiver is 212 defined as

$$
\begin{aligned}
d_{i j}(\mathbf{H}) & =\left\|\mathbf{H U}\left(\mathbf{x}_{i}-\mathbf{x}_{j}\right)\right\|^{2} \\
& =\left\|\mathbf{H} \mathbf{U} \mathbf{e}_{i j}\right\|^{2},
\end{aligned}
$$

where $\mathbf{e}_{i j}=\mathbf{x}_{i}-\mathbf{x}_{j}, i \neq j$ denotes the error vector. The PEP 214 depends on the specific SM symbol pair $\left(\mathbf{x}_{i}, \mathbf{x}_{j}\right)$, on the instan- 215 taneous channel realization $\mathbf{H}$ and the TPC matrix $\mathbf{U}$.

\section{Minimum Euclidean Distance Based TPC}

It follows by direct inspection of the PEP expression of 218 Eq. (6) that the performance of the ML receiver is pre- 219 dominantly affected by the distances $d_{i j}(\mathbf{H})$. Motivated by 220 this observation, TPC design methods based on maximiz- 221 ing the minimum value of $d_{i j}(\mathbf{H})$ (the distance $d_{\min }$ ) have 222 been introduced in [31] and [32]. However, only a high- 223 complexity numerical approach was proposed for optimizing 224 the TPC matrix. In this section, we first briefly introduce the 225 max $-d_{\min }$ based TPC method. Then, we derive the related 226 solutions.

\section{A. Design Criterion}

At high SNR, Eq. (6) can be further simplified as follows [39]

229

$$
P(\mathbf{H}) \leq \lambda \cdot Q\left(\sqrt{\frac{1}{2 N_{0}} d_{\min }}\right),
$$

where $\lambda$ is the number of neighbor points [39] and $d_{\min }$ is 230 defined as

$$
d_{\min }=\min _{\substack{i, j \\ i \neq j}} d_{i j}(\mathbf{H})=\min _{\mathbf{e}_{i j} \in \mathbb{E}}\left\|\mathbf{H U} \mathbf{e}_{i j}\right\|^{2} .
$$

In Eq. (8), $P(\mathbf{H})$ is a monotonically decreasing function of 232 $d_{\min }$. Hence, the system's BER performance may be improved 233 by maximizing the distance $d_{\min }$ of the received constellation 234 upon carefully adapting the TPC matrix $\mathbf{U}$ under the power 235 
constraint $P_{\mathrm{T}} .{ }^{1}$ Based on this principle, the $\max -d_{\min }$ based TPC matrix $\mathbf{U}$ design rule can be formulated as follows

$$
\begin{aligned}
& \mathbf{U}_{\text {opt }}=\underset{\mathbf{U}}{\arg \max } d_{\min } \\
& \text { s.t. } \quad \operatorname{tr}\left(\mathbf{U U}^{T}\right) \leq P_{\mathrm{T}} .
\end{aligned}
$$

Note that in [33] and [35] the closed-form solutions for two special cases of Eq. (10), namely for the $\max -d_{\min }$ based PA matrix and for the max $-d_{\min }$ based PRP matrix, have been derived. However, to the best of our knowledge, the closedform solution for the joint design of the PA and PRP of Eq. (10) has not been reported in the existing literature. In the following subsections, we derive a closed-form solution for the TPC matrix of the BPSK-modulated $\left(2 \times N_{r}\right)$-element SM and extend the method to the more general $M$-PSK modulated $\left(2 \times N_{r}\right)$-element SM arrangements. Additionally, as shown in [2] and [6], PSK schemes are preferred over QAM schemes in SM. Hence, PSK is adopted in this paper.

\section{B. Optimal TPC Matrix for BPSK-modulated $2 \times N_{r} S M$}

Let us consider a BPSK-modulated SM systems associated with $N_{t}=2$, where the BPSK symbols belong to the set $\mathfrak{s}=\{1,-1\}$, and all possible error vectors $\mathbf{e}_{i j}=\mathbf{x}_{i}-\mathbf{x}_{j}, i \neq$ $j$ are listed as follows: $\left\{[-2,0]^{T},[2,0]^{T},[0,-2]^{T},[0,2]^{T}\right.$, $\left.[-1,1]^{T},[-1,-1]^{T},[1,-1]^{T},[1,1]^{T}\right\}$. Similar to the method of [33], since some vectors are collinear, the set to be studied is reduced to $\tilde{\mathbb{E}}_{B P S K}=\left\{\mathbf{e}_{1}, \mathbf{e}_{2}, \mathbf{e}_{3}, \mathbf{e}_{4}\right\}=\left\{[2,0]^{T},[0,2]^{T}\right.$, $\left.[1,-1]^{T},[1,1]^{T}\right\}$. Given the channel matrix $\mathbf{H}=\left[\mathbf{h}_{1}, \mathbf{h}_{2}\right]$ and the corresponding TPC matrix $\mathbf{U}=\operatorname{diag}\left\{p_{1}, p_{2} e^{j \theta_{1}}\right\}$, the distances at the receiver based on Eq. (7) are given by

$$
\left\{\begin{aligned}
d_{1} & =\left\|\mathbf{H U} \mathbf{e}_{1}\right\|^{2}=4\left\|p_{1} \mathbf{h}_{1}\right\|^{2} \\
d_{2} & =\left\|\mathbf{H U} \mathbf{e}_{2}\right\|^{2}=4\left\|p_{2} \mathbf{h}_{2}\right\|^{2} \\
d_{3} & =\left\|\mathbf{H U} \mathbf{e}_{3}\right\|^{2}=\left\|p_{1} \mathbf{h}_{1}-p_{2} e^{j \theta_{1}} \mathbf{h}_{2}\right\|^{2} \\
d_{4} & =\left\|\mathbf{H U} \mathbf{e}_{4}\right\|^{2}=\left\|p_{1} \mathbf{h}_{1}+p_{2} e^{j \theta_{1}} \mathbf{h}_{2}\right\|^{2}
\end{aligned}\right.
$$

Based on the distances in Eq. (11), the optimization problem of Eq. (10) can be modified as follows

$$
\begin{aligned}
& \mathbf{U}_{\mathrm{opt}}=\underset{\mathbf{U}}{\arg \max }\left\{\min \left\{d_{1}, d_{2}, d_{3}, d_{4}\right\}\right\} \\
& \text { s.t. } \quad \operatorname{tr}\left(\mathbf{U} \mathbf{U}^{T}\right) \leq P_{\mathrm{T}}
\end{aligned}
$$

To obtain the specific TPC matrix $\mathbf{U}_{\mathrm{opt}}$, which maximizes the distance $d_{\min }$, the parameters $p_{1}, p_{2}$ and $\theta_{1}$ in Eq. (12) have to be computed. As indicated in Eq. (11) and shown in Fig. 2, for a fixed PA matrix $\mathbf{P}=\operatorname{diag}\left\{p_{1}, p_{2}\right\}, d_{1}$ and $d_{2}$ are independent of the phase $\theta_{1}$, while $d_{3}$ and $d_{4}$ are given by sinusoidal functions of the phase $\theta_{1}$. In order to find the optimal phase solution $\theta_{1}^{o p t}$, we can first obtain the phases assigned to

\footnotetext{
${ }^{1}$ Compared to the PRP method of [34], [35], the power of the SM symbols may indeed fluctuate due to the TPC algorithm. However, during the time when the channel envelope remains constant within its coherence-interval, the power values of the transmit symbols are selected from a finite discrete set. In practice, the constraint $P_{\mathrm{T}}$ should be carefully selected according to the system requirements, such as the peak to average power ratio (PAPR) and the BER metrics.
}

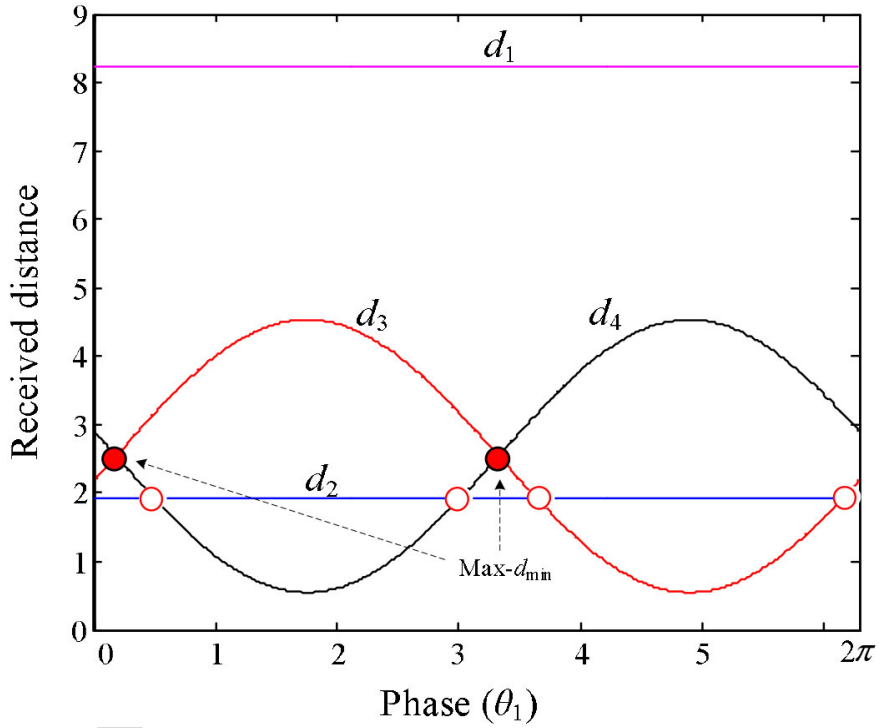

Fig. 2. The phase solutions of the BPSK-modulated $\left(2 \times N_{r}\right)$-element TPC based SM for a fixed PA.

the TAs by finding the intersections of the sinusoidal curves in 270 Fig. 2, and then continue by computing the optimal PA matrix 271 as $\mathbf{P}=\operatorname{diag}\left\{p_{1}^{o p t}, p_{2}^{o p t}\right\}$.

To be specific, as shown in Fig. 2, regardless of the specific 273 PA matrix $\mathbf{P}$, the optimal phase $\theta_{1}^{\text {opt }}$ that maximizes $d_{\min }$ should 274 satisfy the constraint of $d_{3}=d_{4}$ which from (11):

$$
\left\|p_{1} \mathbf{h}_{1}-p_{2} e^{j \theta_{1}^{o p t}} \mathbf{h}_{2}\right\|^{2}=\left\|p_{1} \mathbf{h}_{1}+p_{2} e^{j \theta_{1}^{o p t}} \mathbf{h}_{2}\right\|^{2} .
$$

Eq. (13) can be further simplified to $\operatorname{Re}\left\{\mathbf{h}_{1}^{H} \mathbf{h}_{2} e^{j \theta_{1}^{o p t}}\right\}=0.276$ Upon introducing the shorthands $a=\left\|\mathbf{h}_{1}\right\|^{2}, b=\left\|\mathbf{h}_{2}\right\|^{2}, c_{1}=277$ $2 \cdot \operatorname{Re}\left\{\mathbf{h}_{1}^{H} \mathbf{h}_{2}\right\}$ and $c_{2}=2 \cdot \operatorname{Im}\left\{\mathbf{h}_{1}^{H} \mathbf{h}_{2}\right\}$ for a given channel 278 matrix H, Eq. (13) can be solved as

$$
\begin{aligned}
& c_{1} \cos \theta_{1}^{o p t}-c_{2} \sin \theta_{1}^{o p t}=0 \\
& \Leftrightarrow \theta_{1}^{o p t}=k \pi+\tan ^{-1}\left(\frac{c_{1}}{c_{2}}\right), k \in \mathbb{Z}, 0 \leq \theta_{1}^{o p t} \leq 2 \pi .
\end{aligned}
$$

Given the phase solution of Eq. (14), the distances $d_{3}$ and $d_{4} 280$ of Eq. (11) are simplified as follows

$$
d_{3}=d_{4}=\left\|p_{1} \mathbf{h}_{1}\right\|^{2}+\left\|p_{2} \mathbf{h}_{2}\right\|^{2}=a p_{1}^{2}+b p_{2}^{2} .
$$

To compute the optimal PA parameters as $p_{1}^{o p t}$ and $p_{2}^{o p t}, 282$ since we have $d_{3}=d_{4}$ in Eq. (15), the optimization problem 283 of Eq. (12) can be further simplified to:

$$
\begin{aligned}
\mathbf{U}_{\mathrm{opt}}=\underset{\mathbf{U}}{\arg \max }\left\{\min \left\{d_{1}, d_{2}, d_{3}\right\}\right\} \\
=\underset{\mathbf{U}}{\arg \max }\left\{\operatorname { m i n } \left\{4 a p_{1}^{2}, 4 b\left(P_{\mathrm{T}}-p_{1}^{2}\right),\right.\right. \\
\left.\left.\quad a p_{1}^{2}+b\left(P_{\mathrm{T}}-p_{1}^{2}\right)\right\}\right\} .
\end{aligned}
$$

As indicated in Eq. (16), $d_{1}, d_{2}$ and $d_{3}$ are linear functions 285 of the parameter $\mu_{1}=p_{1}^{2}$. Hence, the max- $d_{\min }$ solution given 286 
$\mu_{1}$ is one of the intersections between these distances $d_{i}(i=$ $1,2,3)$, which are given by

$$
\left\{\begin{array}{l}
p_{1}^{(1)}=\sqrt{b /(a+b) P_{\mathrm{T}}} \\
p_{1}^{(2)}=\sqrt{b /(3 a+b) P_{\mathrm{T}}} \\
p_{1}^{(3)}=\sqrt{3 b /(3 b+a) P_{\mathrm{T}}}
\end{array},\right.
$$

where $p_{1}^{(i)}, i=1,2,3$ are the power assigned to the first TA for the $i$ th intersections. Then, based on the fixed total power constraint $P_{\mathrm{T}}$, the corresponding power assigned to the second TA is given by

$$
\left\{\begin{array}{l}
p_{2}^{(1)}=\sqrt{a /(a+b) P_{\mathrm{T}}} \\
p_{2}^{(2)}=\sqrt{3 a /(3 a+b) P_{\mathrm{T}}} \\
p_{2}^{(3)}=\sqrt{a /(3 b+a) P_{\mathrm{T}}}
\end{array}\right.
$$

Based on Eqs. (17) and (18), we select the one providing the maximum $d_{\min }$ as the final solution. Finally, the optimal TPC matrix $\mathbf{U}_{\mathrm{opt}}$ and the corresponding maximized $d_{\text {min }}$, namely $d_{\min }^{\max }$, are given by

$\left\{\begin{array}{lll}\text { if } a \geq b, & \mathbf{U}_{\mathrm{opt}}=\operatorname{diag}\left\{p_{1}^{(2)}, p_{2}^{(2)} e^{j \theta_{1}^{o p t}}\right\}, & d_{\min }^{\max }=\frac{4 a b}{3 a+b} \\ \text { if } a<b, & \mathbf{U}_{\mathrm{opt}}=\operatorname{diag}\left\{p_{1}^{(3)}, p_{2}^{(3)} e^{\left.j \theta_{1}^{\text {opt }}\right\},}\right. & d_{\min }^{\max }=\frac{4 a b}{3 b+a}\end{array}\right.$

It is worth noting that since the Euclidean distance $d_{\min }^{\max }$ of Eq. (19) has two independent channel gains, a transmit diversity order of two may be achieved [40].

\section{Optimal TPC Matrix for M-PSK-Modulated $\left(2 \times N_{r}\right)$ - Element SM}

In this subsection, the approach proposed in Section III-B is extended to $M$-PSK modulated SM schemes. Based on the method of Section III-B, the max- $d_{\min }$ based TPC algorithm can be summarized as follows:

In order to better illustrate the general algorithm described above, let us consider the specific example of constant-modulus $M$-PSK modulation, whose symbols belong to the set $s \sim \mathfrak{s}=$ $e^{j \frac{2 l \pi}{M}}(l \in\{1, \ldots, M\})$. The minimum distance between two symbols of the $M$-PSK constellation is $d_{M-\mathrm{PSK}}=2 \sin (\pi / M)$ [38]. Since the SM symbols $\mathbf{x}_{i}$ and $\mathbf{x}_{j}$ only have a single non-zero element, the error vectors $\mathbf{e}_{i j}=\mathbf{x}_{i}-\mathbf{x}_{j}, i \neq j$ can be classified into two types: the error vectors having only a single non-zero element, and those having two non-zero elements. The first type is generated by the transmit symbols $\mathbf{x}_{i}$ and $\mathbf{x}_{j}$ associated with the same TA activation position, while the second type is generated by the symbols having different active TAs. As a result, the distance $d_{i j}(\mathbf{H})$ of Eq. (7) can be divided into two sets: $\mathbb{D}_{1}$ and $\mathbb{D}_{2}$, which are given by

$$
\left\{\begin{array}{l}
\mathbb{D}_{1}=\left\{p_{i}^{2}\left\|\mathbf{h}_{i}\right\|^{2}\left(s_{l}-s_{\hat{l}}\right), l \neq \hat{l}, i=1,2\right\} \\
\mathbb{D}_{2}=\left\{\left\|p_{1} \mathbf{h}_{1} s_{l}-p_{2} \mathbf{h}_{2} s_{\hat{l}}\right\|^{2}, l, \hat{l}=1, \ldots M\right\}
\end{array},\right.
$$

where $s_{l}=e^{j \frac{2 l \pi}{M}}$ and $s_{\hat{l}}=e^{j \frac{2 \hat{l} \pi}{M}}$ are two $M$-PSK symbols. Since only the minimum distance is investigated in the max$d_{\text {min }}$ optimization problem of Eq. (10), only the minimum value
Algorithm 1. The max $-d_{\text {min }}$ based TPC algorithm

Step 1: Compute all legitimate error vectors $\mathbf{e}_{i j}=\mathbf{x}_{i}-\mathbf{x}_{j}, i \neq$ $j$ by eliminating all collinear elements. Calculate all legitimate received distances $d_{i j}(\mathbf{H})$ with the aid of the channel matrix $\mathbf{H}$ and $\mathbf{e}_{i j}$. Let $\mathbb{D}$ be the set of these distances, whose elements are denoted by $d_{v}(v=$ $1, \ldots, V)$, where $V$ is the cardinality of the set $\mathbb{D}$. The set $\mathbb{D}$ is divided into two sub-sets $\mathbb{D}_{1}$ and $\mathbb{D}_{2}$, where $\mathbb{D}_{1}$ contains the error vectors, which have only a single non-zero element, and $\mathbb{D}_{2}$ contains the error vectors, which have two non-zero elements. ${ }^{2}$

Step 2: Find the optimal phase $\theta_{1}^{o p t}$, which maximizes the minimum received distance of the set $\mathbb{D}_{2}$. Note that there may be multiple optimal phase solutions, which are calculated based on shifted sinusoidal functions $d_{v}$ in $\mathbb{D}_{2}$. Since these solutions provide the same $d_{\text {min }}$, any one of them can be randomly selected.

Step 3: After finding the optimal phase $\theta_{1}^{o p t}$, compute all possible intersections between the received distances $d_{i}$ and $d_{j}\left(d_{i}, d_{j} \in \mathbb{D}\right)$ and compute the corresponding PA matrix P. Select the one having the largest $d_{\text {min }}$ as the final PA result, which can be formulated as $\mathbf{P}=\operatorname{diag}\left\{p_{1}^{o p t}, p_{2}^{o p t}\right\}$. Then, the final TPC solution is given by $\mathbf{U}_{\text {opt }}=\operatorname{diag}\left\{p_{1}^{o p t}, p_{2}^{o p t} e^{j \theta_{1}^{o p t}}\right\}$.

of the set $\mathbb{D}_{1}$ has to be considered. To be specific, only the pair 322 of elements $d_{1}=\min _{l \neq \hat{l}} p_{1}^{2}\left\|\mathbf{h}_{1}\right\|^{2}\left(s_{l}-s_{\hat{l}}\right)=d_{M-\operatorname{PSK}} p_{1}^{2}\left\|\mathbf{h}_{1}\right\|^{2} 323$ and $d_{2}=\min _{l \neq \hat{l}} p_{2}^{2}\left\|\mathbf{h}_{2}\right\|^{2}\left(s_{l}-s_{\hat{l}}\right)=d_{M-\mathrm{PSK}} p_{2}^{2}\left\|\mathbf{h}_{2}\right\|^{2}$ has to be 324 considered in $\mathbb{D}_{1}$. Hence, the set $\mathbb{D}_{1}$ is simplified to

$$
\mathbb{D}_{1}=\left\{d_{M-\mathrm{PSK}}^{2} p_{1}^{2}\left\|\mathbf{h}_{1}\right\|^{2}, d_{M-\mathrm{PSK}}^{2} p_{2}^{2}\left\|\mathbf{h}_{2}\right\|^{2}\right\} .
$$

Let us reduce the set $\mathbb{D}_{2}$. When only the phase difference of 326 the PSK symbols $s_{l}=e^{j \frac{2 l \pi}{M}}$ and $s_{\hat{l}}=e^{j \frac{2 \hat{l} \pi}{M}}$ is considered, the 327 set $\mathbb{D}_{2}$ can be modified to

$$
\begin{aligned}
\mathbb{D}_{2} & =\left\{\left\|\mathbf{h}_{1} p_{1} s_{l}-\mathbf{h}_{2} p_{2} e^{j \theta_{1}} s_{\hat{l}}\right\|^{2}, s_{l}, s_{\hat{l}} \in \mathfrak{s}\right\} \\
& =\left\{\left\|p_{1} \mathbf{h}_{1} e^{j \frac{2(l-\hat{l}) \pi}{M}}-p_{2} e^{j \theta_{1}} \mathbf{h}_{2}\right\|^{2}, l, \hat{l}=1, \ldots M\right\} \\
& =\left\{\left\|p_{1} \mathbf{h}_{1} e^{j \frac{2 k \pi}{M}}-p_{2} e^{j \theta_{1}} \mathbf{h}_{2}\right\|^{2}, k=0, \ldots M-1\right\},
\end{aligned}
$$

where the phase difference factor is $k=l-\hat{l}$. The reduction 329 principle behind Eq. (22) is that if the error vectors in the set $\mathbb{D}_{2} 330$ having only a phase difference, they provide the same distance 331 at the receiver. Based on this principle, the number of elements 332 in $\mathbb{D}_{2}$ is reduced to $M$ compared to $M(M-1)$. 333

Let $\lambda_{k}=\frac{2 k \pi}{M}$ be the phase difference of the symbol $s_{l}$ and 334 $s_{\hat{l}}$. Since the distances in the set $\mathbb{D}_{1}$ are independent of the 335 phase $\theta_{1}$, similar to the BPSK case portrayed in Section III-B, 336

\footnotetext{
${ }^{2}$ Note that the transmit vector of SM has only a single non-zero element, hence the number of non-zero elements of the error vectors of SM is up to 2.
} 
it is possible to first find the optimal phase $\theta_{1}^{o p t}$, which maximizes the minimum received distance of the set $\mathbb{D}_{2}$. To achieve this goal, the intersections between arbitrary received distances $\left\|\mathbf{h}_{1} p_{1} e^{j \lambda_{a}}-\mathbf{h}_{2} p_{2} e^{j \theta_{1}}\right\|^{2}$ and $\left\|\mathbf{h}_{1} p_{1} e^{j \lambda_{b}}-\mathbf{h}_{2} p_{2} e^{j \theta_{1}}\right\|^{2}$ in Eq. (22) are firstly calculated as

$$
\begin{aligned}
& \left\|\mathbf{h}_{1} p_{1} e^{j \lambda_{a}}-\mathbf{h}_{2} p_{2} e^{j \theta_{1}}\right\|^{2}=\left\|\mathbf{h}_{1} p_{1} e^{j \lambda_{b}}-\mathbf{h}_{2} p_{2} e^{j \theta_{1}}\right\|^{2} \\
& \Leftrightarrow\left(c_{1} \cos \lambda_{a}-c_{2} \sin \lambda_{a}-c_{1} \cos \lambda_{b}+c_{2} \sin \lambda_{b}\right) \cos \theta_{1} \\
& =-\left(c_{1} \sin \lambda_{a}+c_{2} \cos \lambda_{a}-c_{1} \sin \lambda_{b}-c_{2} \cos \lambda_{b}\right) \sin \theta_{1} \\
& \Leftrightarrow \tan \theta_{1}=-\frac{c_{1} \cos \lambda_{a}-c_{2} \sin \lambda_{a}-c_{1} \cos \lambda_{b}+c_{2} \sin \lambda_{b}}{c_{1} \sin \lambda_{a}+c_{2} \cos \lambda_{a}-c_{1} \sin \lambda_{b}-c_{2} \cos \lambda_{b}} .
\end{aligned}
$$

After that, all possible optimal phase $\theta_{1}^{o p t}$ can be obtained from Eq. (23) as

$$
\left\{\begin{array}{c}
\theta_{1}^{o p t}=k \pi+\tan ^{-1}\left(\frac{-c_{1} \cos \lambda_{a}+c_{2} \sin \lambda_{a}+c_{1} \cos \lambda_{b}-c_{2} \sin \lambda_{b}}{c_{1} \sin \lambda_{a}+c_{2} \cos \lambda_{a}-c_{1} \sin \lambda_{b}-c_{2} \cos \lambda_{b}}\right), \\
k \in \mathbb{Z}, 0 \leq \theta_{1}^{o p t} \leq 2 \pi .
\end{array} .\right.
$$

Finally, the optimal phase is the candidate providing the maximum distance $d_{\min }$ in the set of Eq. (24). After computing the optimal phase, the the PA matrix can be optimized based on all possible intersections of $d_{v}(v=1, \ldots, V)$, similar to processes of Eqs. (16)-(18). Following these calculation steps, the optimal TPC matrix, which combines the optimal phase and PA parameters, is obtained in closed-form.

\section{Example for QPSK Modulation}

Based on the algorithm in Section III-C, we calculate the optimal TPC solution for QPSK-modulated $\left(2 \times N_{r}\right)$-element $\mathrm{SM}$, which will be used in our simulations. The symbols of QPSK modulation belong to the set $\mathfrak{s}=\{1,-1, j,-j\}$ and the value of $d_{4-\mathrm{PSK}}$ is equal to $\sqrt{2}$. Based on Eqs. (21) and (22), the corresponding sets $\mathbb{D}_{1}$ and $\mathbb{D}_{2}$ for QPSK modulation are

$$
\left\{\begin{array}{l}
\mathbb{D}_{1}=\left\{2 p_{1}^{2}\left\|\mathbf{h}_{1}\right\|^{2}, 2 p_{2}^{2}\left\|\mathbf{h}_{2}\right\|^{2}\right\} . \\
\mathbb{D}_{2}=\left\{\left\|p_{1} \mathbf{h}_{1} e^{j \frac{2 k \pi}{M}}-p_{2} e^{j \theta_{1}} \mathbf{h}_{2}\right\|^{2}, k=0, \ldots, 3\right\},
\end{array}\right.
$$

According to Eq. (24), there are two optimal phases $\theta_{1}^{o p t}$ that maximizes the distance $d_{\min }$ for Eq. (25), namely $\theta_{1}^{\text {opt }, 1}$ and $\theta_{1}^{\text {opt }, 2}$, which are given by

$$
\left\{\begin{array}{c}
\theta_{1}^{o p t, 1}=k \pi+\tan ^{-1}\left(\frac{-c_{1}-c_{2}}{c_{1}-c_{2}}\right) \\
k \in \mathbb{Z}, 0 \leq \theta_{1}^{o p t, 1} \leq 2 \pi
\end{array}\right.
$$

and

$$
\left\{\begin{array}{c}
\theta_{1}^{o p t, 2}=k \pi+\tan ^{-1}\left(\frac{c_{1}-c_{2}}{c_{1}+c_{2}}\right), \\
k \in \mathbb{Z}, 0 \leq \theta_{1}^{o p t, 2} \leq 2 \pi
\end{array}\right.
$$

Since both solutions $\theta_{1}^{o p t, 1}$ and $\theta_{1}^{o p t, 2}$ have the same $d_{\min }$, we consider only the first case $\theta_{1}=\theta_{1}^{o p t, 1}$. After finding the optimal phase $\theta_{1}^{o p t, 1}$, the received distance set $\mathbb{D}_{2}$ is further reduced to $\mathbb{D}_{2}=\left\{\left\|p_{1} \mathbf{h}_{1} e^{j \frac{2 k \pi}{M}}-p_{2} e^{j \theta_{1}} \mathbf{h}_{2}\right\|^{2}, k=0,1\right\}$. This reduction 365 is due to the fact that $\theta_{1}^{o p t, 1}$ corresponds to the intersection of 366 two elements of $\mathbb{D}_{2}$ and the elements having the same value are 367 eliminated. After this reduction, the final received distance set 368 has only 4 elements (both $\mathbb{D}_{1}$ and $\mathbb{D}_{2}$ have 2 elements), denoted 369 by $\bar{d}_{i}(i=1,2,3,4)$.

Given the optimal phase as $\theta_{1}^{\text {opt }}=\theta_{1}^{\text {opt }, 1}$, we have to fur- 371 ther identify the optimal PA parameters $p_{1}^{o p t}$ and $p_{2}^{o p t}\left(p_{2}^{o p t}=372\right.$ $\sqrt{\left.P_{\mathrm{T}}-\left(p_{1}^{o p t}\right)^{2}\right)}$. According to the step 3 of Algorithm 1, the 373 max $-d_{\min }$ solution of $p_{1}$ is one of the intersections between 374 these received distance $\bar{d}_{i}(i=1,2,3,4)$, which are given by 375

$$
\left\{\begin{array}{l}
p_{1}^{(1)}=\sqrt{b /(a+b) P_{\mathrm{T}}} \\
p_{1}^{(2)}=p_{1}^{(5)}=\sqrt{\frac{2 c^{2}+4 a b+2 \tilde{C} \sqrt{\tilde{C}^{2}+4 a b}}{4 a^{2}+2 c^{2}+4 a b+2 \tilde{C} \sqrt{\tilde{C}^{2}+4 a b}} P_{\mathrm{T}}} \\
p_{1}^{(3)}=p_{1}^{(4)}=\sqrt{\frac{2 c^{2}+4 a b-2 \tilde{C} \sqrt{\tilde{C}^{2}+4 a b}}{4 a^{2}+2 c^{2}+4 a b-2 \tilde{C} \sqrt{\tilde{C}^{2}+4 a b}} P_{\mathrm{T}}}
\end{array} .\right.
$$

Based on the power constraint, the power allocated on 376 the second $\mathrm{TA}$ is obtained by $p_{2}^{(i)}=\sqrt{P_{\mathrm{T}}-\left(p_{1}^{(i)}\right)^{2}}, i=377$ $1, \ldots, 5$. Finally, the distances $d_{\min }$ of these TPC solutions 378 $\mathbf{U}=\operatorname{diag}\left\{p_{1}^{(i)}, p_{2}^{(i)} e^{j \theta_{1}^{o p t, 1}}\right\}(i=1,2,3,4,5)$ are generated and 379 that having the largest $d_{\min }$ is chosen as our final result $\mathbf{U}_{\text {opt }}$. 380

E. A Low-Complexity Iterative Max- $d_{\min }$ for $N_{t}>2$

It is worth mentioning that the restriction of considering 382 $\left(2 \times N_{r}\right)$-element $\mathrm{SM}$ is imposed by the difficulty of the $d_{\min } 383$ optimization. The solution of the general problem remains an 384 open challenge for two reasons. Firstly, the solution depends on 385 both the channel matrix and on the symbol alphabet, and sec- 386 ondly, the solution space is large. Similar to the general method 387 proposed in the PA aided SM of [33], some sub-optimal meth- 388 ods can be adopted for the case of $N_{t}>2$ based on an iterative 389 process relying on the above-mentioned optimal max- $d_{\min }$ solu- 390 tion provided for $N_{t}=2$, where the TPC algorithm will only 391 be used for the specific TA pair associated with $d_{\min }$, while the 392 parameters of other TAs remain unchanged in each iteration.

\section{Minimum BER BASED TPC}

Although the max- $d_{\min }$ based TPC algorithm is simple, it 395 may not achieve a significant BER improvement for some SM 396 systems, because only one of the distances in the PEP expres- 397 sion of Eq. (6), namely $d_{\text {min }}$, is optimized at the receiver. 398 Moreover, we can only obtain closed-form solutions for this 399 TPC algorithm for the case of $N_{t}=2$. To deal with these prob- 400 lems, we propose a new min-BER based TPC algorithm, which 401 is capable of jointly optimizing all the received distances for 402 directly improving the BER for arbitrary value of $N_{t}$. By con- 403 sidering the bit-to-symbol mapping rule of our SM scheme, a 404 more accurate conditional BER bound based on Eq. (6) can be 405 obtained as [41]

$$
\begin{aligned}
P_{e}(\mathbf{H}) \leq P_{e}^{\text {up }}(\mathbf{H})= & \frac{1}{L} \sum_{\substack{\mathbf{x}_{i} \in \mathbb{X} \\
\mathbf{x}_{\mathbf{x}_{j} \in \mathbb{X}} \\
\mathbf{x}_{i} \neq \mathbf{x}_{j}}} D_{H}\left(\mathbf{x}_{i} \rightarrow \mathbf{x}_{j}\right) \\
& \cdot Q\left(\sqrt{\frac{1}{2 N_{0}}\left\|\mathbf{H U}\left(\mathbf{x}_{i}-\mathbf{x}_{j}\right)\right\|^{2}}\right),
\end{aligned}
$$


where $D_{H}\left(\mathbf{x}_{i} \rightarrow \mathbf{x}_{j}\right)$ is the Hamming distance between the SM signals $\mathbf{x}_{i}$ and $\mathbf{x}_{j}$. From Eq. (29), the min-BER-based TPC matrix is proposed by solving the optimization problem as follows

$$
\begin{aligned}
& \mathbf{U}_{\mathrm{opt}}=\underset{\mathbf{U}}{\arg \min } P_{e}^{\mathrm{up}}(\mathbf{H}) \\
& \text { s.t. } \operatorname{tr}\left(\mathbf{U}^{T}\right)=P_{\mathrm{T}}
\end{aligned}
$$

Remark: Compared to Eq. (30), the $\max -d_{\min }$ based TPC algorithm of Eq. (10) considers only a reduced summation over a subset of $\mathbb{X}$, which has the smallest Euclidean distance. Therefore, it can only minimize a much looser bound of BER than the bound of Eq. (29).

\section{A. Precoder Design Based on Gradient Optimization}

Since the direct solution of Eq. (30) is complex, we drive the theoretical gradient of the cost function with respect to the diagonal TPC matrix $\mathbf{U}$ and invoke the SCG algorithm of [36] for low-complexity TPC matrix optimization. More specifically, the cost function of the SCG algorithm is obtained from Eq. (29) and is defined as

$$
\begin{aligned}
J e(\mathbf{U})= & \sum_{\substack { \mathbf{x}_{i} \in \mathbb{X} \\
\begin{subarray}{c}{\mathbf{x}_{j} \in \mathbb{X} \\
\mathbf{x}_{i} \neq \mathbf{x}_{j}{ \mathbf { x } _ { i } \in \mathbb { X } \\
\begin{subarray} { c } { \mathbf { x } _ { j } \in \mathbb { X } \\
\mathbf { x } _ { i } \neq \mathbf { x } _ { j } } }\end{subarray}} D_{H}\left(\mathbf{x}_{i} \rightarrow \mathbf{x}_{j}\right) \\
& \cdot Q\left(\sqrt{\frac{1}{2 N_{0}}\left\|\mathbf{H U}\left(\mathbf{x}_{i}-\mathbf{x}_{j}\right)\right\|^{2}}\right) .
\end{aligned}
$$

The conjugate gradient of Eq. (31) with respect to $\mathbf{U}$ is given by

$$
\begin{aligned}
\nabla J e(\mathbf{U})= & \frac{-\mathbf{H}^{H} \mathbf{H U}}{4 \sqrt{\pi} N_{0}} \times \sum_{\substack{\mathbf{x}_{i} \in \mathbb{X} \\
\mathbf{x}_{j} \in \mathbb{X} \\
\mathbf{x}_{i} \neq \mathbf{x}_{j}}}\left\{D_{H}\left(\mathbf{x}_{i} \rightarrow \mathbf{x}_{j}\right)\right. \\
& \left.\cdot \varphi\left(\mathbf{x}_{i} \rightarrow \mathbf{x}_{j}\right) \cdot \exp \left(-\frac{\varepsilon}{4 N_{0}}\right)\left(\frac{\varepsilon}{4 N_{0}}\right)^{-\frac{1}{2}}\right\},
\end{aligned}
$$

where we have

$$
\begin{aligned}
& \varphi\left(\mathbf{x}_{i} \rightarrow \mathbf{x}_{j}\right)=\left(\mathbf{x}_{i}-\mathbf{x}_{j}\right)\left(\mathbf{x}_{i}-\mathbf{x}_{j}\right)^{H}=\mathbf{e}_{i j} \mathbf{e}_{i j}^{H}, \\
& \varepsilon=\left\|\mathbf{H U}\left(\mathbf{x}_{i}-\mathbf{x}_{j}\right)\right\|^{2} .
\end{aligned}
$$

It is worth noting that the TPC matrix $\mathbf{U}$ is a diagonal matrix, hence the final diagonal conjugate gradient matrix is constituted by the diagonal elements of $\nabla J e(\mathbf{U})$. The derivation of Eq. (32) is given in Appendix A. Given the conjugate gradient of Eq. (32), the problem of Eq. (30) can be solved iteratively by commencing the iterations from an appropriate initial point using the SCG algorithm of [36]. In order to have an initial diagonal TPC matrix $\mathbf{U}^{(1)} \in \mathbb{C}^{N_{t} \times N_{t}}$, we use the max- $d_{\text {min }}$ based TPC matrix solution for $\left(2 \times N_{r}\right)$-element $\mathrm{SM}$ systems and adopt the near-optimal max- $d_{\min }$ solution in Section IV for the other scenarios. ${ }^{3}$ Then, we optimize the TPC matrix with the aid of the SCG algorithm as follows:

\footnotetext{
${ }^{3}$ Moreover, we can also use the equally weighted diagonal matrix or other optimized TPC matrix of [33]-[35] as initial TPC matrix.
}

\section{Algorithm 2. The min-BER based TPC algorithm}

1) Initialization: Set a step size of $\mu>0$, a termination scalar of $\beta>0$ and a maximum number of iterations $N_{\text {all }}$; given the conjugate gradient of the initial diagonal TPC matrix $\mathbf{U}^{(1)}$ as $\tau(1)=\nabla J e\left(\mathbf{U}^{(1)}\right) \in \mathbb{C}^{N_{t} \times N_{t}}$, set $n=1$.

2) Loop: if $\left\|\nabla J e\left(\mathbf{U}^{(1)}\right)\right\|<\beta$ or $n>N_{\text {all }}$, goto Stop.

$$
\begin{aligned}
& \mathbf{U}^{(n+1)}=\mathbf{U}^{(n)}-\mu \tau(n) /\|\tau(n)\|, \\
& \alpha=P_{\mathrm{T}} / \operatorname{tr}\left(\mathbf{U}^{(n+1)}\left(\mathbf{U}^{(n+1)}\right)^{H}\right), \\
& \mathbf{U}^{(n+1)}=\sqrt{\alpha} \mathbf{U}^{(n+1)}, \\
& \varphi_{l}=\left\|\nabla J e\left(\mathbf{U}^{(n+1)}\right)\right\|^{2} /\left\|\nabla J e\left(\mathbf{U}^{(n)}\right)\right\|^{2}, \\
& \tau(n+1)=\varphi_{l} \tau(n)-\nabla J e\left(\mathbf{U}^{(n+1)}\right) .
\end{aligned}
$$

$n=n+1$, goto Loop.

3) Stop: $\mathbf{U}^{(n+1)}$ is the solution.

As shown in [36] and [37], the convergence of the SCG 436 algorithm is more rapid than that of the classic steepest gradient 437 algorithm. For the sake of avoiding convergence to a local opti- 438 mum, the values of $\varphi_{l}$ in SCG can be periodically reset either 439 to zero or to their negative counterparts [36]. ${ }^{4}$

\section{B. SCG Algorithm Based on the Simple Q-function Estimations}

In the SCG algorithm, the computational complexity is 442 dominated by the calculation of the conjugate gradient of 443 Eq. (32). To reduce this complexity, two simple upper 444 bounds of the Gaussian Q-function can be adopted. The 445 first well-known estimate is given by the Chernoff bound as 446 follows [39]

$$
Q(x) \leq \frac{1}{2} \exp \left(-\frac{x^{2}}{2}\right)
$$

Hence, the conjugate gradient of Eq. (32) with respect to $\mathbf{U}$ is 448 simplified to

$$
\begin{gathered}
\nabla J e_{C h e r}(\mathbf{U})=\frac{-\mathbf{H}^{H} \mathbf{H U}}{4 N_{0}} \times \sum_{\substack { \mathbf{x}_{i} \in \mathbb{X} \\
\begin{subarray}{c}{\mathbf{x}_{j} \in \mathbb{X} \\
\mathbf{x}_{i} \neq \mathbf{x}_{j}{ \mathbf { x } _ { i } \in \mathbb { X } \\
\begin{subarray} { c } { \mathbf { x } _ { j } \in \mathbb { X } \\
\mathbf { x } _ { i } \neq \mathbf { x } _ { j } } }\end{subarray}}\left\{D_{H}\left(\mathbf{x}_{i} \rightarrow \mathbf{x}_{j}\right)\right. \\
\left.\cdot \varphi\left(\mathbf{x}_{i} \rightarrow \mathbf{x}_{j}\right) \cdot \exp \left(-\frac{\varepsilon}{4 N_{0}}\right)\right\} .
\end{gathered}
$$

A more accurate approximation of the Q-function than the 450 Chernoff bound is formulated as a sum of weighted expo- 451 nentials. By considering only two components, the following 452 Chiani-bound has been proposed in [39]

$$
Q(x) \leq \frac{1}{12} \exp \left(-\frac{x^{2}}{2}\right)+\frac{1}{4} \exp \left(-\frac{2 x^{2}}{3}\right)
$$

\footnotetext{
${ }^{4}$ Further information about the SCG algorithm is available in [37].
} 


$$
\begin{aligned}
& \text { and the corresponding conjugate gradient } \nabla J e_{C h a i}(\mathbf{U}) \text { is } \\
& \nabla J e_{C h a i}(\mathbf{U})=\frac{-\mathbf{H}^{H} \mathbf{H U}}{4 N_{0}} \cdot \sum_{\substack{\mathbf{x}_{i} \in \mathbb{X} \\
\mathbf{x}_{\mathbf{x}_{j} \in \mathbb{X}}}}\left\{D_{H}\left(\mathbf{x}_{i} \rightarrow \mathbf{x}_{j}\right)\right. \\
& \left.\varphi\left(\mathbf{x}_{i} \rightarrow \mathbf{x}_{j}\right) \cdot\left[\frac{1}{6} \exp \left(-\frac{\varepsilon}{4 N_{0}}\right)+\frac{2}{3} \exp \left(-\frac{\varepsilon}{3 N_{0}}\right)\right]\right\} .
\end{aligned}
$$

Eqs. (40) and (42) provide two simple approximations of Eq. (32). It is worth noting that the transmit vectors of SM schemes are sparsely populated, since they have mostly zero values, hence the space of non-linear error vectors $\mathbf{e}_{i j}=\mathbf{x}_{i}$ $\mathbf{x}_{j}$ is small, as shown in Section III. For example, the number of non-linear error vectors $\mathbf{e}_{i j}$ for QPSK-modulated SM associated with $N_{t}=2$ is as low as six. In the SCG-based TPC optimization, we may only have to consider these non-linear error vectors and hence the computational complexity of the SCG algorithm can be further reduced.

\section{Min-BER Based TPC Matrix Design With Imperfect CSI}

In practical applications, pilot symbols are commonly used for estimating the MIMO channel, but naturally the estimated MIMO channel matrix is inevitably imperfect. Hence, the TPC design algorithm should give cognizance to the estimated MIMO channel matrix $\hat{\mathbf{H}}$, which is given by [42], [43]

$$
\hat{\mathbf{H}}=\mathbf{H}+\Delta \mathbf{H},
$$

where $\Delta \mathbf{H}$ is the channel estimation error matrix. Let us assume that $\Delta \mathbf{H}$ is uncorrelated with $\mathbf{H}$ and satisfies $\Delta \mathbf{H}^{H} \Delta \mathbf{H}=$ $\sigma_{e r r}^{2} \mathbf{I}_{N_{t}}$. Then, the corresponding gradient for SCG algorithm is computed as

$$
\begin{aligned}
& \nabla J e(\mathbf{U})=\frac{-\hat{\mathbf{H}}^{H} \hat{\mathbf{H}} \mathbf{U}}{4 \sqrt{\pi}} \cdot \sum_{\mathbf{x}_{i} \in \mathbb{X}} \frac{1}{\sigma_{e}^{2}} \sum_{\substack{\mathbf{x}_{j} \in \mathbb{X} \\
\mathbf{x}_{i} \neq \mathbf{x}_{j}}}\left\{D_{H}\left(\mathbf{x}_{i} \rightarrow \mathbf{x}_{j}\right)\right. \\
& \left.\varphi\left(\mathbf{x}_{i} \rightarrow \mathbf{x}_{j}\right) \exp \left(-\frac{\varepsilon \hat{\mathbf{H}}}{4 \sigma_{e}^{2}}\right)\left(\frac{\varepsilon_{\hat{\mathbf{H}}}}{4 \sigma_{e}^{2}}\right)^{-\frac{1}{2}}\right\}+\frac{\sigma_{e r r}^{2} \mathbf{U}}{4 \sqrt{\pi}} \sum_{\mathbf{x}_{i} \in \mathbb{X}} \frac{\mathbf{x}_{i} \mathbf{x}_{i}^{H}}{\sigma_{e}^{4}} \\
& \cdot \sum_{\mathbf{x}_{j} \in \mathbb{X}} D_{H}\left(\mathbf{x}_{i} \rightarrow \mathbf{x}_{j}\right) \cdot \exp \left(-\frac{\varepsilon_{\hat{\mathbf{H}}}}{4 \sigma_{e}^{2}}\right)\left(\frac{\varepsilon_{\hat{\mathbf{H}}}}{4 \sigma_{e}^{2}}\right)^{-\frac{1}{2}} \varepsilon,
\end{aligned}
$$

where we have

$$
\sigma_{e}^{2}=N_{0}+\left(\mathbf{U} \mathbf{x}_{i}\right)^{H} \Delta \mathbf{H}^{H} \Delta \mathbf{H} \mathbf{U} \mathbf{x}_{i}
$$

and

$$
\varepsilon_{\hat{\mathbf{H}}}=\left\|\hat{\mathbf{H}} \mathbf{U}\left(\mathbf{x}_{i}-\mathbf{x}_{j}\right)\right\|^{2} .
$$

The derivation details of Eq. (44) are given in Appendix. As shown in Eq. (44), the resultant gradient carefully takes the channel estimation errors into account, when constructing the diagonal TPC matrix. Hence, the BER performance becomes resilient to CSI errors.
TABLE I

COMPUtATIONAL COMPLEXITy IMPOSED By THE GRADIENT $\nabla J e(\mathbf{U})$

\begin{tabular}{|l|l|}
\hline & Complexity imposed \\
\hline $\mathbf{H}^{H} \mathbf{H}$ & $O_{1}=N_{t}^{2}\left(2 N_{r}-1\right)$ \\
\hline$\bullet \times \mathbf{U}$ & $O_{2}=N_{t} N_{r}$ \\
\hline$\varphi\left(\mathbf{x}_{i} \rightarrow \mathbf{x}_{j}\right)$ & $O_{3}=N_{t}\left(\begin{array}{l}2 \\
M\end{array}\right)+4\left(\begin{array}{c|}2 \\
N_{t}\end{array}\right) M^{2}$ \\
\hline$\varepsilon=\left\|\mathbf{H U}\left(\mathbf{x}_{i}-\mathbf{x}_{j}\right)\right\|^{2}$ & $O_{4}=N_{t}\left(\begin{array}{l}2 \\
M\end{array}\right)\left(2 N_{r}-1\right)+4\left(\begin{array}{c}2 \\
N_{t}\end{array}\right) M^{2}\left(2 N_{r}-1\right)$ \\
\hline
\end{tabular}

\section{Complexity Analysis of the Proposed TPC ALGORITHMS}

In this section, we provide complexity evaluations of the 484 proposed max $-d_{\min }$ based TPC and the min-BER based TPC 485 algorithms, where only the multiplications of complex numbers 486 are considered.

Based on a similar analysis method to that of [33], for the 488 case of $N_{t}=2$, the closed-form solution of the max- $d_{\min }$ based 489 TPC can be found by using the Algorithm 1, which imposes a 490 complexity of

$$
\begin{aligned}
O_{\max -d_{\min }=} & \underbrace{4\left(2 N_{r}-1\right)}_{\text {calculate } \mathbf{H}^{H} \mathbf{H}}+\underbrace{15 M(M-1) / 2}_{\text {calculate optimal phase }} \\
& +\underbrace{(2 M+1)(M+7)}_{\text {calculate optimal PA }} .
\end{aligned}
$$

Moreover, for the case of $N_{t}>2$, an iterative max- $d_{\min }$ based 492 TPC can be adopted and the associated complexity (similar to 493 Eq. (22) of [33]) is

$$
\begin{aligned}
O_{\max }-d_{\min }= & \underbrace{4\left(2 N_{r}-1\right)}_{\text {calculate } \mathbf{H}^{H} \mathbf{H}}+\underbrace{\left(\begin{array}{c}
2 \\
N_{t}
\end{array}\right)(2 M-1)}_{\text {intial } d_{\text {min }}}+\cdots \\
n_{\mathrm{TPC}} & {[\underbrace{15 M(M-1) / 2}_{\text {calculate optimal phase }}+\underbrace{(2 M+1)(M+7)}_{\text {calculate optimal PA }}} \\
& +\underbrace{2\left(N_{t}-2\right)(2 M-1)}_{\text {optimized } d_{\text {min }}}],
\end{aligned}
$$

where $n_{\text {TPC }}$ is the number of iterations in the max $-d_{\min }$ based 495 TPC algorithm, which is varied according to the channel matrix. 496 In our simulations, we found that the average value of $n_{\mathrm{TPC}}$ is 497 approximated to 5 .

The complexity of the proposed min-BER based TPC algo- 499 rithm can be estimated by considering: (a) the computational 500 complexity of the SCG solution process in each iteration 501 and (b) the number of iterations $n_{\mathrm{SCG}}$ required for approach- 502 ing convergence. The first term can be estimated based on 503 Eqs. (34)-(38). In Table I, we characterize the computational 504 complexity imposed by the gradient $\nabla J e(\mathbf{U})$, where the sparse 505 structure of the SM symbols $\mathbf{x}_{i}, \mathbf{x}_{j}$ and of the diagonal TPC 506 matrix $\mathbf{U}$ are exploited. To be specific, the error vectors $\mathbf{e}_{i j}=507$ $\mathbf{x}_{i}-\mathbf{x}_{j}, i \neq j$ can be classified into two sets: $N_{t}\left(\begin{array}{c}2 \\ M\end{array}\right)$ vectors hav- 508 ing a single non-zero element and $\left(\begin{array}{c}2 \\ N_{t}\end{array}\right) M^{2}$ vectors having two 509 non-zero elements. They have different complexity for the cal- 510 culation of $\varphi\left(\mathbf{x}_{i} \rightarrow \mathbf{x}_{j}\right)$ and $\varepsilon$, as shown in Table I. Note that 511 
(a) $2 \times 2$ QPSK
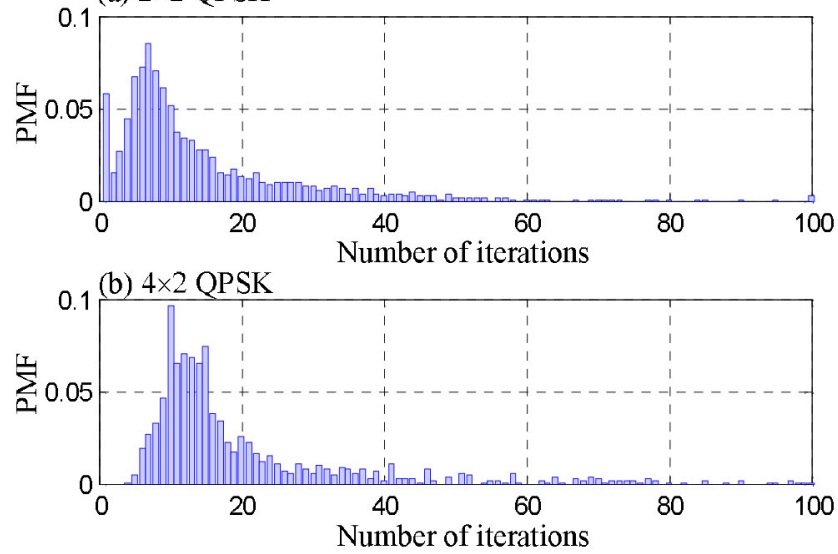

Fig. 3. Convergence behavior of the proposed min-BER based TPC in SM systems with QPSK modulation in i.i.d Rayleigh fading channels.

the bit-to-symbol mapping rule can be designed off-line, hence the complexity of $D_{H}\left(\mathbf{x}_{i} \rightarrow \mathbf{x}_{j}\right)$ is not considered in the calculation of $\nabla J e(\mathbf{U})$. Based on Table I and the SCG algorithm of Eqs. (34)-(38), the associated complexity of the proposed min-BER based TPC algorithm is approximately

$$
\begin{gathered}
O_{\min -\mathrm{BER}}=O_{1}+O_{3}+n_{\mathrm{SCG}}[5\left(O_{2}+O_{4}\right)+\underbrace{N_{t}^{2}+N_{t}}_{\mathrm{Eq} .34} \\
+\cdots \underbrace{N_{t}^{2}-N_{t}+1}_{\text {Eq. } 35}+\underbrace{N_{t}}_{\text {Eq. } 36}+\underbrace{N_{t}^{2}-N_{t}+1}_{\text {Eq. } 37}+\underbrace{2 N_{t}}_{\text {Eq. } 38}] .
\end{gathered}
$$

Moreover, similar to [30], in Fig. 3 we have portrayed the probability mass function (PMF) of the numbers of iterations for the min-BER based TPC algorithm in the QPSK-modulated $(2 \times 2)$ and $(4 \times 2)$ SM schemes. In the simulations, the threshold of SCG is given by $\beta=10^{-5}$ and 25000 trails are considered to show the statistics of convergence. In Fig. 3 (a) and (b), more than $90 \%$ and $85 \%$ of the trials converged within 30 iterations. This is due to the rapid convergence of the SCG algorithm, as also verified in [37]. Note that although the approximation method of Section IV-B can reduce the complexity of calculating $\nabla \mathrm{Je}(\mathbf{U})$, i.e. the complexity terms $\mathrm{O}_{3}$ and $\mathrm{O}_{4}$ in Table I, it has the same complexity order as (49). We will provide more detailed comparisons and discussions about the complexity issue in Section VI-C.

\section{Simulation Results}

In this section, we provide simulation results (the distance $d_{\min }$ and the BER performance) for characterizing the max$d_{\min }$ based TPC aided SM and the min-BER based TPC aided SM schemes for transmission over frequency-flat fading channels. For comparison, these performance results are compared to various adaptive SM schemes, such as the ASM arrangements of [21], the maximum minimum distance (MMD) aided SM schemes of [30], the PA-based SM schemes of [33], the
TPC star-QAM SM schemes of [31], and the PRP aided SM 540 schemes of [29] and [34], [35]. $\quad 541$

In the min-BER based TPC scheme, the step size $\mu$ is deter- 542 mined by Monte Carlo simulation methods, as suggested in 543 [36] and we set $\mu=0.01$, so that we achieve a rapid conver- 544 gence, while maintaining excellent BER results. Moreover, for 545 the BPSK case, we do not consider the ASM scheme because 546 'no-transmission' is assigned to one of the TAs and hence this 547 TA is inactive [33].

\section{A. $d_{\min }$ Performance for Different SM Schemes}

In Fig. 4, we compare the complementary cumulative distri- 550 bution functions (CCDF) of the distance $d_{\min }$ recorded for both 551 for conventional SM and for the link adaptive SM schemes in 552 $(2 \times 1)$ MIMO channels under different throughputs. First, we 553 note that these adaptive SM schemes are capable of beneficially 554 increasing the distance $d_{\min }$. As formally shown in Section III, 555 we observe in Fig. 4 that the proposed max- $d_{\min }$ based TPC 556 aided SM achieves the highest distance $d_{\min }$ compared to other 557 link-adaptive SM schemes. Furthermore, we note that the min- 558 BER based TPC schemes achieve lower $d_{\min }$ than the max- $d_{\min } \quad 559$ based TPC schemes, and yet we will see in Figs. 5-7 that the 560 min-BER based TPC outperforms the max- $d_{\min }$ based TPC in 561 terms of its BER.

\section{B. BER Comparisons of Different SM Schemes}

In Fig. 5, we compare the BER performance of various SM 564 systems for $L=2$ bits/symbol in $(2 \times 1)$ - and $(2 \times 2)$-element 565 MIMO channels. We can see that the proposed min-BER based 566 schemes provides gains of about $6 \mathrm{~dB}$ and $4 \mathrm{~dB}$ at the BER of 567 $10^{-3}$ over the conventional SM schemes. We also confirm that 568 the min-BER based schemes outperform the ASM of [21], the 569 max $-d_{\min }$ based PA aided SM of [33] and the $\max -d_{\min }$ based 570 TPC aided SM proposed.

Note that, as shown in Fig. 4, although the optimal max- 572 $d_{\min }$ based TPC aided SM is capable of achieving a higher 573 distance $d_{\min }$ than the other adaptive SM schemes, it does 574 not achieve a BER performance improvement over them. To 575 expound a little further, in Fig. 5, when the proposed max- $d_{\min } 576$ based TPC-aided SM is compared to its special case, namely 577 to the max- $d_{\min }$ based PA aided SM, we find that an increase 578 of the distance $d_{\min }$ by TPC does not achieve any further BER 579 improvement. Observe in Fig. 5 that at high SNRs, the max- 580 $d_{\min }$ based TPC aided SM may even perform worse than the 581 $\max -d_{\min }$ based PA aided SM. This is mainly due to the fact that 582 the maximum of $d_{\min }$ does not necessarily minimize the PEP 583 bound of Eq. (29), which depends on all the received distances. 584

To be specific, the reason for the trends of Fig. 5 is that the 585 max- $d_{\min }$ based TPC may achieve a lower Euclidean distance 586 between the non-adjacent received constellation points than 587 that of the PA schemes. Hence, based on the Q-function aided 588 PEP upper bound of Eq. (29), which depends on all legitimate 589 received distances $d_{i j}(\mathbf{H})=\left\|\mathbf{H U}\left(\mathbf{x}_{i}-\mathbf{x}_{j}\right)\right\|(i \neq j)$, the max- 590 $d_{\min }$ based TPC fails to achieve the best BER performance. For 591 example, let us consider the $(2 \times 1)$ SM scheme using BPSK. 592 As shown in Section III, we only have four different distances 593 

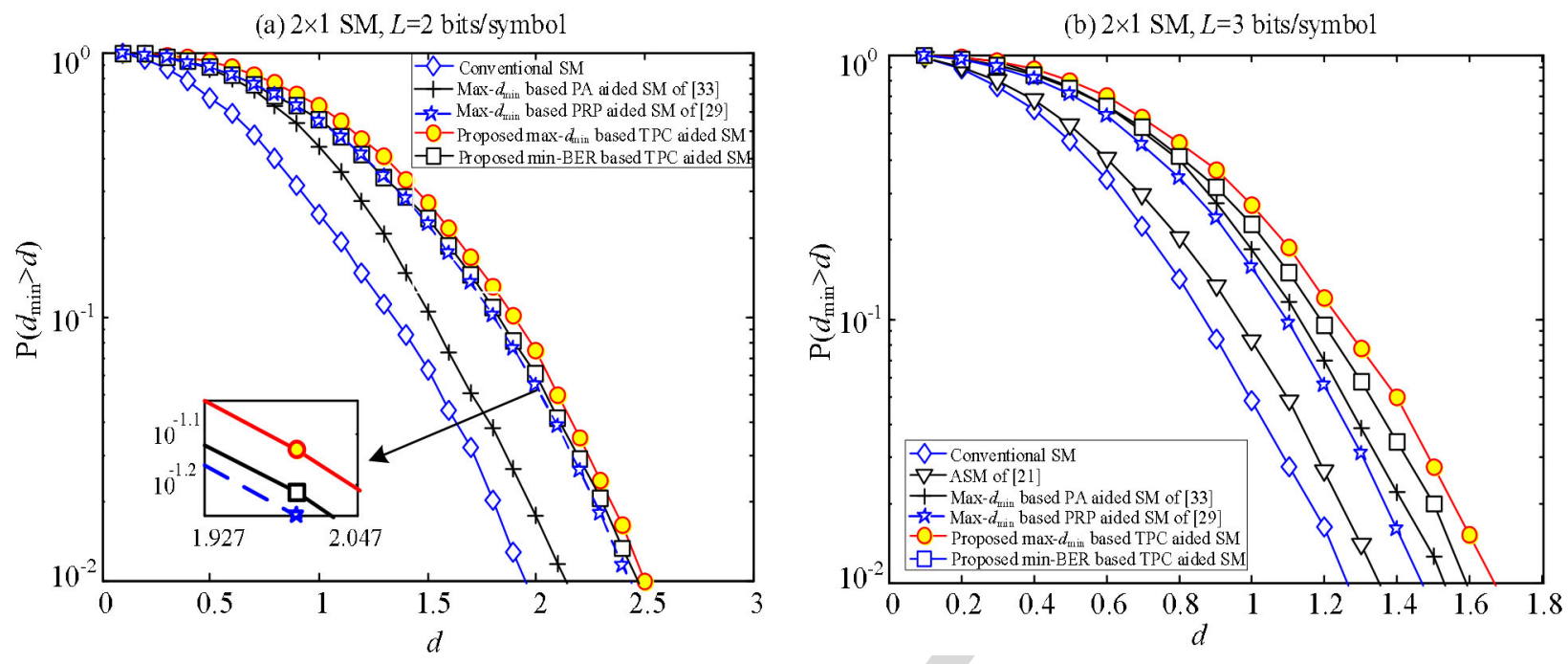

Fig. 4. The CCDF of the minimum distance $d_{\min }$ of conventional SM and of various link-adaptation aided SM schemes in $(2 \times 1)$-element MIMO channels.

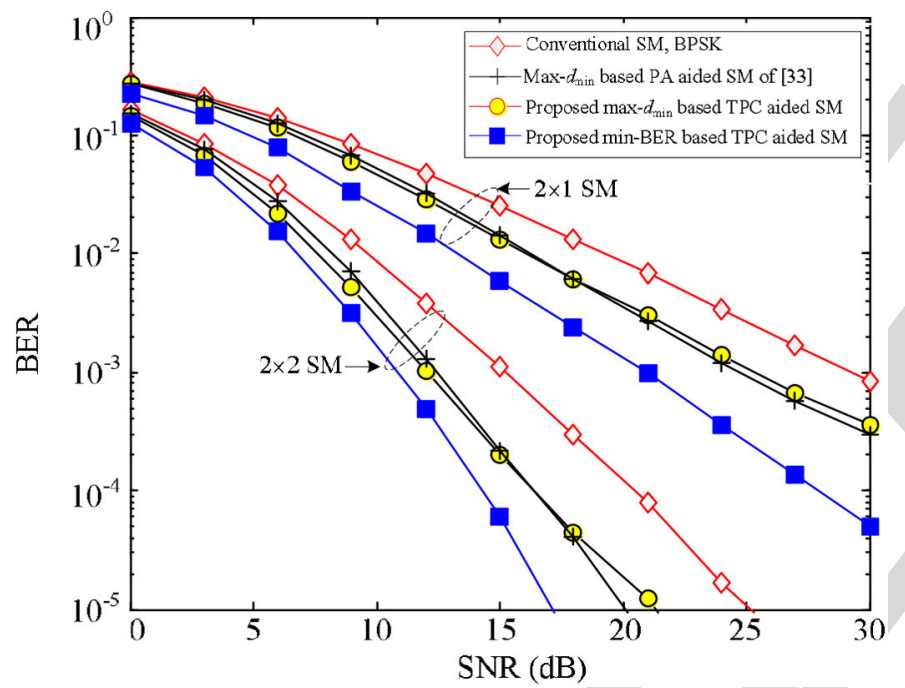

Fig. 5. BER comparison at $L=2$ bits/symbol for the conventional SM, for the max- $d_{\text {min }}$ based TPC aided SM and for the min-BER based TPC aided SM.

at the receiver, namely $d_{1}, d_{2}, d_{3}$ and $d_{4}$. Assuming that the channel matrix is $\mathbf{H}=[0.056-0.069 i, 0.414+1.267 i]$ and the $\mathrm{SNR}$ is $25 \mathrm{~dB}$, the resultant distance set for the $\max -d_{\min }$ based TPC-aided SM is

$\mathbb{D}_{T P C}=\left\{d_{1}=0.251, d_{2}=0.434, d_{3}=0.251, d_{4}=0.251\right\}$,

while the corresponding distance set for the $\max -d_{\min }$ based PA aided SM is

$$
\mathbb{D}_{P A}=\left\{d_{1}=0.248, d_{2}=0.718, d_{3}=0.476, d_{4}=0.248\right\} .
$$

Then, the BER results of Eq. (29) calculated for the TPC-aided SM and the PA-aided SM schemes are $P_{e}(\mathbf{H})=0.7 \times 10^{-3}$ and $P_{e}(\mathbf{H})=0.5 \times 10^{-3}$, respectively. This result confirms that although the max $-d_{\min }$ based TPC algorithm achieves the highest distance of $d_{\min }=0.251$, while the $\max -d_{\min }$ based PA algorithm has $d_{\min }=0.248$, the former has a worse PEP performance due to its lower values of $d_{2}$ and $d_{3}$. This result is consistent with the result seen in Fig. 5.

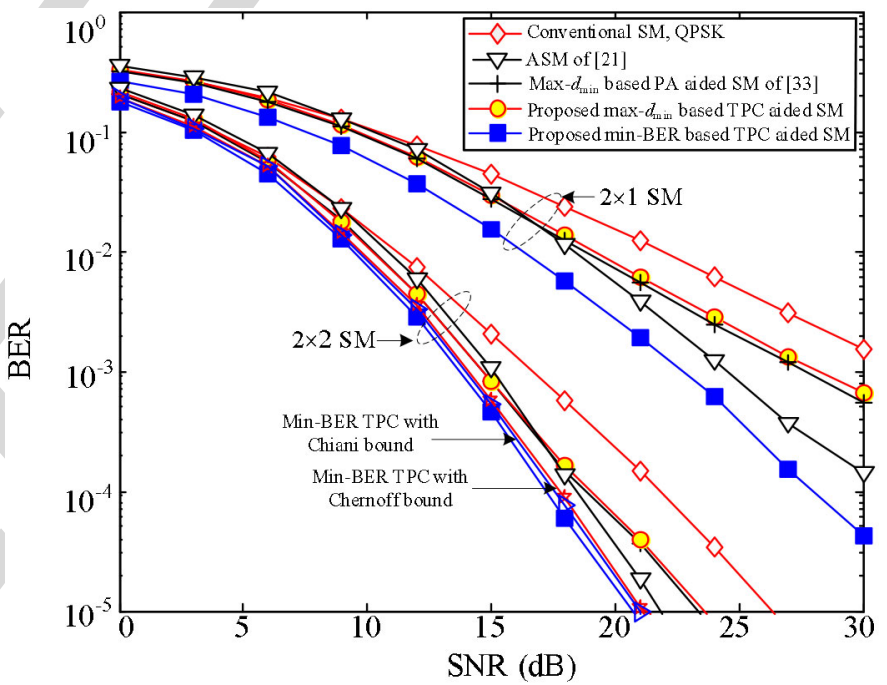

Fig. 6. BER comparison at $L=3$ bits/symbol for various SM schemes. Here, the Q-function estimates of Section IV-B are only considered for the $(2 \times 2)$ element MIMO channels.

The above-mentioned trends of these proposed TPC algo- 608 rithms are also visible in Fig. 6, where the throughput is $L=3609$ bits/symbol. It is shown in Fig. 6 that the proposed min-BER 610 based TPC outperforms both ASM of [21] and the max- $d_{\min } 611$ based PA of [33]. Moreover, in Fig. 6, we demonstrate that the 612 approximate Chernoff-based and Chiani-based optimizations 613 perform almost the same as the exact Q-function based scheme. 614 This is because these approximations do not change the direc- 615 tion of the gradient. We have also simulated the Chernoff-based 616 and Chiani-based optimizations for the other MIMO setups 617 considered, and obtained similar results, as evidenced by Fig. 6. 618 Since the resultant curves approximately overlap with the opti- 619 mal one, for clarity, these results are not included in other 620 figures.

Due to the advantage of the proposed min-BER based TPC, 622 in Fig. 7 we further investigate its performance for a higher 623 number of TAs and modulation order. All the schemes are 624 


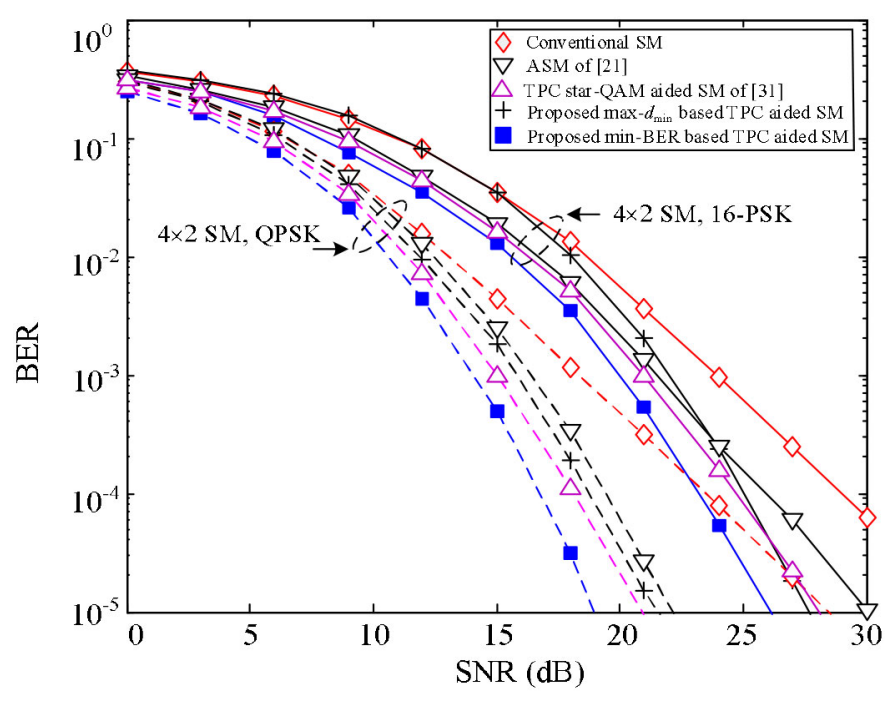

Fig. 7. BER comparison of the proposed min-BER-based TPC-aided SM schemes over the TPC star-QAM aided SM schemes.

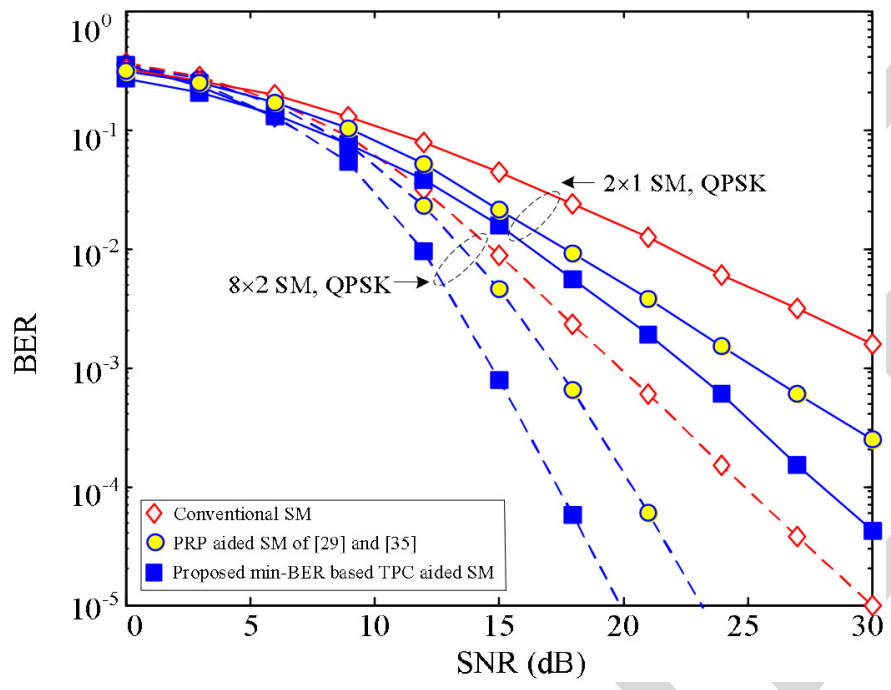

Fig. 8. BER comparison of the proposed min-BER-based TPC-aided SM schemes over the PRP-aided SM schemes. assumed to have $N_{t}=4, N_{r}=2$ and the throughputs are $L=4$ and $L=6$ bits/symbol. In Fig. 7, the proposed schemes are also compared to the TPC star-QAM aided SM schemes of [31], which utilize a quantized search for optimizing the diagonal TPC matrix. In our simulation, the number of quantization levels for both amplitude and phase in TPC of [31] is 6. Observe in Fig. 7 that the proposed min-BER based TPC schemes achieve the best BER performance. The performance gain of the proposed scheme over the TPC star-QAM aided SM scheme is seen to be about $2.6 \mathrm{~dB}$ at $\mathrm{BER}=10^{-5}$ for 4 bits/symbol transmissions in Fig. 7. This is due to the fact the TPC star-QAM based scheme of [31] also only optimizes a single received distance $d_{\text {min }}$, which may limit the attainable BER performance.

In Fig. 8, we compared the proposed min-BER based TPC schemes to the max- $d_{\min }$ based PRP schemes of [29] and [35]. We observe in Fig. 8 that the proposed schemes outperform the PRP-aided schemes. To be specific, as seen in Fig. 8 the

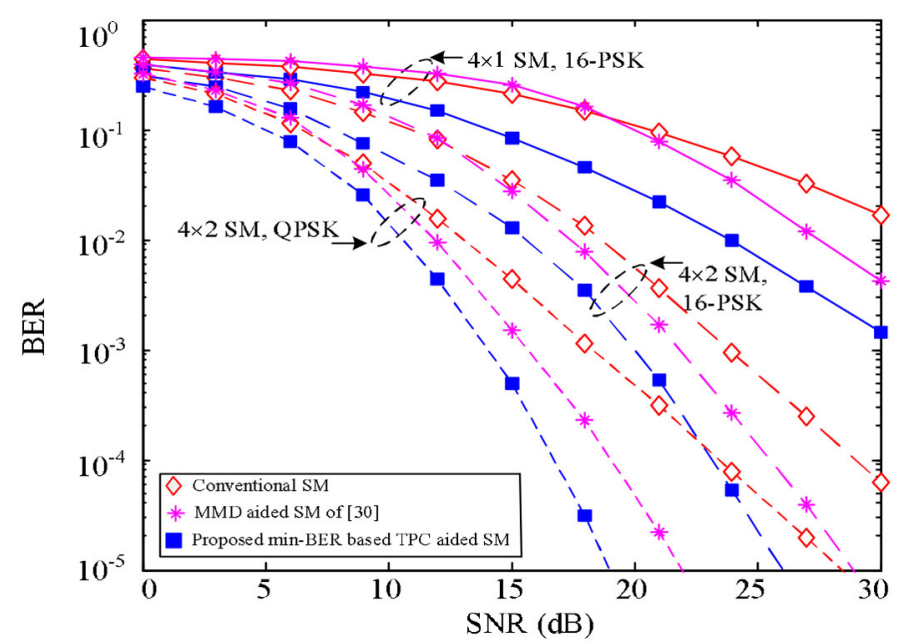

Fig. 9. BER comparison of the proposed min-BER based TPC aided SM schemes over the MMD aided SM schemes.

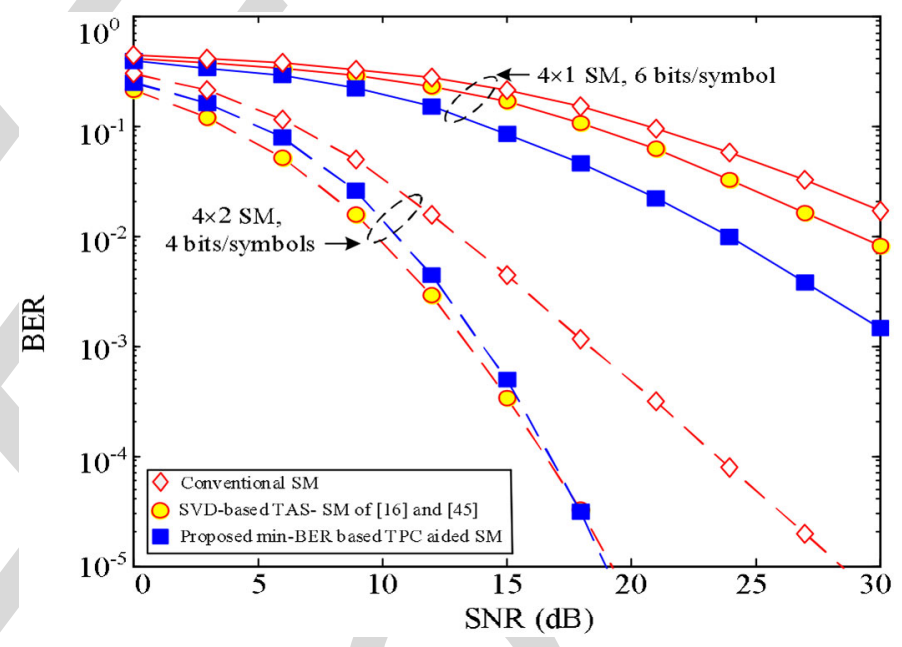

Fig. 10. BER performance of the proposed min-BER-based TPC-aided SM schemes and the SVD-based TAS-SM schemes.

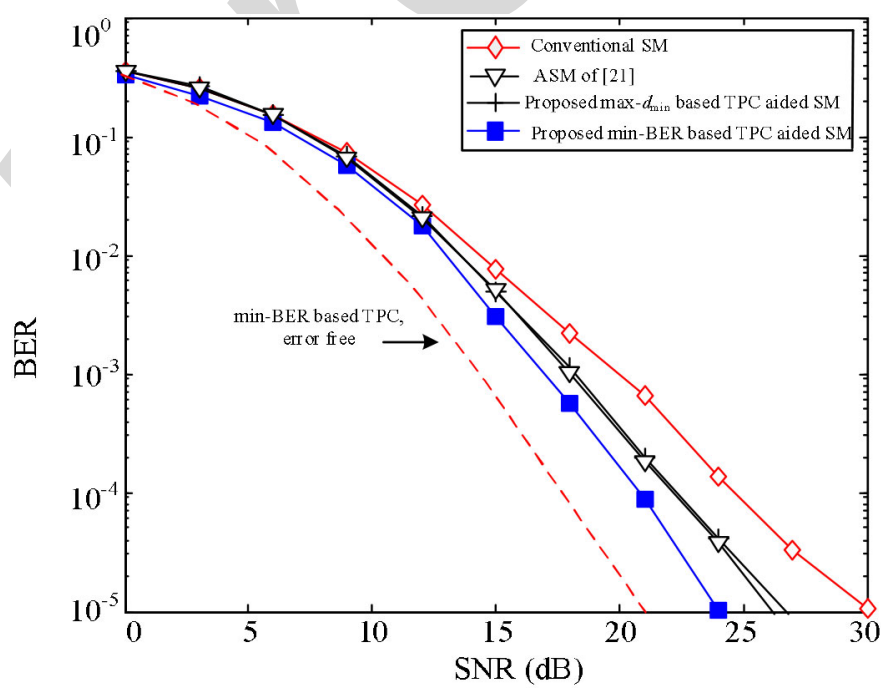

Fig. 11. BER performance of different adaptive SM schemes in the presence of CSI errors. Here, the error variance is $\sigma_{\text {err }}^{2}=1 / r$, where $r$ is the average SNR at each receiver antenna. 
TABLE II

COMPLEXITy ORders For DifFERENT TPC DESigns. THE EXAMPLES ARE With PARAMETERS $n_{\mathrm{SCG}}=30, n_{\mathrm{PA}}=2, n_{\mathrm{PRP}}=3, n_{\mathrm{TPC}}=5, L_{1}=6 \mathrm{AND} L_{2}=6$

\begin{tabular}{|l|l|l|l|}
\hline Designs & Complexity order & $\begin{array}{l}\text { Configuration 1 } \\
(4 \times 2, \mathrm{QPSK})\end{array}$ & $\begin{array}{l}\text { Configuration 2 } \\
(4 \times 2 \text { 16PSK })\end{array}$ \\
\hline ASM of [21] & $\mathcal{O}\left(N_{t}^{2} M^{2}\right)+\mathcal{O}\left(N_{t}^{2} N_{r}\right)$ & 320 & 4160 \\
\hline Max- $d_{\min }$ based PRP of [34] & $\mathcal{O}\left(N_{t}^{2} M\right)+\mathcal{O}\left(n_{\mathrm{PRP}}\left(M^{2}+N_{t} M\right)\right)$ & 160 & 1216 \\
\hline Max- $d_{\min }$ based PA of [33] & $\mathcal{O}\left(N_{t}^{2} M\right)+\mathcal{O}\left(n_{\mathrm{PA}}\left(M^{2}+N_{t} M\right)\right)$ & 128 & 896 \\
\hline Diagonal TPC method of [32] & $\mathcal{O}\left(L_{1} L_{2} N_{t}^{2} M^{2} N_{r}\right)$ & 18432 & 294912 \\
\hline MMD based TPC of [30] & $\mathcal{O}\left(N_{t}^{2} M^{2} N_{r}\right)+\mathcal{O}\left(N_{t}^{4} M^{4}\right)$ & 66048 & 16785408 \\
\hline Proposed max- $d_{\min }$ based TPC & $\mathcal{O}\left(N_{t}^{2} M\right)+\mathcal{O}\left(n_{\mathrm{TPC}}\left(M^{2}+N_{t} M\right)\right)$ & 224 & 1856 \\
\hline Proposed min-BER based TPC & $\mathcal{O}\left(n_{\mathrm{SCG}} N_{t}^{2} M^{2} N_{r}\right)$ & 15360 & 245760 \\
\hline
\end{tabular}

proposed TPC scheme provides about $3.2 \mathrm{~dB}$ gain over the PRP scheme at BER $=10^{-5}$ for $(8 \times 2)$-element MIMO channels at a throughput of 5 bits/symbol. This benefit is due to the following two reasons: (1) the PRP schemes only adapt the phases of the SM symbols and hence the degrees of freedom utilized for TPC design are limited [35]; (2) similar to the methods of [31], [33], they are designed based on the max- $d_{\min }$ principle and hence may provide suboptimal BER.

In Fig. 9, the proposed min-BER based TPC schemes are compared to the MMD-aided SM schemes of [30]. Observe in Fig. 9 that the proposed TPC scheme provides an SNR gain of about $3 \mathrm{~dB}$ over the MMD-aided scheme at the BER of $10^{-5}$ for the $(4 \times 2)$ MIMO channels considered. Similar to the results in [30], the MMD-based TPC schemes provide minor performance improvements or even degrade the performance in low-SNR regimes. This is because the MMD criterion based TPC design may be ineffective in low SNR regimes, as discussed in [30].

Moreover, in Fig. 10, the proposed min-BER based TPC schemes are compared to the TAS-based SM schemes [16], [17], [44], [45] under different throughputs. In Fig. 10, the singular value decomposition (SVD)-based TAS algorithm of [16], [44], [45] is utilized due to its low-complexity and attractive performance. The number of TAs is $N_{t}=4$ and 2 out of $N_{t}=4$ TAs are selected by the TAS algorithm. Without loss of generality, we consider a PSK signal constellation diagram. As shown in Fig. 10, the TAS and the TPC schemes exhibit different BER advantages for different system setups. Specifically, the proposed TPC scheme outperforms the TAS scheme for $(4 \times 1)$ MIMO channels having a throughput of 6 bits/symbol, while they achieve a similar BER performance for $(4 \times 2)$ MIMO associated with 4 bits/symbol. This is not surprising, since the TAS and the TPC algorithms rely on different transmit parameters for the sake of achieving BER improvements. Note that TPC can be added on top of TAS to further improve performance. Hence they are complementary rather than competitive. It has been shown in [24], [30] that the joint design of TPC and TAS can further improve the system performance.

Fig. 11 shows the BER performance of various SM schemes in the presence of Gaussian-distributed CSI errors obeying eN $\left(0, \sigma_{e r r}^{2}\right)$ [42], [43] for $(2 \times 2)$ MIMO channels and $L=3$ bits/symbol. For the sake of simplification and clarity, we only consider the ASM and PA-aided SM schemes as benchmarks. In this paper, the variable $\sigma_{e r r}^{2}$, i.e. the value of the estimation error is adjusted according to the SNR. To be specific, $\sigma_{e r r}^{2}=1 / r$ is 687 adopted, where $r$ is the average SNR at each receiver antenna. 688 As expected, the BER performance of all SM schemes degrades 689 upon imposing CSI estimation errors. However, Fig. 11 shows 690 that the performance degradation of the proposed min-BER 691 based TPC-aided SM is lower than that of the other schemes 692 due to the fact that its BER upper bound was optimized bearing 693 in mind the CSI error by using the SCG algorithm.

\section{Complexity Comparison for Different TPC Designs}

In Table II, the complexity orders of different TPC designs 696 are compared. Specifically, in the randomly-selected diagonal 697 TPC method of [31], [32], the quantization levels of ampli- 698 tude and phase are $L_{1}$ and $L_{2}$, respectively. Its complexity 699 order is provided in [30]. Moreover, the complexity orders of 700 the max $-d_{\min }$ based PRP and of the $\max -d_{\min }$ based PA algo- 701 rithms can be found in [34] and [33], where their iteration 702 numbers are $n_{\mathrm{PA}}$ and $n_{\mathrm{PRP}}$, respectively. In Table II, we also 703 provide the approximate quantified complexity for some spe- 704 cific configurations, where the QPSK- and 16PSK-modulated 705 $4 \times 2$ SM schemes are considered. The number of iterations for 706 the proposed SCG method is set to be $n_{\mathrm{SCG}}=30$ due to it fast 707 convergence.

As shown in Table II, the proposed $\max -d_{\min }$ based TPC 709 has a similar complexity order to that of the max- $d_{\min }$ based 710 PRP of [34] and to that of the PA of [33], while exhibiting 711 a lower complexity than the proposed min-BER design, since 712 these $\max -d_{\min }$ based designs only have to optimize a single 713 distance $d_{\min }$. However, as shown in our simulation results of 714 Figs 5-7 these max $-d_{\min }$ based designs suffer from a BER per- 715 formance loss. The MMD-based TPC of [30] is a generalized 716 max $-d_{\text {min }}$ based TPC, which has to optimize $N_{t} M$ TPC weights 717 for all legitimate SM symbols rather than relying on a diago- 718 nal TPC matrix having only $N_{t}$ non-zero elements. Hence, the 719 MMD-based TPC imposes a higher complexity than the pro- 720 posed max $-d_{\min }$ and min-BER based TPC algorithms, as shown 721 in Table II. For example, in configuration 2, the complexity of 722 the proposed min-BER based design approximately achieves 68723 times smaller than that of the MMD-based design.

Moreover, the diagonal TPC method of [32] requires an 725 exhaustive search over a set of $L_{1} L_{2}$ candidates, hence a higher 726 complexity is imposed compared to the proposed min-BER 727 based TPC for a high number of quantization levels. By tak- 728 ing into account both the BER versus complexity trends, we 729 


$$
\varepsilon_{\hat{\mathbf{H}}}=\left\|\hat{\mathbf{H}} \mathbf{U}\left(\mathbf{x}_{i}-\mathbf{x}_{j}\right)\right\|^{2} .
$$

761 Note that $\sigma_{e}^{2}$ and $\varepsilon_{\hat{\mathbf{H}}}$ are functions of the TPC matrix $\mathbf{U}$. Then 762

conclude that the proposed min-BER based TPC provides an improved BER performance at a modest complexity cost. It should be noted that the extra complexity is imposed by the calculation of the gradient $\nabla J e(\mathbf{U})$ and by the convex problem solution algorithm, which may be further reduced by exploiting the spatial-domain sparsity of SM symbols and with the aid of reduced-complexity solution techniques. This issue will be investigated in our future studies.

\section{CONCLUSIONS}

We have investigated two types of diagonal TPC design algorithms. For the max- $d_{\text {min }}$ based TPC algorithm, closed-form solutions were derived for the case of two TAs and suboptimal solutions were achieved by using iterative method. For the min-BER based TPC algorithm, an iterative SCG algorithm was proposed for finding the specific TPC matrix solution. Finally, the proposed min-BER based TPC algorithm was further enhanced by taking into account the effects of imperfect CSI. It is shown from simulation results that the proposed max$d_{\text {min }}$ based TPC algorithm is optimal in terms of the minimum received distance, while the proposed min-BER based TPC algorithm is optimal in terms of the BER. Our further work will be focused on the integration of space-time coding, channel coding and TAS techniques into the proposed schemes.

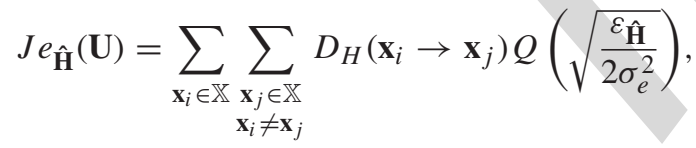

$$
\sigma_{e}^{2}=N_{0}+\sigma_{e r r}^{2}\left(\mathbf{U} \mathbf{x}_{i}\right)^{H} \mathbf{U} \mathbf{x}_{i}
$$

the gradient of $J e_{\hat{\mathbf{H}}}(\mathbf{U})$ can be expressed as:

$$
\begin{aligned}
\nabla J e_{\hat{\mathbf{H}}}(\mathbf{U}) & =\frac{\partial J e_{\hat{\mathbf{H}}}(\mathbf{U})}{\partial \mathbf{U}^{H}} \\
& =\sum_{\mathbf{x}_{i} \in \mathbb{X}} \sum_{\substack{\mathbf{x}_{j} \in \mathbb{X} \\
\mathbf{x}_{i} \neq \mathbf{x}_{j}}} D_{H}\left(\mathbf{x}_{i} \rightarrow \mathbf{x}_{j}\right) \frac{\partial Q\left(\sqrt{\frac{\varepsilon \hat{\mathbf{H}}}{2 \sigma_{e}^{2}}}\right)}{\partial \mathbf{U}^{H}} .
\end{aligned}
$$

Here, $\partial Q\left(\sqrt{\frac{\varepsilon_{\hat{\mathbf{H}}}}{2 \sigma_{e}^{2}}}\right) / \partial \mathbf{U}^{H}$ of Eq. (53) can be expressed as

$$
\begin{aligned}
& \frac{\partial Q\left(\sqrt{\frac{\varepsilon_{\mathbf{H}}}{2 \sigma_{e}^{2}}}\right)}{\partial \mathbf{U}^{H}}=-\frac{1}{\sqrt{\pi}} e^{-\frac{\varepsilon_{\mathbf{H}}}{4 \sigma_{e}^{2}}}\left(\frac{\varepsilon_{\hat{\mathbf{H}}}}{4 \sigma_{e}^{2}}\right)^{-\frac{1}{2}} \frac{\partial\left(\sqrt{\frac{\varepsilon_{\hat{\mathbf{H}}}}{4 \sigma_{e}^{2}}}\right)}{\partial \mathbf{U}^{H}} \\
& =-\frac{1}{\sqrt{\pi}} e^{-\frac{\varepsilon_{\hat{\mathbf{H}}}}{4 \sigma_{e}^{2}}}\left(\frac{\varepsilon_{\hat{\mathbf{H}}}}{4 \sigma_{e}^{2}}\right)^{-\frac{1}{2}}\left\{\left(\frac{\partial\left(\varepsilon_{\hat{\mathbf{H}}}\right)}{\partial \mathbf{U}^{H}} \frac{1}{4 \sigma_{e}^{2}}\right)+\left(\frac{\partial\left(\frac{1}{4 \sigma_{e}^{2}}\right)}{\partial \mathbf{U}^{H}} \varepsilon_{\hat{\mathbf{H}}}\right)\right\},
\end{aligned}
$$

where we have the relationship of

$$
\begin{aligned}
\frac{\partial\left(\varepsilon_{\hat{\mathbf{H}}}\right)}{\partial \mathbf{U}^{H}} & =\hat{\mathbf{H}}^{H} \hat{\mathbf{H}} \mathbf{U} \varphi\left(\mathbf{x}_{i} \rightarrow \mathbf{x}_{j}\right), \\
\frac{\partial\left(\frac{1}{4 \sigma_{e}^{2}}\right)}{\partial \mathbf{U}^{H}} & =-\frac{\sigma_{e r r}^{2} \mathbf{U} \mathbf{x}_{i} \mathbf{x}_{i}^{H}}{4 \sigma_{e}^{4}} .
\end{aligned}
$$

Based on Eq. (56), we can arrive at the gradient matrix of 765 Eq. (44). Moreover, assuming that the CSI is perfectly known, 766 we have $\Delta \mathbf{H}=0, \hat{\mathbf{H}}=\mathbf{H}$ and $\varepsilon_{\hat{\mathbf{H}}}=\varepsilon=\left\|\mathbf{H U}\left(\mathbf{x}_{i}-\mathbf{x}_{j}\right)\right\|^{2} .767$ Then, the gradient matrix of Eq. (32) is readily obtained.

\section{REFERENCES}

[1] R. Y. Mesleh, H. Haas, S. Sinanovic, C. W. Ahn, and S. Yun, "Spatial 770 modulation," IEEE Trans. Veh. Technol., vol. 57, no. 4, pp. 2228-2241, 771 Jul. 2008.

[2] M. Di Renzo, H. Haas, A. Ghrayeb, S. Sugiura, and L. Hanzo, "Spatial 773 modulation for generalized MIMO: Challenges, opportunities and imple- 774 mentation," Proc. IEEE, vol. 102, no. 1, pp. 56-103, Jan. 2014.

[3] S. Sugiura, S. Chen, and L. Hanzo, "A universal space-time architecture 776 for multiple-antenna aided systems," IEEE Commun. Surveys \& Tuts., 777 vol. 14, no. 2, pp. 401-420, May 2012.

[4] E. Başar, Ü. Aygölü, E. Panayırc1, and H. V. Poor, "Space-time block 779 coded spatial modulation," IEEE Trans. Commun., vol. 59, no. 3, pp. 823- 780 832, Mar. 2011.

[5] M. Di Renzo, H. Haas, and P. M. Grant, "Spatial modulation for multiple- 782 antenna wireless systems: A survey," IEEE Commun. Mag., vol. 49, 783 no. 12, pp. 182-191, Dec. 2011.

[6] M. Di Renzo and H. Haas, "Bit error probability of SM-MIMO over 785 generalized fading channels," IEEE Trans. Veh. Technol., vol. 61, no. 3, 786 pp. 1124-1144, Mar. 2012.

[7] A. Stavridis, S. Sinanovic, M. Di Renzo, and H. Haas, "Energy evaluation 788 of spatial modulation at a multi-antenna base station," in Proc. IEEE Veh. 789 Technol. Conf., Barcelona, Spain, Sep. 2013, pp. 1-5.

[8] N. Serafimovski et al. "Practical implementation of spatial modulation" 791 IEEE Trans. Veh. Technol., vol. 62, no. 9, pp. 511-523, Nov. 2013.

[9] J. Jeganathan, A. Ghrayeb, and L. Szczecinski, "Spatial modulation: 793 Optimal detection and performance analysis," IEEE Commun. Lett., 794 vol. 12 , no. 8, pp. 545-547, Aug. 2008.

[10] S. Sugiura, C. Xu, S. X. Ng, and L. Hanzo, "Reduced complexity coherent 796 versus non-coherent QAM-aided space time shift keying," IEEE Trans. 797 Commun., vol. 59, no. 11, pp. 3090-3101, Nov. 2011.

[11] N. R. Naidoo, H. Xu, and T. Quazi, "Spatial modulation: Optimal detec- 799 tor asymptotic performance and multiple-stage detection," IET Commun., 800 vol. 5, no. 10, pp. 1368-1376, Jul. 2011.

[12] A. Younis, S. Sinanovic, M. Di Renzo, R. Y. Mesleh, and H. Haas, 802 "Generalised sphere decoding for spatial modulation," IEEE Trans. 803 Commun, vol. 61, no. 7, pp. 2805-2815, Jul. 2013.

[13] R. Y. Chang, S.-J. Lin, and W.-H. Chung, "Energy efficient transmis- 805 sion over space shift keying modulated MIMO channels," IEEE Trans. 806 Commun., vol. 60, no. 10, pp. 2950-2959, Oct. 2012.

[14] S. Sanayei and A. Nosratinia, "Antenna selection in MIMO systems," 808 IEEE Commun. Mag., vol. 42, no. 10, pp. 68-73, Oct. 2004.

[15] D. J. Love, R. W. Heath Jr., U. K. N. Lau, D. Gesbert, B. D. Rao, and 810 M. Andrews, "An overview of limited feedback in wireless communica- 811 tion systems," IEEE J. Sel. Areas Commun., vol. 26, no. 8, pp. 1341-1365, 812 Oct. 2008

[16] K. Ntontin, M. Di Renzo, A. Perez-Neira, and C. Verikoukis, "A low- 814 complexity method for antenna selection in spatial modulation systems," 815 IEEE Commun. Lett., vol. 17, no. 12, pp. 2312-2315, Aug. 2013. 
[17] R. Rajashekar, K. V. S. Hari, and L. Hanzo, "Antenna selection in spatia modulation systems," IEEE Commun. Lett., vol. 17, no. 3, pp. 521-524, Mar. 2013.

[18] N. Pillay and H. Xu, "Comments on "Antenna selection in spatial modulation systems'," IEEE Commun. Lett., vol. 17, no. 9, pp. 1681-1683, Sep. 2013.

[19] P. Yang, Y. Xiao, Y. Yi, and S. Li, "Adaptive spatial modulation for wireless MIMO transmission systems," IEEE Commun. Lett., vol. 15, no. 6, pp. 602-604, Jun. 2011.

[20] P. Yang, Y. Xiao, L. Li, Q. Tang, Y. Yu, and S. Li, "Link adaptation for spatial modulation with limited feedback," IEEE Trans. Veh. Technol., vol. 61 , no. 8, pp. 3808-3813, Oct. 2012.

[21] P. Yang, Y. Xiao, Y. Yi, L. Li, Q. Tang, and S. Q. Li, "Simplified adaptive spatial modulation for limited-feedback MIMO," IEEE Trans. Veh. Technol., vol. 62, no. 2, pp. 2656-2666, Jul. 2013.

[22] R. Rajashekar, K. V. S. Hari, and L. Hanzo, "Reduced-complexity ML detection and capacity-optimized training for spatial modulation," IEEE Trans. Commun., vol. 62, no. 1, pp. 112-125, Jan. 2014.

[23] K. Ntontin, M. Di Renzo, A. I. Perez-Neira, and C. Verikoukis, "Adaptive generalized space shift keying," EURASIP J. Wireless Commun. Netw., vol. 43, Feb. 2013.

[24] M. Maleki, H. R. Bahrami, S. Beygi, M. Kafashan, and N. H. Tran, "Space modulation with CSI: Constellation design and performance evaluation," IEEE Trans. Veh. Technol., vol. 64, no. 4, pp. 1623-1634, May 2013.

[25] M. Maleki, H. R. Bahrami, M. Kafashan, and N. H. Tran, "On the performance of spatial modulation: Optimal constellation breakdown," IEEE Trans. Commun., vol. 62, no. 1, pp. 144-157, Jan. 2014.

[26] C. B. Chae, A. Forenza, R. W. Heath Jr., M. R. Mckay, and I. B. Collings, "Adaptive MIMO transmission techniques for broadband wireless communication systems," IEEE Commun. Mag., vol. 48, no. 5, pp. 112-118, May 2010.

[27] L. Collin, O. Berder, P. Rostaing, and G. Burel, "Optimal minimum distance-based precoder for MIMO spatial multiplexing systems," IEEE Trans. Signal Process., vol. 52, no. 5, pp. 617-627, Mar. 2004.

[28] M. Di Renzo and H. Haas, "Improving the performance of space shift keying (SSK) modulation via opportunistic power allocation," IEEE Commun. Lett., vol. 14, no. 6, pp. 500-502, Jun. 2010.

[29] C. Masouros, "Improving the diversity of spatial modulation in MISO channels by phase alignment," IEEE Commun. Lett., vol. 18, no. 5, pp. 729-732, May 2014.

[30] M. Lee, W. Chung, and T. Lee, "Generalized precoder design formulation and iterative algorithm for spatial modulation in MIMO systems with CSIT," IEEE Trans. Wireless Commun., vol. 63, no. 4, pp. 1230-1244, Apr. 2015.

[31] P. Yang, Y. Xiao, B. Zhang, S. Li, M. El-Hajjar, and L. Hanzo, "StarQAM signaling constellations for spatial modulation," IEEE Trans. Veh. Technol., vol. 63, no. 8, pp. 3741-3749, Oct. 2014.

[32] P. Yang, M. Di Renzo, Y. Xiao, S. Li, and L. Hanzo, "Design guidelines for spatial modulation," IEEE Commun. Surveys Tuts., vol. 17, no. 1, pp. 6-26, Mar. 2015

[33] P. Yang, Y. Xiao, S. Li, and L. Hanzo, "A low-complexity power allocation algorithm for multiple-input multiple-output spatial modulation systems," IEEE Trans. Veh. Technol., 2015, to be published, doi: 10.1109/TVT.2015.2410252.

[34] C. Masouros and L. Hanzo, "Constellation-randomization achieves transmit diversity for single-RF spatial modulation," IEEE Trans. Veh. Technol., 2015, to be published.

[35] P. Yang, Y. Xiao, S. Li, and L. Hanzo, "Phase rotation based transmit precoding improves the minimum Euclidean distance of single-RF-chain aided spatial modulation," IET Commun., 2015, to be published.

[36] M. Bazaraa, H. Sherali, and C. Shetty, Nonlinear Programming: Theory and Algorithms. Hoboken, NJ, USA: Wiley, 1993.

[37] S. Chen, N. Ahmad, and L. Hanzo, "Adaptive minimum bit-error rate beamforming," IEEE Trans. Wireless Commun., vol. 4, no. 2, pp. 341348, Mar. 2005.

[38] J. Proakis, Digital Communications. New York, NY, USA: McGraw-Hill, 2001

[39] A. Goldsmith, Wireless Communications. Cambridge, U.K.: Cambridge Univ. Press, 2005.

[40] D. Yang, C. Xu, L.-L. Yang, and L. Hanzo, "Transmit diversity-assisted space-shift keying for collocated and distributed/cooperative MIMO elements," IEEE Trans. Veh. Technol., vol. 60, no. 6, pp. 2864-2869, Jul. 2011.

[41] T. Handte, A. Muller, and J. Speidel, "BER analysis and optimization of generalized spatial modulation in correlated fading channels," in Proc. IEEE Veh. Technol. Conf., Anchorage, AK, USA, Sep. 2009, pp. 1-5.

[42] E. Başar, Ü. Aygölü, E. Panayırcı, and H. V. Poor, "Performance of spatial 894 modulation in the presence of channel estimation errors," IEEE Commun. 895 Lett., vol. 16, no. 2, pp. 176-179, Feb. 2012.

[43] S. S. Ikki and R. Mesleh, "A general framework for performance analy- 897 sis of space shift keying (SSK) modulation in the presence of Gaussian 898 imperfect estimations," IEEE Commun. Lett., vol. 16, no. 2, pp. 228-230, 899 Feb. 2012

[44] N. Wang, W. Liu, H. Men, M. Jin, and H. Xu. "Further complexity reduc- 901 tion using rotational symmetry for EDAS in spatial modulation," IEEE 902 Commun. Lett., vol. 18, no. 10, pp. 1835-1838, Oct. 2014.

[45] J. Zheng and J. Chen, "Further complexity reduction for antenna selec- 904 tion in spatial modulation systems," IEEE Commun. Lett., vol. 19, no. 6, 905 pp. 937-940, Jun. 2015, doi: 10.1109/LCOMM.2015.2417884.

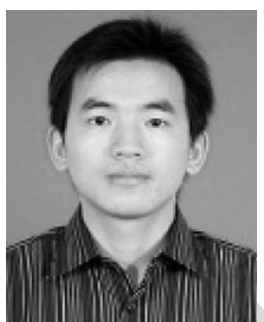

Ping Yang received the B.E. and M.E. degrees 907 from the University of Electronic Science and 908 Technology of China (UESTC), Chengdu, China, in 909 2006 and 2009, respectively. From September 2012910 to September 2013, he was a Visiting Student at 911 the School of Electronics and Computer Science, 912 University of Southampton, Southampton, U.K. 913 From May 2014, he is a Research Fellow with 914 EEE, NTU, Singapore. Also, he is an Assistant 915 Professor with UESTC. His research interests include 916 MIMO/OFDM, machine learning, life science, and 917 communication signal processing.

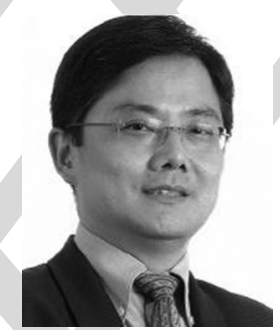

storage systems.

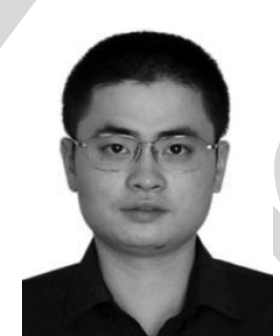

Yong Liang Guan (S'94-AM'97-M'99) received 919 the Ph.D. degree from the Imperial College of 920 Science, Technology and Medicine, University of 921 London, London, U.K., and the B.Eng. degree (with 922 first class Hons.) from the National University of 923 Singapore, Singapore, in 1997 and 1991, respectively. 924 $\mathrm{He}$ is now an Associate Professor with the School 925 of Electrical and Electronic Engineering, Nanyang 926 Technological University, Singapore. His research 927 interests include modulation, coding and signal pro- 928 cessing for communication, information security, and 929

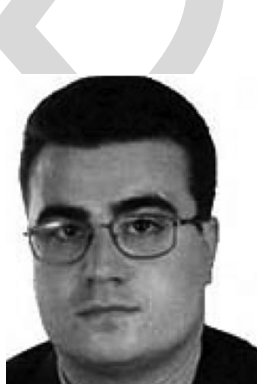

Yue Xiao received the Ph.D. degree in communi- 931 cation and information systems from the University 932 of Electronic Science and Technology of China, 933 Chengdu, China, in 2007. He is now a Full Professor 934 with the University of Electronic Science and 935 Technology of China. He has authored more than 30936 international journals and been involved in several 937 projects in the Chinese Beyond 3G Communication 938 R\&D Program. His research interests include wire- 939 less and mobile communications.

Marco Di Renzo (S'05-AM'07-M'09-SM'14) was 941 born in L'Aquila, Italy, in 1978. He received the 942 Laurea (cum laude) and Ph.D. degrees in electri- 943 cal and information engineering from the University 944 of L'Aquila, L'Aquila, Italy, in April 2003 and 945 January 2007, respectively. Since January 2010, he 946 has been a Tenured Academic Researcher ("Chargé 947 de Recherche Titulaire") with the French National 948 Center for Scientific Research (CNRS), as well as 949 a Faculty Member of the Laboratory of Signals and 950 Systems (L2S), a joint Research Laboratory of the 951 CNRS, the École Supérieure d'Électricité (SUPÉLEC) and the University of 952 Paris-Sud XI, Paris, France. His research interests include wireless communi- 953 cations theory. Currently, he serves an Editor of the IEEE COMMUNICATIONS 954 LETTERS and the IEEE TRANSACTIONS ON COMMUNICATIONS. He is the 955 recipient of a special mention for the 2012 IEEE CAMAD Best Paper Award; 956 the 2013 Network of Excellence NEWCOM\# Best Paper Award; the 2013957 IEEE-COMSOC Best Young Researcher Award for Europe, Middle East and 958 Africa (EMEA Region); and the 2014 IEEE ICNC Single Best Paper Award 959 Nomination (Wireless Communications Symposium).

\section{(1) ,

. ,

,
, 
961

962

963

964

965

966

967

968

969

970

971

972

973

974

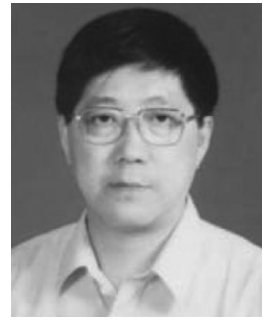

Shaoqian Li (AM'04-SM'12) received the B.S.E. degree in communication technology from Northwest Institute of Telecommunication, Xidian University, Xidian, China, and the M.S.E. degree in communication system from the University of Electronic Science and Technology of China (UESTC), Chengdu, China, in 1982 and 1984, respectively. He is a Professor, Ph.D Supervisor, Director of the National Key Laboratory of Communication, UESTC, and Member of the National High Technology R\&D Program (863 Program) Communications Group. His research interests include wireless communication theory, antiinterference technology for wireless communications, spread-spectrum and frequency-hopping technology, mobile, and personal communications.

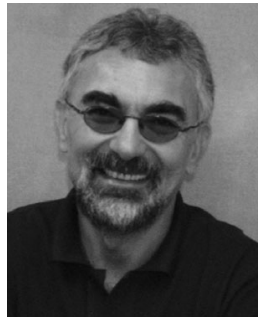

Lajos Hanzo (M'91-SM'92-F'04) received the 975 D.Sc. degree in electronics and the doctorate degree, 976 in 1976 and 1983, respectively. During his 37-year 977 career in telecommunications, he has held various 978 research and academic posts in Hungary, Germany, 979 and the U.K. Since 1986, he has been with the School 980 of Electronics and Computer Science, University of 981 Southampton, Southampton, U.K, where he holds 982 the Chair in Telecommunications. He has success- 983 fully supervised 80+ Ph.D. students, coauthored 20984 Wiley/IEEE Press books on mobile radio communi- 985 cations totaling in excess of 10000 pages, authored 1400+ research entries at 986 IEEE Xplore, acted both as TPC and General Chair of the IEEE conferences, 987 presented keynote lectures and has been awarded a number of distinctions. 988 Currently, he is directing a 100-strong academic research team, working on 989 a range of research projects in the field of wireless multimedia communica- 990 tions sponsored by industry, the Engineering and Physical Sciences Research 991 Council (EPSRC) U.K., the European Research Council's Advanced Fellow 992 Grant and the Royal Society's Wolfson Research Merit Award. He is also a 993 Governor of the IEEE VTS. From 2008 to 2012, he was the Editor-in-Chief of 994 the IEEE Press and a Chaired Professor also at Tsinghua University, Beijing. 995 His research is funded by the European Research Council's Senior Research 996 Fellow Grant. He served as FREng, FIEEE, FIET, a Fellow of EURASIP. In 997 2009, he was the recipient of the honorary doctorate "Doctor Honoris Causa" 998 by the Technical University of Budapest, Budapest, Hungary. 


\section{QUERIES}

Q1: Please be advised that per instructions from the Communications Society this proof was formatted in Times Roman font and therefore some of the fonts will appear different from the fonts in your originally submitted manuscript. For instance, the math calligraphy font may appear different due to usage of the usepackage[mathcal]euscript. We are no longer permitted to use Computer Modern fonts.

Q2: Please provide page range for Ref. [23].

Q3: Please provide volume number, issue number, and page range for Refs. [33], [34], and [35]. 


\title{
Transmit Precoded Spatial Modulation: Maximizing the Minimum Euclidean Distance Versus Minimizing the Bit Error Ratio
}

\author{
Ping Yang, Yong Liang Guan, Member, IEEE, Yue Xiao, Marco Di Renzo, Senior Member, IEEE, \\ Shaoqian Li, Senior Member, IEEE, and Lajos Hanzo, Fellow, IEEE
}

Abstract-In this paper, we investigate a pair of transmit precoding (TPC) algorithms conceived for spatial modulation (SM) systems communicating over flat-fading multiple-input multiple-output (MIMO) channels. In order to retain all the benefits of conventional SM, we design the TPC matrix to be diagonal and introduce two design criteria for optimizing the elements of the TPC matrix. Specifically, we first investigate a TPC design based on maximizing the minimum Euclidean distance $d_{\min }\left(\max -d_{\min }\right)$ between the SM signal points at the receiver side. A closed-form solution of the optimal max- $d_{\text {min }}$-based TPC matrix is derived. Then, another TPC design algorithm is proposed for directly minimizing the bit error ratio (BER) upper bound of SM, which is capable of jointly optimizing the overall Euclidean distance between all received signal points. In the minimum BER (min-BER)-based TPC algorithm, the theoretical gradient of the BER with respect to the diagonal TPC matrix is derived and a simplified iterative conjugate gradient (SCG) algorithm is invoked for TPC optimization. Our simulation results demonstrate that the proposed max- $d_{\min }$-based TPC algorithm is optimal in terms of the minimum distance. However, increasing $d_{\min }$ does not achieve a further BER improvement. We also confirm that the min-BERbased TPC outperforms the max- $d_{\text {min }}$-based TPC schemes in terms of the achievable BER performance.

Manuscript received March 23, 2015; revised November 2, 2015; accepted November 2, 2015. This work was supported in part by the National Science Foundation of China under Grant 61471090, in part by the National Basic Research Program of China under Grant 2013CB329001, in part by the National High-Tech R\&D Program of China ("863" Project under Grant 2014AA01A707), in part by the Foundation Project of National Key Laboratory of Science and Technology on Communications under Grant 9140C020108140C02005, and in part by the European Research Council's Advanced Fellow Grant are gratefully acknowledged. The work of M. D. Renzo was supported by the European Commission under the auspices of the FP7-PEOPLE MITN-CROSSFIRE project (grant 317126). The associate editor coordinating the review of this paper and approving it for publication was Z. Wang.

P. Yang is with the National Key Laboratory of Science and Technology on Communications, University of Electronic Science and Technology of China, Sichuan 611731, China, and also with the School of Electrical and Electronic Engineering, Nanyang Technological University, Singapore (e-mail: yang.ping@uestc.edu.cn).

Y. L. Guan is with the School of Electrical and Electronic Engineering, Nanyang Technological University, Singapore (e-mail: eylguan@ntu.edu.sg).

Y. Xiao and $\mathrm{S}$. Li are with the National Key Laboratory of Science and Technology on Communications, University of Electronic Science and Technology of China, Sichuan 611731, China (e-mail: xiaoyue@uestc.edu.cn; lsq@yeste.edu.cn).

M. D. Renzo is with the Laboratory of Signals and Systems (L2S), University of Paris-Sud XI, Orsay, France (e-mail: marco.direnzo@ 1ss.supelec.fr).

L. Hanzo is with the School of Electronics and Computer Science, University of Southampton, Southampton SO17 1BJ, U.K. (e-mail: 1h@ecs.soton.ac.uk).

Color versions of one or more of the figures in this paper are available online at http://ieeexplore.ieee.org.

Digital Object Identifier 10.1109/TWC.2015.2497692
Index Terms-Minimum Euclidean distance, minimum BER, multiple-input multiple-output, pre-coding technique, spatial modulation.

\section{INTRODUCTION}

$\mathbf{R}$ ECENTLY, spatial modulation (SM) has been proposed as a new class of low-complexity energy-efficient multiple-input multiple-output (MIMO) approach, whilst relying on a single-radio frequency (RF) chain [1]-[3]. SM scheme relies on the unique encoding philosophy of activating one out of $N_{t}$ transmit antennas (TAs) during each transmission slot [4]. The activated TA then transmits classic complex-valued symbols of amplitude and phase modulation (APM). The potential benefits of SM over the conventional MIMO techniques have been validated not only via numerical simulations [5], [6] but also by laboratory experiments [7], [8].

Early work has been focused on low-complexity receiver designs conceived for minimizing the bit error ratio (BER) of SM [9]-[12]. It was shown in [9] that a low-complexity single-stream maximum likelihood (ML) detector or an even lower-complexity near-ML detector [10]-[12] is capable of striking a beneficial trade-off amongst the potentially conflicting factors of energy-efficiency, multiplexing gain and diversity gain compared to other MIMO transmission techniques [13]. In addition to a plethora of receivers, preprocessing at the transmitter has also been conceived for achieving a further performance improvement. Specifically, several antenna selection (AS) methods [14], [15] originally designed for conventional MIMO systems have also been generalized for employment in SM systems with the goal of enhancing its capacity or BER performance [16]-[18]. In [19]-[21], an adaptive SM (ASM) scheme was proposed for improving the achievable BER, while maintaining the target throughput with the aid of adaptive modulation (AM) techniques. In [22], the power allocation between the pilot and data was optimized for maximizing the capacity of SM transmission. In [23] and [24], a specific constellation design was proposed for space shift keying (SSK) systems in order to improve their BER. The constellation design was further developed in [25] for SM by finding the optimal combination of the number of TAs and the APM size that minimizes the BER.

Among the promising design alternatives, linear transmit pre-coding (TPC) techniques constitute an attractive transmit preprocessing regime, since they use a simple matrix $\mathbf{U}$ for 


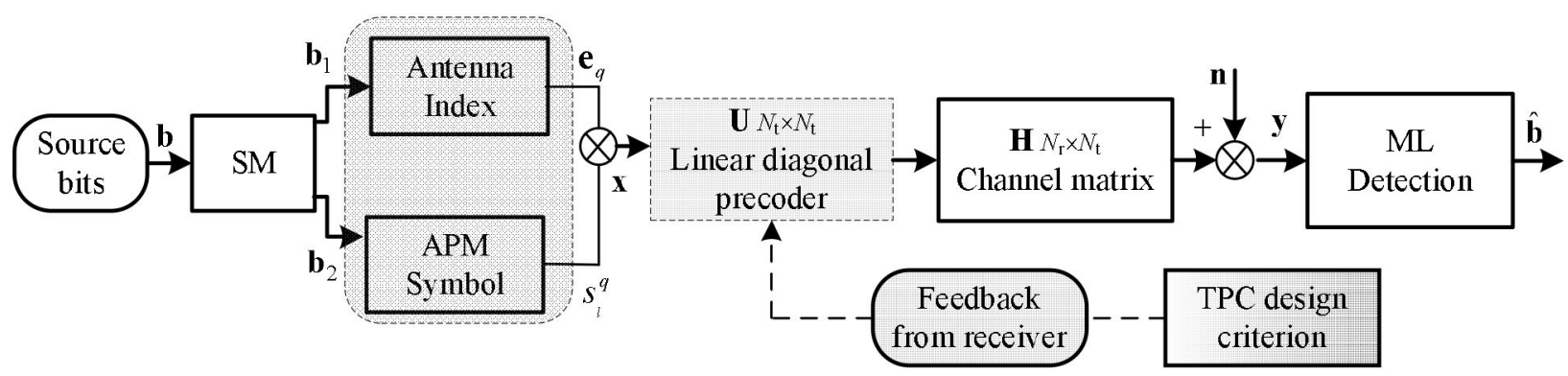

Fig. 1. The diagonal TPC based SM transmission system.

weighting the channel matrix in order to enhance the attainable performance [26]. Indeed, TPC has been widely researched in the context of classic spatial multiplexing systems [27]. However, since only a single TA is activated in each time slot in SM, these TPC approaches are not directly suitable for SM systems.

In [28] the effect of power imbalance has been researched in the context of SSK associated with TPC algorithms. More recently, the research efforts have been focused on the TPC design of SM based on maximizing the minimum Euclidean distance $d_{\min }\left(\max -d_{\min }\right)$ in the received SM constellation. In [29], the phase alignment technique has also been extended to SM systems for constellation shaping in order to provide BER benefit in multiple-input single-output (MISO) channels. In [30], the max- $d_{\min }$ based TPC is designed by using an iterative concave-convex process, where the TPC parameters are calculated for each transmit constellation points. In [31], [32], a diagonal TPC was proposed for maximizing $d_{\min }$ in SM systems. However, an exhaustive numerical search method was used for identifying the specific TPC parameters. On the other hand, in [33], a special case of the diagonal TPC, namely an adaptive power allocation (PA) method, was investigated, where a simple real-valued diagonal TPC matrix was considered. As shown in [33], closed-form solutions of the optimal max $-d_{\min }$ aided PA were derived in the case of two TAs. In [34], another diagonal TPC method, namely phase rotation precoding (PRP), was proposed for energy-efficient transmission, where only the phases of the SM symbols were optimized based on the $\max -d_{\min }$ criterion. The corresponding closed-form solutions were derived for two TAs and for PSK-modulated SM schemes in [35].

Against this background, the novel contributions of this paper are as follows.

1) We first investigate a general TPC matrix design algorithm based on the max- $d_{\min }$ criterion, where a complexvalued TPC matrix is considered instead of the realvalued PA matrix of [33] and the constant-modulus PRP matrix of [34], [35]. Compared to the heuristic method of computing the TPC matrix of [31], in this paper we derive closed-form solutions of the $\max -d_{\min }$ TPC for a $\left(2 \times N_{r}\right)$-element BPSK-modulated SM scheme as well as for the more general cases of $M$-PSK modulated $(2 \times$ $N_{r}$ )-element SM. Moreover, we extend this method to the case of $N_{t}>2$.

2) It is shown that the max- $d_{\text {min }}$ based TPC-aided SM scheme is capable of achieving a larger $d_{\min }$ than other max $-d_{\text {min }}$ aided adaptive SM schemes, such as the PA 118 based TPC-aided SM scheme of [33] and the ASM 119 scheme of [21]. However an increase of $d_{\min }$ does not 120 achieve a further BER improvement. We find the reason 121 that the $\max -d_{\min }$ TPC only has a higher the mini- 122 mum received distance $d_{\text {min }}$ and may result in reduced 123 Euclidean distances between the non-adjacent received 124 constellation points.

3) To alleviate this shortcoming, we propose a new 126 minimum-BER (min-BER) based TPC method, which is 127 capable of jointly optimizing the overall Euclidean dis- 128 tance between the received signal points. Specifically, the 129 theoretical gradient of the BER upper bound of SM with 130 respect to the diagonal TPC matrix $\mathbf{U}$ is derived, and the 131 simplified conjugate gradient (SCG) algorithm [36], [37] 132 is invoked for efficient TPC optimization. We demon- 133 strate that the overall BER gain of the proposed method 134 is significantly improved compared to both that of con- 135 ventional SM and to the other existing TPC schemes of 136 [29]-[35]. We also extend the proposed algorithm to cope 137 with channel state information (CSI) inaccuracies. 138

The organization of the paper is as follows. Section II intro- 139 duces the concept as well as the system model of the TPC-based 140 SM. In Section III and Section IV, we present a pair of TPC 141 designs conceived for enhancing the BER performance of SM. 142 The complexity analysis results are provided in Section V. Our 143 Simulation results and performance comparisons are presented 144 in Section VI. Finally, Section VII concludes the paper. 145

Notation: $(\cdot)^{*},(\cdot)^{T}$ and $(\cdot)^{H}$ denote conjugate, transpose, and 146 Hermitian transpose, respectively. The probability of an event is 147 represented by $P(\cdot)$. Furthermore, $\|\cdot\|$ stands for the Frobenius 148 norm and all logarithms are base of $2 . \operatorname{Tr}(\cdot)$ denotes the trace of 149 a square matrix, $E(\cdot)$ represents expectation, while $\operatorname{Re}\{\mathbf{x}\}$ and 150 $\operatorname{Im}\{\mathbf{x}\}$ represent the real and imaginary parts of $\mathbf{x}$, respectively. 151 $\mathbf{I}_{b}$ denotes a $(b \times b)$-element identity matrix and the operator 152 $\operatorname{diag}\{\cdot\}$ to be applied to a length $i$ vector returns an $i \times i$ square 153 matrix with the vector elements along the diagonal.

\section{SySTEM MODEL}

\section{A. Signal Model of the Diagonal TPC Aided SM-MIMO}

Consider a MIMO system having $N_{t}$ transmit and $N_{r}$ receive 157 antennas. In this paper, $N_{t}$ is assumed to be a power of two. 158 Let $\mathbf{b}=\left[b_{1}, \ldots, b_{L}\right]$ be the transmit bit vector of each time 159 slot, which contains $L=\log _{2}\left(N_{t} M\right)$ bits. As shown in Fig. 1, 160 the input vector $\mathbf{b}$ is divided into two sub-vectors of $\log _{2}\left(N_{t}\right) 161$ 
and $\log _{2}(M)$ bits, denoted as $\mathbf{b}_{1}$ and $\mathbf{b}_{2}$, respectively. The bits in the sub-vector $\mathbf{b}_{1}$ are used for selecting a unique TA index $q$ for activation, which is mapped to a $N_{t}$-dimensional standard basis vector $\mathbf{e}_{q}\left(1 \leq q \leq N_{t}\right)$. The bits in the sub-vector $\mathbf{b}_{2}$ are mapped to a Gray-coded APM symbol $s_{m}^{q} \in \mathfrak{I}(m \in$ $\{1, \ldots, M\})$ [38]. Then, the resultant SM symbol $\mathbf{x} \in \mathbb{C}^{N_{t} \times 1}$ can be formulated as [1]

$$
\mathbf{x}=s_{m}^{q} \mathbf{e}_{q}=\left[0, \ldots, s_{m}^{q}, \ldots, 0\right] .
$$

As shown in Fig. 1, after TPC relying on the linear diagonal matrix $\mathbf{U}$, the signal observed at the $N_{r}$ receive antennas is given by

$$
\mathbf{y}=\mathbf{H U x}+\mathbf{n},
$$

where $\mathbf{H}$ is the $\left(N_{r} \times N_{t}\right)$-element channel matrix, $\mathbf{U}$ is the $\left(N_{t} \times N_{t}\right)$-element TPC matrix, and $\mathbf{n}$ is the $\left(N_{r} \times 1\right)$-element noise vector. We assume $E\left[\mathbf{n n}^{H}\right]=N_{0} \mathbf{I}_{N_{r}}$ and $E\left[\mathbf{x n}^{H}\right]=$ $\mathbf{0}_{N_{t} \times N_{t}}$. The elements of the noise vector $\mathbf{n}$ are complex Gaussian random variables obeying $\mathcal{C N}\left(0, N_{0}\right)$. Furthermore, the diagonal TPC matrix $\mathbf{U}$ is given by

$$
\mathbf{U}=\operatorname{diag}\left\{u_{1}, \ldots, u_{q}, \ldots, u_{N_{t}}\right\},
$$

where $u_{q}$ is a complex-valued TPC parameter, which controls the channel gain associated with $x_{q}$. We enforce the constraint $\sum_{q=1}^{N_{t}}\left|u_{q}\right|^{2}=P_{\mathrm{T}}$ for the sake of normalizing the transmit power. The diagonal structure of $\mathbf{U}$ guarantees that the transmit vector $\mathbf{U x}$ has a single non-zero component, hence the single-RF-chain benefits (such as the avoidance of both the inter antenna interference (IAI) and of the multiple RFs) of SM are preserved. The matrix $\mathbf{U}$ can be decomposed as follows [31]

$$
\mathbf{U}=\mathbf{P} \Theta=\operatorname{diag}\left\{p_{1}, p_{2} e^{j \theta_{1}}, \ldots, p_{q} e^{j \theta_{q-1}}, \ldots, p_{N_{t}} e^{j \theta_{N_{t}-1}}\right\},
$$

where $u_{q}=p_{q} e^{j \theta_{q-1}}$ and $p_{q}$ represents the complex modulus of $u_{q}$, while $\theta_{q-1}$ represents the phase angle of $u_{q}$. In (4), the TPC matrix $\mathbf{U}$ is decomposed into two matrices related to the modulus and phase, which correspond to the real-valued PA matrix $\mathbf{P}=\operatorname{diag}\left\{p_{1}, p_{2}, \ldots, p_{q}, \ldots, p_{N_{t}}\right\}$ and to the complexvalued PRP matrix $\boldsymbol{\Theta}=\operatorname{diag}\left\{1, e^{j \theta_{1}}, \ldots, e^{j \theta_{q-1}}, \ldots, e^{j \theta_{N_{t}-1}}\right\}$, respectively.

Remark: The max- $d_{\min }$ based PA-aided SM schemes of [33] and the PRP-aided SM schemes of [34], [35] constitute special cases of the proposed TPC schemes, which can be obtained by setting $\boldsymbol{\Theta}=\mathbf{I}_{N_{t}}$ and $\mathbf{P}=\mathbf{I}_{N_{t}}$, respectively.

\section{B. Maximum Likelihood Receiver}

The receiver performs ML detection over all possible SM symbols $\mathbf{x} \in \mathbb{C}^{N_{t} \times 1}$ for retrieving the transmit symbols, which can be formulated as [9], [10]:

$$
\begin{aligned}
\hat{\mathbf{x}} & =\arg \min _{\mathbf{x} \in \mathbb{X}}\|\mathbf{y}-\mathbf{H U} \mathbf{x}\|^{2} \\
& =\arg \min _{\mathbf{x} \in \mathbb{X}}\|\mathbf{y}-\tilde{\mathbf{H}} \mathbf{x}\|^{2}=\arg \min _{q \in\left\{1, \ldots, N_{t}\right\}}\left\|\mathbf{y}-\tilde{\mathbf{h}}_{q} s_{m}^{q}\right\|^{2} \\
& \Leftrightarrow \arg \min _{q \in\left\{1, \ldots, N_{t}\right\}}\left\|\tilde{\mathbf{h}}_{q}^{H} \mathbf{y} /\right\| \tilde{\mathbf{h}}_{q}\left\|^{2}-s_{m}^{q}\right\|^{2},
\end{aligned}
$$

where $\mathbb{X}$ is the set of all legitimate transmit symbols and $\tilde{\mathbf{h}}_{q}$ is 201 the $q-t h$ column of the equivalent channel matrix $\tilde{\mathbf{H}}=\mathbf{H U} . \quad 202$ As shown in Eq. (5), a low-complexity single-stream ML detec- 203 tor is obtained [10], [22]. Moreover, it is shown in Proposition 204 1 of [22] that for a square- or for a rectangular-QAM constella- 205 tion, the complexity imposed is independent of the constellation 206 size, and that it increases only with $N_{t}$. 207

The conditional error performance of a ML receiver for 208 a given channel $\mathbf{H}$ can be approximated by the sum of the 209 pairwise error probability (PEP) [39], which is given by 210

$$
P(\mathbf{H}) \leq \frac{1}{L} \sum_{i=1}^{L} \sum_{\substack{j=1, i \neq j}}^{L} Q\left(\sqrt{\frac{1}{2 N_{0}} d_{i j}(\mathbf{H})}\right),
$$

where $Q(x)=(1 / \sqrt{2 \pi}) \int_{x}^{\infty} e^{-y^{2} / 2} d y$ denotes the Gaussian 211 tail probability, while the distance $d_{i j}(\mathbf{H})$ at the receiver is 212 defined as

$$
\begin{aligned}
d_{i j}(\mathbf{H}) & =\left\|\mathbf{H U}\left(\mathbf{x}_{i}-\mathbf{x}_{j}\right)\right\|^{2} \\
& =\left\|\mathbf{H} \mathbf{U} \mathbf{e}_{i j}\right\|^{2},
\end{aligned}
$$

where $\mathbf{e}_{i j}=\mathbf{x}_{i}-\mathbf{x}_{j}, i \neq j$ denotes the error vector. The PEP 214 depends on the specific SM symbol pair $\left(\mathbf{x}_{i}, \mathbf{x}_{j}\right)$, on the instan- 215 taneous channel realization $\mathbf{H}$ and the TPC matrix $\mathbf{U}$.

\section{Minimum Euclidean Distance Based TPC}

It follows by direct inspection of the PEP expression of 218 Eq. (6) that the performance of the ML receiver is pre- 219 dominantly affected by the distances $d_{i j}(\mathbf{H})$. Motivated by 220 this observation, TPC design methods based on maximiz- 221 ing the minimum value of $d_{i j}(\mathbf{H})$ (the distance $d_{\min }$ ) have 222 been introduced in [31] and [32]. However, only a high- 223 complexity numerical approach was proposed for optimizing 224 the TPC matrix. In this section, we first briefly introduce the 225 max $-d_{\min }$ based TPC method. Then, we derive the related 226 solutions.

\section{A. Design Criterion}

At high SNR, Eq. (6) can be further simplified as follows [39]

229

$$
P(\mathbf{H}) \leq \lambda \cdot Q\left(\sqrt{\frac{1}{2 N_{0}} d_{\min }}\right),
$$

where $\lambda$ is the number of neighbor points [39] and $d_{\min }$ is 230 defined as

$$
d_{\min }=\min _{\substack{i, j \\ i \neq j}} d_{i j}(\mathbf{H})=\min _{\mathbf{e}_{i j} \in \mathbb{E}}\left\|\mathbf{H} \mathbf{U} \mathbf{e}_{i j}\right\|^{2} .
$$

In Eq. (8), $P(\mathbf{H})$ is a monotonically decreasing function of 232 $d_{\min }$. Hence, the system's BER performance may be improved 233 by maximizing the distance $d_{\min }$ of the received constellation 234 upon carefully adapting the TPC matrix $\mathbf{U}$ under the power 235 
constraint $P_{\mathrm{T}} .{ }^{1}$ Based on this principle, the max $-d_{\min }$ based TPC matrix $\mathbf{U}$ design rule can be formulated as follows

$$
\begin{aligned}
& \mathbf{U}_{\text {opt }}=\underset{\mathbf{U}}{\arg \max } d_{\min } \\
& \text { s.t. } \quad \operatorname{tr}\left(\mathbf{U U}^{T}\right) \leq P_{\mathrm{T}} .
\end{aligned}
$$

Note that in [33] and [35] the closed-form solutions for two special cases of Eq. (10), namely for the $\max -d_{\min }$ based PA matrix and for the max $-d_{\min }$ based PRP matrix, have been derived. However, to the best of our knowledge, the closedform solution for the joint design of the PA and PRP of Eq. (10) has not been reported in the existing literature. In the following subsections, we derive a closed-form solution for the TPC matrix of the BPSK-modulated $\left(2 \times N_{r}\right)$-element SM and extend the method to the more general $M$-PSK modulated $\left(2 \times N_{r}\right)$-element SM arrangements. Additionally, as shown in [2] and [6], PSK schemes are preferred over QAM schemes in SM. Hence, PSK is adopted in this paper.

\section{B. Optimal TPC Matrix for BPSK-modulated $2 \times N_{r} S M$}

Let us consider a BPSK-modulated SM systems associated with $N_{t}=2$, where the BPSK symbols belong to the set $\mathfrak{s}=\{1,-1\}$, and all possible error vectors $\mathbf{e}_{i j}=\mathbf{x}_{i}-\mathbf{x}_{j}, i \neq$ $j$ are listed as follows: $\left\{[-2,0]^{T},[2,0]^{T},[0,-2]^{T},[0,2]^{T}\right.$, $\left.[-1,1]^{T},[-1,-1]^{T},[1,-1]^{T},[1,1]^{T}\right\}$. Similar to the method of [33], since some vectors are collinear, the set to be studied is reduced to $\tilde{\mathbb{E}}_{B P S K}=\left\{\mathbf{e}_{1}, \mathbf{e}_{2}, \mathbf{e}_{3}, \mathbf{e}_{4}\right\}=\left\{[2,0]^{T},[0,2]^{T}\right.$, $\left.[1,-1]^{T},[1,1]^{T}\right\}$. Given the channel matrix $\mathbf{H}=\left[\mathbf{h}_{1}, \mathbf{h}_{2}\right]$ and the corresponding TPC matrix $\mathbf{U}=\operatorname{diag}\left\{p_{1}, p_{2} e^{j \theta_{1}}\right\}$, the distances at the receiver based on Eq. (7) are given by

$$
\left\{\begin{aligned}
d_{1} & =\left\|\mathbf{H U} \mathbf{e}_{1}\right\|^{2}=4\left\|p_{1} \mathbf{h}_{1}\right\|^{2} \\
d_{2} & =\left\|\mathbf{H U} \mathbf{e}_{2}\right\|^{2}=4\left\|p_{2} \mathbf{h}_{2}\right\|^{2} \\
d_{3} & =\left\|\mathbf{H U} \mathbf{e}_{3}\right\|^{2}=\left\|p_{1} \mathbf{h}_{1}-p_{2} e^{j \theta_{1}} \mathbf{h}_{2}\right\|^{2} \\
d_{4} & =\left\|\mathbf{H U} \mathbf{e}_{4}\right\|^{2}=\left\|p_{1} \mathbf{h}_{1}+p_{2} e^{j \theta_{1}} \mathbf{h}_{2}\right\|^{2}
\end{aligned}\right.
$$

Based on the distances in Eq. (11), the optimization problem of Eq. (10) can be modified as follows

$$
\begin{aligned}
& \mathbf{U}_{\text {opt }}=\underset{\mathbf{U}}{\arg \max }\left\{\min \left\{d_{1}, d_{2}, d_{3}, d_{4}\right\}\right\} \\
& \text { s.t. } \quad \operatorname{tr}\left(\mathbf{U} \mathbf{U}^{T}\right) \leq P_{\mathrm{T}}
\end{aligned}
$$

To obtain the specific TPC matrix $\mathbf{U}_{\text {opt }}$, which maximizes the distance $d_{\min }$, the parameters $p_{1}, p_{2}$ and $\theta_{1}$ in Eq. (12) have to be computed. As indicated in Eq. (11) and shown in Fig. 2, for a fixed PA matrix $\mathbf{P}=\operatorname{diag}\left\{p_{1}, p_{2}\right\}, d_{1}$ and $d_{2}$ are independent of the phase $\theta_{1}$, while $d_{3}$ and $d_{4}$ are given by sinusoidal functions of the phase $\theta_{1}$. In order to find the optimal phase solution $\theta_{1}^{o p t}$, we can first obtain the phases assigned to

\footnotetext{
${ }^{1}$ Compared to the PRP method of [34], [35], the power of the SM symbols may indeed fluctuate due to the TPC algorithm. However, during the time when the channel envelope remains constant within its coherence-interval, the power values of the transmit symbols are selected from a finite discrete set. In practice, the constraint $P_{\mathrm{T}}$ should be carefully selected according to the system requirements, such as the peak to average power ratio (PAPR) and the BER metrics.
}

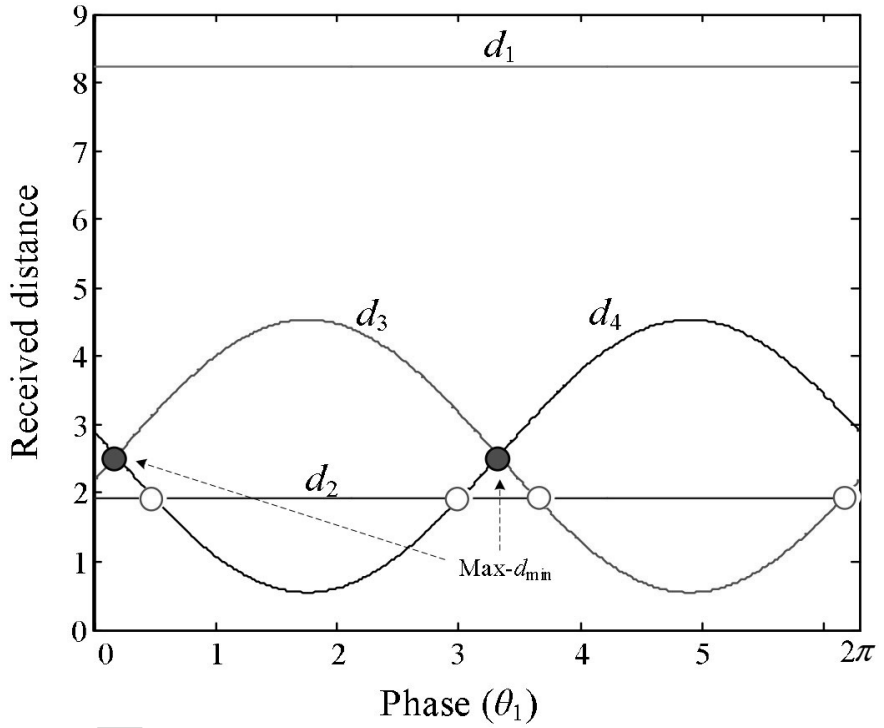

Fig. 2. The phase solutions of the BPSK-modulated $\left(2 \times N_{r}\right)$-element TPC based SM for a fixed PA.

the TAs by finding the intersections of the sinusoidal curves in 270 Fig. 2, and then continue by computing the optimal PA matrix 271 as $\mathbf{P}=\operatorname{diag}\left\{p_{1}^{o p t}, p_{2}^{o p t}\right\}$.

To be specific, as shown in Fig. 2, regardless of the specific 273 PA matrix $\mathbf{P}$, the optimal phase $\theta_{1}^{\text {opt }}$ that maximizes $d_{\min }$ should 274 satisfy the constraint of $d_{3}=d_{4}$ which from (11):

$$
\left\|p_{1} \mathbf{h}_{1}-p_{2} e^{j \theta_{1}^{o p t}} \mathbf{h}_{2}\right\|^{2}=\left\|p_{1} \mathbf{h}_{1}+p_{2} e^{j \theta_{1}^{o p t}} \mathbf{h}_{2}\right\|^{2}
$$

Eq. (13) can be further simplified to $\operatorname{Re}\left\{\mathbf{h}_{1}^{H} \mathbf{h}_{2} e^{j \theta_{1}^{o p t}}\right\}=0.276$ Upon introducing the shorthands $a=\left\|\mathbf{h}_{1}\right\|^{2}, b=\left\|\mathbf{h}_{2}\right\|^{2}, c_{1}=277$ $2 \cdot \operatorname{Re}\left\{\mathbf{h}_{1}^{H} \mathbf{h}_{2}\right\}$ and $c_{2}=2 \cdot \operatorname{Im}\left\{\mathbf{h}_{1}^{H} \mathbf{h}_{2}\right\}$ for a given channel 278 matrix H, Eq. (13) can be solved as

$$
\begin{aligned}
& c_{1} \cos \theta_{1}^{o p t}-c_{2} \sin \theta_{1}^{o p t}=0 \\
& \Leftrightarrow \theta_{1}^{o p t}=k \pi+\tan ^{-1}\left(\frac{c_{1}}{c_{2}}\right), k \in \mathbb{Z}, 0 \leq \theta_{1}^{o p t} \leq 2 \pi .
\end{aligned}
$$

Given the phase solution of Eq. (14), the distances $d_{3}$ and $d_{4} 280$ of Eq. (11) are simplified as follows

$$
d_{3}=d_{4}=\left\|p_{1} \mathbf{h}_{1}\right\|^{2}+\left\|p_{2} \mathbf{h}_{2}\right\|^{2}=a p_{1}^{2}+b p_{2}^{2} .
$$

To compute the optimal PA parameters as $p_{1}^{o p t}$ and $p_{2}^{o p t}, 282$ since we have $d_{3}=d_{4}$ in Eq. (15), the optimization problem 283 of Eq. (12) can be further simplified to:

$$
\begin{aligned}
\mathbf{U}_{\mathrm{opt}}=\underset{\mathbf{U}}{\arg \max }\left\{\min \left\{d_{1}, d_{2}, d_{3}\right\}\right\} \\
=\underset{\mathbf{U}}{\arg \max }\left\{\operatorname { m i n } \left\{4 a p_{1}^{2}, 4 b\left(P_{\mathrm{T}}-p_{1}^{2}\right),\right.\right. \\
\left.\left.\quad a p_{1}^{2}+b\left(P_{\mathrm{T}}-p_{1}^{2}\right)\right\}\right\} .
\end{aligned}
$$

As indicated in Eq. (16), $d_{1}, d_{2}$ and $d_{3}$ are linear functions 285 of the parameter $\mu_{1}=p_{1}^{2}$. Hence, the max- $d_{\min }$ solution given 286 
$\mu_{1}$ is one of the intersections between these distances $d_{i}(i=$ $1,2,3)$, which are given by

$$
\left\{\begin{array}{l}
p_{1}^{(1)}=\sqrt{b /(a+b) P_{\mathrm{T}}} \\
p_{1}^{(2)}=\sqrt{b /(3 a+b) P_{\mathrm{T}}} \\
p_{1}^{(3)}=\sqrt{3 b /(3 b+a) P_{\mathrm{T}}}
\end{array},\right.
$$

where $p_{1}^{(i)}, i=1,2,3$ are the power assigned to the first TA for the $i$ th intersections. Then, based on the fixed total power constraint $P_{\mathrm{T}}$, the corresponding power assigned to the second TA is given by

$$
\left\{\begin{array}{l}
p_{2}^{(1)}=\sqrt{a /(a+b) P_{\mathrm{T}}} \\
p_{2}^{(2)}=\sqrt{3 a /(3 a+b) P_{\mathrm{T}}} \\
p_{2}^{(3)}=\sqrt{a /(3 b+a) P_{\mathrm{T}}}
\end{array}\right.
$$

Based on Eqs. (17) and (18), we select the one providing the maximum $d_{\min }$ as the final solution. Finally, the optimal TPC matrix $\mathbf{U}_{\mathrm{opt}}$ and the corresponding maximized $d_{\mathrm{min}}$, namely $d_{\min }^{\max }$, are given by

$\left\{\begin{array}{lll}\text { if } a \geq b, & \mathbf{U}_{\mathrm{opt}}=\operatorname{diag}\left\{p_{1}^{(2)}, p_{2}^{(2)} e^{j \theta_{1}^{o p t}}\right\}, & d_{\min }^{\max }=\frac{4 a b}{3 a+b} \\ \text { if } a<b, & \mathbf{U}_{\mathrm{opt}}=\operatorname{diag}\left\{p_{1}^{(3)}, p_{2}^{(3)} e^{j \theta_{1}^{o p t}}\right\}, & d_{\min }^{\max }=\frac{4 a b}{3 b+a}\end{array}\right.$

It is worth noting that since the Euclidean distance $d_{\min }^{\max }$ of Eq. (19) has two independent channel gains, a transmit diversity order of two may be achieved [40].

\section{Optimal TPC Matrix for M-PSK-Modulated $\left(2 \times N_{r}\right)$ - Element SM}

In this subsection, the approach proposed in Section III-B is extended to $M$-PSK modulated SM schemes. Based on the method of Section III-B, the max- $d_{\min }$ based TPC algorithm can be summarized as follows:

In order to better illustrate the general algorithm described above, let us consider the specific example of constant-modulus $M$-PSK modulation, whose symbols belong to the set $s \sim \mathfrak{s}=$ $e^{j \frac{2 l \pi}{M}}(l \in\{1, \ldots, M\})$. The minimum distance between two symbols of the $M$-PSK constellation is $d_{M-\mathrm{PSK}}=2 \sin (\pi / M)$ [38]. Since the $\mathrm{SM}$ symbols $\mathbf{x}_{i}$ and $\mathbf{x}_{j}$ only have a single non-zero element, the error vectors $\mathbf{e}_{i j}=\mathbf{x}_{i}-\mathbf{x}_{j}, i \neq j$ can be classified into two types: the error vectors having only a single non-zero element, and those having two non-zero elements. The first type is generated by the transmit symbols $\mathbf{x}_{i}$ and $\mathbf{x}_{j}$ associated with the same TA activation position, while the second type is generated by the symbols having different active TAs. As a result, the distance $d_{i j}(\mathbf{H})$ of Eq. (7) can be divided into two sets: $\mathbb{D}_{1}$ and $\mathbb{D}_{2}$, which are given by

$$
\left\{\begin{array}{l}
\mathbb{D}_{1}=\left\{p_{i}^{2}\left\|\mathbf{h}_{i}\right\|^{2}\left(s_{l}-s_{\hat{l}}\right), l \neq \hat{l}, i=1,2\right\} \\
\mathbb{D}_{2}=\left\{\left\|p_{1} \mathbf{h}_{1} s_{l}-p_{2} \mathbf{h}_{2} s_{\hat{l}}\right\|^{2}, l, \hat{l}=1, \ldots M\right\}
\end{array},\right.
$$

where $s_{l}=e^{j \frac{2 l \pi}{M}}$ and $s_{\hat{l}}=e^{j \frac{2 \hat{l} \pi}{M}}$ are two $M$-PSK symbols. Since only the minimum distance is investigated in the max$d_{\text {min }}$ optimization problem of Eq. (10), only the minimum value
Algorithm 1. The max $-d_{\text {min }}$ based TPC algorithm

Step 1: Compute all legitimate error vectors $\mathbf{e}_{i j}=\mathbf{x}_{i}-\mathbf{x}_{j}, i \neq$ $j$ by eliminating all collinear elements. Calculate all legitimate received distances $d_{i j}(\mathbf{H})$ with the aid of the channel matrix $\mathbf{H}$ and $\mathbf{e}_{i j}$. Let $\mathbb{D}$ be the set of these distances, whose elements are denoted by $d_{v}(v=$ $1, \ldots, V)$, where $V$ is the cardinality of the set $\mathbb{D}$. The set $\mathbb{D}$ is divided into two sub-sets $\mathbb{D}_{1}$ and $\mathbb{D}_{2}$, where $\mathbb{D}_{1}$ contains the error vectors, which have only a single non-zero element, and $\mathbb{D}_{2}$ contains the error vectors, which have two non-zero elements. ${ }^{2}$

Step 2: Find the optimal phase $\theta_{1}^{o p t}$, which maximizes the minimum received distance of the set $\mathbb{D}_{2}$. Note that there may be multiple optimal phase solutions, which are calculated based on shifted sinusoidal functions $d_{v}$ in $\mathbb{D}_{2}$. Since these solutions provide the same $d_{\text {min }}$, any one of them can be randomly selected.

Step 3: After finding the optimal phase $\theta_{1}^{o p t}$, compute all possible intersections between the received distances $d_{i}$ and $d_{j}\left(d_{i}, d_{j} \in \mathbb{D}\right)$ and compute the corresponding PA matrix P. Select the one having the largest $d_{\text {min }}$ as the final PA result, which can be formulated as $\mathbf{P}=\operatorname{diag}\left\{p_{1}^{o p t}, p_{2}^{o p t}\right\}$. Then, the final TPC solution is given by $\mathbf{U}_{\mathrm{opt}}=\operatorname{diag}\left\{p_{1}^{o p t}, p_{2}^{o p t} e^{j \theta_{1}^{o p t}}\right\}$.

of the set $\mathbb{D}_{1}$ has to be considered. To be specific, only the pair 322 of elements $d_{1}=\min _{l \neq \hat{l}} p_{1}^{2}\left\|\mathbf{h}_{1}\right\|^{2}\left(s_{l}-s_{\hat{l}}\right)=d_{M-\operatorname{PSK}} p_{1}^{2}\left\|\mathbf{h}_{1}\right\|^{2} 323$ and $d_{2}=\min _{l \neq \hat{l}} p_{2}^{2}\left\|\mathbf{h}_{2}\right\|^{2}\left(s_{l}-s_{\hat{l}}\right)=d_{M-\mathrm{PSK}} p_{2}^{2}\left\|\mathbf{h}_{2}\right\|^{2}$ has to be 324 considered in $\mathbb{D}_{1}$. Hence, the set $\mathbb{D}_{1}$ is simplified to

$$
\mathbb{D}_{1}=\left\{d_{M-\mathrm{PSK}}^{2} p_{1}^{2}\left\|\mathbf{h}_{1}\right\|^{2}, d_{M-\mathrm{PSK}}^{2} p_{2}^{2}\left\|\mathbf{h}_{2}\right\|^{2}\right\} .
$$

Let us reduce the set $\mathbb{D}_{2}$. When only the phase difference of 326 the PSK symbols $s_{l}=e^{j \frac{2 l \pi}{M}}$ and $s_{\hat{l}}=e^{j \frac{2 \hat{l} \pi}{M}}$ is considered, the 327 set $\mathbb{D}_{2}$ can be modified to

$$
\begin{aligned}
\mathbb{D}_{2} & =\left\{\left\|\mathbf{h}_{1} p_{1} s_{l}-\mathbf{h}_{2} p_{2} e^{j \theta_{1}} s_{\hat{l}}\right\|^{2}, s_{l}, s_{\hat{l}} \in \mathfrak{s}\right\} \\
& =\left\{\left\|p_{1} \mathbf{h}_{1} e^{j \frac{2(l-\hat{l}) \pi}{M}}-p_{2} e^{j \theta_{1}} \mathbf{h}_{2}\right\|^{2}, l, \hat{l}=1, \ldots M\right\} \\
& =\left\{\left\|p_{1} \mathbf{h}_{1} e^{j \frac{2 k \pi}{M}}-p_{2} e^{j \theta_{1}} \mathbf{h}_{2}\right\|^{2}, k=0, \ldots M-1\right\},
\end{aligned}
$$

where the phase difference factor is $k=l-\hat{l}$. The reduction 329 principle behind Eq. (22) is that if the error vectors in the set $\mathbb{D}_{2} \quad 330$ having only a phase difference, they provide the same distance 331 at the receiver. Based on this principle, the number of elements 332 in $\mathbb{D}_{2}$ is reduced to $M$ compared to $M(M-1)$. 333

Let $\lambda_{k}=\frac{2 k \pi}{M}$ be the phase difference of the symbol $s_{l}$ and 334 $s_{\hat{l}}$. Since the distances in the set $\mathbb{D}_{1}$ are independent of the 335 phase $\theta_{1}$, similar to the BPSK case portrayed in Section III-B, 336

\footnotetext{
${ }^{2}$ Note that the transmit vector of SM has only a single non-zero element, hence the number of non-zero elements of the error vectors of SM is up to 2.
} 
it is possible to first find the optimal phase $\theta_{1}^{o p t}$, which maximizes the minimum received distance of the set $\mathbb{D}_{2}$. To achieve this goal, the intersections between arbitrary received distances $\left\|\mathbf{h}_{1} p_{1} e^{j \lambda_{a}}-\mathbf{h}_{2} p_{2} e^{j \theta_{1}}\right\|^{2}$ and $\left\|\mathbf{h}_{1} p_{1} e^{j \lambda_{b}}-\mathbf{h}_{2} p_{2} e^{j \theta_{1}}\right\|^{2}$ in Eq. (22) are firstly calculated as

$$
\begin{gathered}
\left\|\mathbf{h}_{1} p_{1} e^{j \lambda_{a}}-\mathbf{h}_{2} p_{2} e^{j \theta_{1}}\right\|^{2}=\left\|\mathbf{h}_{1} p_{1} e^{j \lambda_{b}}-\mathbf{h}_{2} p_{2} e^{j \theta_{1}}\right\|^{2} \\
\Leftrightarrow\left(c_{1} \cos \lambda_{a}-c_{2} \sin \lambda_{a}-c_{1} \cos \lambda_{b}+c_{2} \sin \lambda_{b}\right) \cos \theta_{1} \\
=-\left(c_{1} \sin \lambda_{a}+c_{2} \cos \lambda_{a}-c_{1} \sin \lambda_{b}-c_{2} \cos \lambda_{b}\right) \sin \theta_{1} \\
\Leftrightarrow \tan \theta_{1}=-\frac{c_{1} \cos \lambda_{a}-c_{2} \sin \lambda_{a}-c_{1} \cos \lambda_{b}+c_{2} \sin \lambda_{b}}{c_{1} \sin \lambda_{a}+c_{2} \cos \lambda_{a}-c_{1} \sin \lambda_{b}-c_{2} \cos \lambda_{b}} .
\end{gathered}
$$

After that, all possible optimal phase $\theta_{1}^{o p t}$ can be obtained from Eq. (23) as

$$
\left\{\begin{array}{c}
\theta_{1}^{o p t}=k \pi+\tan ^{-1}\left(\frac{-c_{1} \cos \lambda_{a}+c_{2} \sin \lambda_{a}+c_{1} \cos \lambda_{b}-c_{2} \sin \lambda_{b}}{c_{1} \sin \lambda_{a}+c_{2} \cos \lambda_{a}-c_{1} \sin \lambda_{b}-c_{2} \cos \lambda_{b}}\right), \\
k \in \mathbb{Z}, 0 \leq \theta_{1}^{o p t} \leq 2 \pi .
\end{array} .\right.
$$

Finally, the optimal phase is the candidate providing the maximum distance $d_{\min }$ in the set of Eq. (24). After computing the optimal phase, the the PA matrix can be optimized based on all possible intersections of $d_{v}(v=1, \ldots, V)$, similar to processes of Eqs. (16)-(18). Following these calculation steps, the optimal TPC matrix, which combines the optimal phase and PA parameters, is obtained in closed-form.

\section{Example for QPSK Modulation}

Based on the algorithm in Section III-C, we calculate the optimal TPC solution for QPSK-modulated $\left(2 \times N_{r}\right)$-element SM, which will be used in our simulations. The symbols of QPSK modulation belong to the set $\mathfrak{s}=\{1,-1, j,-j\}$ and the value of $d_{4-\mathrm{PSK}}$ is equal to $\sqrt{2}$. Based on Eqs. (21) and (22), the corresponding sets $\mathbb{D}_{1}$ and $\mathbb{D}_{2}$ for QPSK modulation are

$$
\left\{\begin{array}{l}
\mathbb{D}_{1}=\left\{2 p_{1}^{2}\left\|\mathbf{h}_{1}\right\|^{2}, 2 p_{2}^{2}\left\|\mathbf{h}_{2}\right\|^{2}\right\} . \\
\mathbb{D}_{2}=\left\{\left\|p_{1} \mathbf{h}_{1} e^{j \frac{2 k \pi}{M}}-p_{2} e^{j \theta_{1}} \mathbf{h}_{2}\right\|^{2}, k=0, \ldots, 3\right\},
\end{array}\right.
$$

According to Eq. (24), there are two optimal phases $\theta_{1}^{o p t}$ that maximizes the distance $d_{\min }$ for Eq. (25), namely $\theta_{1}^{\text {opt }, 1}$ and $\theta_{1}^{o p t, 2}$, which are given by

$$
\left\{\begin{array}{c}
\theta_{1}^{o p t, 1}=k \pi+\tan ^{-1}\left(\frac{-c_{1}-c_{2}}{c_{1}-c_{2}}\right) \\
k \in \mathbb{Z}, 0 \leq \theta_{1}^{o p t, 1} \leq 2 \pi
\end{array}\right.
$$

and

$$
\left\{\begin{array}{c}
\theta_{1}^{o p t, 2}=k \pi+\tan ^{-1}\left(\frac{c_{1}-c_{2}}{c_{1}+c_{2}}\right), \\
k \in \mathbb{Z}, 0 \leq \theta_{1}^{o p t, 2} \leq 2 \pi .
\end{array}\right.
$$

Since both solutions $\theta_{1}^{o p t, 1}$ and $\theta_{1}^{o p t, 2}$ have the same $d_{\min }$, we consider only the first case $\theta_{1}=\theta_{1}^{o p t, 1}$. After finding the optimal phase $\theta_{1}^{o p t, 1}$, the received distance set $\mathbb{D}_{2}$ is further reduced to $\mathbb{D}_{2}=\left\{\left\|p_{1} \mathbf{h}_{1} e^{j \frac{2 k \pi}{M}}-p_{2} e^{j \theta_{1}} \mathbf{h}_{2}\right\|^{2}, k=0,1\right\}$. This reduction 365 is due to the fact that $\theta_{1}^{\text {opt }, 1}$ corresponds to the intersection of 366 two elements of $\mathbb{D}_{2}$ and the elements having the same value are 367 eliminated. After this reduction, the final received distance set 368 has only 4 elements (both $\mathbb{D}_{1}$ and $\mathbb{D}_{2}$ have 2 elements), denoted 369 by $\bar{d}_{i}(i=1,2,3,4)$.

Given the optimal phase as $\theta_{1}^{\text {opt }}=\theta_{1}^{\text {opt }, 1}$, we have to fur- 371 ther identify the optimal PA parameters $p_{1}^{\text {opt }}$ and $p_{2}^{o p t}\left(p_{2}^{\text {opt }}=372\right.$ $\sqrt{\left.P_{\mathrm{T}}-\left(p_{1}^{o p t}\right)^{2}\right)}$. According to the step 3 of Algorithm 1, the 373 max $-d_{\min }$ solution of $p_{1}$ is one of the intersections between 374 these received distance $\bar{d}_{i}(i=1,2,3,4)$, which are given by 375

$$
\left\{\begin{array}{l}
p_{1}^{(1)}=\sqrt{b /(a+b) P_{\mathrm{T}}} \\
p_{1}^{(2)}=p_{1}^{(5)}=\sqrt{\frac{2 c^{2}+4 a b+2 \tilde{C} \sqrt{\tilde{C}^{2}+4 a b}}{4 a^{2}+2 c^{2}+4 a b+2 \tilde{C} \sqrt{\tilde{C}^{2}+4 a b}} P_{\mathrm{T}}} \\
p_{1}^{(3)}=p_{1}^{(4)}=\sqrt{\frac{2 c^{2}+4 a b-2 \tilde{C} \sqrt{\tilde{C}^{2}+4 a b}}{4 a^{2}+2 c^{2}+4 a b-2 \tilde{C} \sqrt{\tilde{C}^{2}+4 a b}} P_{\mathrm{T}}}
\end{array} .\right.
$$

Based on the power constraint, the power allocated on 376 the second $\mathrm{TA}$ is obtained by $p_{2}^{(i)}=\sqrt{P_{\mathrm{T}}-\left(p_{1}^{(i)}\right)^{2}}, i=377$ $1, \ldots, 5$. Finally, the distances $d_{\min }$ of these TPC solutions 378 $\mathbf{U}=\operatorname{diag}\left\{p_{1}^{(i)}, p_{2}^{(i)} e^{j \theta_{1}^{o p t, 1}}\right\}(i=1,2,3,4,5)$ are generated and 379 that having the largest $d_{\min }$ is chosen as our final result $\mathbf{U}_{\text {opt }}$. 380

E. A Low-Complexity Iterative Max- $d_{\min }$ for $N_{t}>2$

It is worth mentioning that the restriction of considering 382 $\left(2 \times N_{r}\right)$-element $\mathrm{SM}$ is imposed by the difficulty of the $d_{\min } 383$ optimization. The solution of the general problem remains an 384 open challenge for two reasons. Firstly, the solution depends on 385 both the channel matrix and on the symbol alphabet, and sec- 386 ondly, the solution space is large. Similar to the general method 387 proposed in the PA aided SM of [33], some sub-optimal meth- 388 ods can be adopted for the case of $N_{t}>2$ based on an iterative 389 process relying on the above-mentioned optimal max- $d_{\min }$ solu- 390 tion provided for $N_{t}=2$, where the TPC algorithm will only 391 be used for the specific TA pair associated with $d_{\min }$, while the 392 parameters of other TAs remain unchanged in each iteration.

\section{Minimum BER BASED TPC}

Although the max- $d_{\min }$ based TPC algorithm is simple, it 395 may not achieve a significant BER improvement for some SM 396 systems, because only one of the distances in the PEP expres- 397 sion of Eq. (6), namely $d_{\text {min }}$, is optimized at the receiver. 398 Moreover, we can only obtain closed-form solutions for this 399 TPC algorithm for the case of $N_{t}=2$. To deal with these prob- 400 lems, we propose a new min-BER based TPC algorithm, which 401 is capable of jointly optimizing all the received distances for 402 directly improving the BER for arbitrary value of $N_{t}$. By con- 403 sidering the bit-to-symbol mapping rule of our SM scheme, a 404 more accurate conditional BER bound based on Eq. (6) can be 405 obtained as [41]

$$
\begin{aligned}
P_{e}(\mathbf{H}) \leq P_{e}^{\mathrm{up}}(\mathbf{H})= & \frac{1}{L} \sum_{\substack{\mathbf{x}_{i} \in \mathbb{X} \\
\mathbf{x}_{\mathbf{x}_{j} \in \mathbb{X}}}} D_{H}\left(\mathbf{x}_{i} \rightarrow \mathbf{x}_{j}\right) \\
& \cdot Q\left(\sqrt{\frac{1}{2 N_{0}}\left\|\mathbf{H U}\left(\mathbf{x}_{i}-\mathbf{x}_{j}\right)\right\|^{2}}\right),
\end{aligned}
$$


where $D_{H}\left(\mathbf{x}_{i} \rightarrow \mathbf{x}_{j}\right)$ is the Hamming distance between the SM signals $\mathbf{x}_{i}$ and $\mathbf{x}_{j}$. From Eq. (29), the min-BER-based TPC matrix is proposed by solving the optimization problem as follows

$$
\begin{aligned}
& \mathbf{U}_{\mathrm{opt}}=\underset{\mathbf{U}}{\arg \min } P_{e}^{\mathrm{up}}(\mathbf{H}) \\
& \text { s.t. } \operatorname{tr}\left(\mathbf{U}^{T}\right)=P_{\mathrm{T}}
\end{aligned}
$$

Remark: Compared to Eq. (30), the $\max -d_{\min }$ based TPC algorithm of Eq. (10) considers only a reduced summation over a subset of $\mathbb{X}$, which has the smallest Euclidean distance. Therefore, it can only minimize a much looser bound of BER than the bound of Eq. (29).

\section{A. Precoder Design Based on Gradient Optimization}

Since the direct solution of Eq. (30) is complex, we drive the theoretical gradient of the cost function with respect to the diagonal TPC matrix $\mathbf{U}$ and invoke the SCG algorithm of [36] for low-complexity TPC matrix optimization. More specifically, the cost function of the SCG algorithm is obtained from Eq. (29) and is defined as

$$
\begin{aligned}
J e(\mathbf{U})= & \sum_{\substack { \mathbf{x}_{i} \in \mathbb{X} \\
\begin{subarray}{c}{\mathbf{x}_{j} \in \mathbb{X} \\
\mathbf{x}_{i} \neq \mathbf{x}_{j}{ \mathbf { x } _ { i } \in \mathbb { X } \\
\begin{subarray} { c } { \mathbf { x } _ { j } \in \mathbb { X } \\
\mathbf { x } _ { i } \neq \mathbf { x } _ { j } } }\end{subarray}} D_{H}\left(\mathbf{x}_{i} \rightarrow \mathbf{x}_{j}\right) \\
& \cdot Q\left(\sqrt{\frac{1}{2 N_{0}}\left\|\mathbf{H U}\left(\mathbf{x}_{i}-\mathbf{x}_{j}\right)\right\|^{2}}\right) .
\end{aligned}
$$

The conjugate gradient of Eq. (31) with respect to $\mathbf{U}$ is given by

$$
\begin{aligned}
\nabla J e(\mathbf{U})= & \frac{-\mathbf{H}^{H} \mathbf{H U}}{4 \sqrt{\pi} N_{0}} \times \sum_{\substack{\mathbf{x}_{i} \in \mathbb{X} \\
\mathbf{x}_{\mathbf{x}_{j} \in \mathbb{X}} \\
\mathbf{x}_{i} \neq \mathbf{x}_{j}}}\left\{D_{H}\left(\mathbf{x}_{i} \rightarrow \mathbf{x}_{j}\right)\right. \\
& \left.\cdot \varphi\left(\mathbf{x}_{i} \rightarrow \mathbf{x}_{j}\right) \cdot \exp \left(-\frac{\varepsilon}{4 N_{0}}\right)\left(\frac{\varepsilon}{4 N_{0}}\right)^{-\frac{1}{2}}\right\},
\end{aligned}
$$

where we have

$$
\begin{aligned}
& \varphi\left(\mathbf{x}_{i} \rightarrow \mathbf{x}_{j}\right)=\left(\mathbf{x}_{i}-\mathbf{x}_{j}\right)\left(\mathbf{x}_{i}-\mathbf{x}_{j}\right)^{H}=\mathbf{e}_{i j} \mathbf{e}_{i j}^{H}, \\
& \varepsilon=\left\|\mathbf{H U}\left(\mathbf{x}_{i}-\mathbf{x}_{j}\right)\right\|^{2} .
\end{aligned}
$$

It is worth noting that the TPC matrix $\mathbf{U}$ is a diagonal matrix, hence the final diagonal conjugate gradient matrix is constituted by the diagonal elements of $\nabla J e(\mathbf{U})$. The derivation of Eq. (32) is given in Appendix A. Given the conjugate gradient of Eq. (32), the problem of Eq. (30) can be solved iteratively by commencing the iterations from an appropriate initial point using the SCG algorithm of [36]. In order to have an initial diagonal TPC matrix $\mathbf{U}^{(1)} \in \mathbb{C}^{N_{t} \times N_{t}}$, we use the max- $d_{\text {min }}$ based TPC matrix solution for $\left(2 \times N_{r}\right)$-element $\mathrm{SM}$ systems and adopt the near-optimal max- $d_{\min }$ solution in Section IV for the other scenarios. ${ }^{3}$ Then, we optimize the TPC matrix with the aid of the SCG algorithm as follows:

\footnotetext{
${ }^{3}$ Moreover, we can also use the equally weighted diagonal matrix or other optimized TPC matrix of [33]-[35] as initial TPC matrix.
}

\section{Algorithm 2. The min-BER based TPC algorithm}

1) Initialization: Set a step size of $\mu>0$, a termination scalar of $\beta>0$ and a maximum number of iterations $N_{\text {all }}$; given the conjugate gradient of the initial diagonal TPC matrix $\mathbf{U}^{(1)}$ as $\tau(1)=\nabla J e\left(\mathbf{U}^{(1)}\right) \in \mathbb{C}^{N_{t} \times N_{t}}$, set $n=1$.

2) Loop: if $\left\|\nabla J e\left(\mathbf{U}^{(1)}\right)\right\|<\beta$ or $n>N_{\text {all }}$, goto Stop.

$$
\begin{aligned}
& \mathbf{U}^{(n+1)}=\mathbf{U}^{(n)}-\mu \tau(n) /\|\tau(n)\|, \\
& \alpha=P_{\mathrm{T}} / \operatorname{tr}\left(\mathbf{U}^{(n+1)}\left(\mathbf{U}^{(n+1)}\right)^{H}\right), \\
& \mathbf{U}^{(n+1)}=\sqrt{\alpha} \mathbf{U}^{(n+1)}, \\
& \varphi_{l}=\left\|\nabla J e\left(\mathbf{U}^{(n+1)}\right)\right\|^{2} /\left\|\nabla J e\left(\mathbf{U}^{(n)}\right)\right\|^{2}, \\
& \tau(n+1)=\varphi_{l} \tau(n)-\nabla J e\left(\mathbf{U}^{(n+1)}\right) .
\end{aligned}
$$

$n=n+1$, goto Loop.

3) Stop: $\mathbf{U}^{(n+1)}$ is the solution.

As shown in [36] and [37], the convergence of the SCG 436 algorithm is more rapid than that of the classic steepest gradient 437 algorithm. For the sake of avoiding convergence to a local opti- 438 mum, the values of $\varphi_{l}$ in SCG can be periodically reset either 439 to zero or to their negative counterparts [36]. ${ }^{4}$

\section{B. SCG Algorithm Based on the Simple Q-function Estimations}

In the SCG algorithm, the computational complexity is 442 dominated by the calculation of the conjugate gradient of 443 Eq. (32). To reduce this complexity, two simple upper 444 bounds of the Gaussian Q-function can be adopted. The 445 first well-known estimate is given by the Chernoff bound as 446 follows [39]

$$
Q(x) \leq \frac{1}{2} \exp \left(-\frac{x^{2}}{2}\right)
$$

Hence, the conjugate gradient of Eq. (32) with respect to $\mathbf{U}$ is 448 simplified to

$$
\begin{gathered}
\nabla J e_{C h e r}(\mathbf{U})=\frac{-\mathbf{H}^{H} \mathbf{H U}}{4 N_{0}} \times \sum_{\substack { \mathbf{x}_{i} \in \mathbb{X} \\
\begin{subarray}{c}{\mathbf{x}_{j} \in \mathbb{X} \\
\mathbf{x}_{i} \neq \mathbf{x}_{j}{ \mathbf { x } _ { i } \in \mathbb { X } \\
\begin{subarray} { c } { \mathbf { x } _ { j } \in \mathbb { X } \\
\mathbf { x } _ { i } \neq \mathbf { x } _ { j } } }\end{subarray}}\left\{D_{H}\left(\mathbf{x}_{i} \rightarrow \mathbf{x}_{j}\right)\right. \\
\left.\cdot \varphi\left(\mathbf{x}_{i} \rightarrow \mathbf{x}_{j}\right) \cdot \exp \left(-\frac{\varepsilon}{4 N_{0}}\right)\right\} .
\end{gathered}
$$

A more accurate approximation of the Q-function than the 450 Chernoff bound is formulated as a sum of weighted expo- 451 nentials. By considering only two components, the following 452 Chiani-bound has been proposed in [39]

$$
Q(x) \leq \frac{1}{12} \exp \left(-\frac{x^{2}}{2}\right)+\frac{1}{4} \exp \left(-\frac{2 x^{2}}{3}\right)
$$

\footnotetext{
${ }^{4}$ Further information about the SCG algorithm is available in [37].
} 


$$
\begin{aligned}
& \text { and the corresponding conjugate gradient } \nabla J e_{C h a i}(\mathbf{U}) \text { is } \\
& \nabla J e_{C h a i}(\mathbf{U})=\frac{-\mathbf{H}^{H} \mathbf{H U}}{4 N_{0}} \cdot \sum_{\substack { \mathbf{x}_{i} \in \mathbb{X} \\
\begin{subarray}{c}{\mathbf{x}_{j} \in \mathbb{X} \\
\mathbf{x}_{i} \neq \mathbf{x}_{j}{ \mathbf { x } _ { i } \in \mathbb { X } \\
\begin{subarray} { c } { \mathbf { x } _ { j } \in \mathbb { X } \\
\mathbf { x } _ { i } \neq \mathbf { x } _ { j } } }\end{subarray}}\left\{D_{H}\left(\mathbf{x}_{i} \rightarrow \mathbf{x}_{j}\right)\right. \\
& \left.\varphi\left(\mathbf{x}_{i} \rightarrow \mathbf{x}_{j}\right) \cdot\left[\frac{1}{6} \exp \left(-\frac{\varepsilon}{4 N_{0}}\right)+\frac{2}{3} \exp \left(-\frac{\varepsilon}{3 N_{0}}\right)\right]\right\} .
\end{aligned}
$$

Eqs. (40) and (42) provide two simple approximations of Eq. (32). It is worth noting that the transmit vectors of SM schemes are sparsely populated, since they have mostly zero values, hence the space of non-linear error vectors $\mathbf{e}_{i j}=\mathbf{x}_{i}$ $\mathbf{x}_{j}$ is small, as shown in Section III. For example, the number of non-linear error vectors $\mathbf{e}_{i j}$ for QPSK-modulated SM associated with $N_{t}=2$ is as low as six. In the SCG-based TPC optimization, we may only have to consider these non-linear error vectors and hence the computational complexity of the SCG algorithm can be further reduced.

\section{Min-BER Based TPC Matrix Design With Imperfect CSI}

In practical applications, pilot symbols are commonly used for estimating the MIMO channel, but naturally the estimated MIMO channel matrix is inevitably imperfect. Hence, the TPC design algorithm should give cognizance to the estimated MIMO channel matrix $\hat{\mathbf{H}}$, which is given by [42], [43]

$$
\hat{\mathbf{H}}=\mathbf{H}+\Delta \mathbf{H},
$$

where $\Delta \mathbf{H}$ is the channel estimation error matrix. Let us assume that $\Delta \mathbf{H}$ is uncorrelated with $\mathbf{H}$ and satisfies $\Delta \mathbf{H}^{H} \Delta \mathbf{H}=$ $\sigma_{e r r}^{2} \mathbf{I}_{N_{t}}$. Then, the corresponding gradient for SCG algorithm is computed as

$$
\begin{aligned}
& \nabla J e(\mathbf{U})=\frac{-\hat{\mathbf{H}}^{H} \hat{\mathbf{H}} \mathbf{U}}{4 \sqrt{\pi}} \cdot \sum_{\mathbf{x}_{i} \in \mathbb{X}} \frac{1}{\sigma_{e}^{2}} \sum_{\substack{\mathbf{x}_{j} \in \mathbb{X} \\
\mathbf{x}_{i} \neq \mathbf{x}_{j}}}\left\{D_{H}\left(\mathbf{x}_{i} \rightarrow \mathbf{x}_{j}\right)\right. \\
& \left.\varphi\left(\mathbf{x}_{i} \rightarrow \mathbf{x}_{j}\right) \exp \left(-\frac{\varepsilon \hat{\mathbf{H}}}{4 \sigma_{e}^{2}}\right)\left(\frac{\varepsilon_{\hat{\mathbf{H}}}}{4 \sigma_{e}^{2}}\right)^{-\frac{1}{2}}\right\}+\frac{\sigma_{e r r}^{2} \mathbf{U}}{4 \sqrt{\pi}} \sum_{\mathbf{x}_{i} \in \mathbb{X}} \frac{\mathbf{x}_{i} \mathbf{x}_{i}^{H}}{\sigma_{e}^{4}} \\
& \quad \cdot \sum_{\mathbf{x}_{j} \in \mathbb{X}} D_{H}\left(\mathbf{x}_{i} \rightarrow \mathbf{x}_{j}\right) \cdot \exp \left(-\frac{\varepsilon_{\hat{\mathbf{H}}}}{4 \sigma_{e}^{2}}\right)\left(\frac{\varepsilon_{\hat{\mathbf{H}}}}{4 \sigma_{e}^{2}}\right)^{-\frac{1}{2}} \varepsilon,
\end{aligned}
$$

where we have

$$
\sigma_{e}^{2}=N_{0}+\left(\mathbf{U} \mathbf{x}_{i}\right)^{H} \Delta \mathbf{H}^{H} \Delta \mathbf{H} \mathbf{U} \mathbf{x}_{i}
$$

and

$$
\varepsilon_{\hat{\mathbf{H}}}=\left\|\hat{\mathbf{H}} \mathbf{U}\left(\mathbf{x}_{i}-\mathbf{x}_{j}\right)\right\|^{2} .
$$

The derivation details of Eq. (44) are given in Appendix. As shown in Eq. (44), the resultant gradient carefully takes the channel estimation errors into account, when constructing the diagonal TPC matrix. Hence, the BER performance becomes resilient to CSI errors.
TABLE I

COMPUTATIONAL COMPLEXITy IMPOSED By THE GRADIENT $\nabla J e(\mathbf{U})$

\begin{tabular}{|l|l|}
\hline & Complexity imposed \\
\hline $\mathbf{H}^{H} \mathbf{H}$ & $O_{1}=N_{t}^{2}\left(2 N_{r}-1\right)$ \\
\hline$\bullet \times \mathbf{U}$ & $O_{2}=N_{t} N_{r}$ \\
\hline$\varphi\left(\mathbf{x}_{i} \rightarrow \mathbf{x}_{j}\right)$ & $O_{3}=N_{t}\left(\begin{array}{l}2 \\
M\end{array}\right)+4\left(\begin{array}{c}2 \\
N_{t}\end{array}\right) M^{2}$ \\
\hline$\varepsilon=\left\|\mathbf{H U}\left(\mathbf{x}_{i}-\mathbf{x}_{j}\right)\right\|^{2}$ & $O_{4}=N_{t}\left(\begin{array}{l}2 \\
M\end{array}\right)\left(2 N_{r}-1\right)+4\left(\begin{array}{c}2 \\
N_{t}\end{array}\right) M^{2}\left(2 N_{r}-1\right)$ \\
\hline
\end{tabular}

\section{Complexity Analysis of the Proposed TPC Algorithms}

In this section, we provide complexity evaluations of the 484 proposed max- $d_{\min }$ based TPC and the min-BER based TPC 485 algorithms, where only the multiplications of complex numbers 486 are considered.

Based on a similar analysis method to that of [33], for the 488 case of $N_{t}=2$, the closed-form solution of the max- $d_{\min }$ based 489 TPC can be found by using the Algorithm 1, which imposes a 490 complexity of

$$
\begin{aligned}
O_{\max -d_{\min }=} & \underbrace{4\left(2 N_{r}-1\right)}_{\text {calculate } \mathbf{H}^{H} \mathbf{H}}+\underbrace{15 M(M-1) / 2}_{\text {calculate optimal phase }} \\
& +\underbrace{(2 M+1)(M+7)}_{\text {calculate optimal PA }} .
\end{aligned}
$$

Moreover, for the case of $N_{t}>2$, an iterative max- $d_{\min }$ based 492 TPC can be adopted and the associated complexity (similar to 493 Eq. (22) of [33]) is

$$
\begin{aligned}
O_{\max }-d_{\min }= & \underbrace{4\left(2 N_{r}-1\right)}_{\text {calculate } \mathbf{H}^{H} \mathbf{H}}+\underbrace{\left(\begin{array}{c}
2 \\
N_{t}
\end{array}\right)(2 M-1)}_{\text {intial } d_{\min }}+\cdots \\
& n_{\mathrm{TPC}}[\underbrace{15 M(M-1) / 2}_{\text {calculate optimal phase }}+\underbrace{(2 M+1)(M+7)}_{\text {calculate optimal PA }} \\
& +\underbrace{2\left(N_{t}-2\right)(2 M-1)}_{\text {optimized } d_{\min }}],
\end{aligned}
$$

where $n_{\text {TPC }}$ is the number of iterations in the max- $d_{\min }$ based 495 TPC algorithm, which is varied according to the channel matrix. 496 In our simulations, we found that the average value of $n_{\mathrm{TPC}}$ is 497 approximated to 5 .

The complexity of the proposed min-BER based TPC algo- 499 rithm can be estimated by considering: (a) the computational 500 complexity of the SCG solution process in each iteration 501 and (b) the number of iterations $n_{\mathrm{SCG}}$ required for approach- 502 ing convergence. The first term can be estimated based on 503 Eqs. (34)-(38). In Table I, we characterize the computational 504 complexity imposed by the gradient $\nabla J e(\mathbf{U})$, where the sparse 505 structure of the SM symbols $\mathbf{x}_{i}, \mathbf{x}_{j}$ and of the diagonal TPC 506 matrix $\mathbf{U}$ are exploited. To be specific, the error vectors $\mathbf{e}_{i j}=507$ $\mathbf{x}_{i}-\mathbf{x}_{j}, i \neq j$ can be classified into two sets: $N_{t}\left(\begin{array}{c}2 \\ M\end{array}\right)$ vectors hav- 508 ing a single non-zero element and $\left(\begin{array}{c}2 \\ N_{t}\end{array}\right) M^{2}$ vectors having two 509 non-zero elements. They have different complexity for the cal- 510 culation of $\varphi\left(\mathbf{x}_{i} \rightarrow \mathbf{x}_{j}\right)$ and $\varepsilon$, as shown in Table I. Note that 511 
(a) $2 \times 2$ QPSK
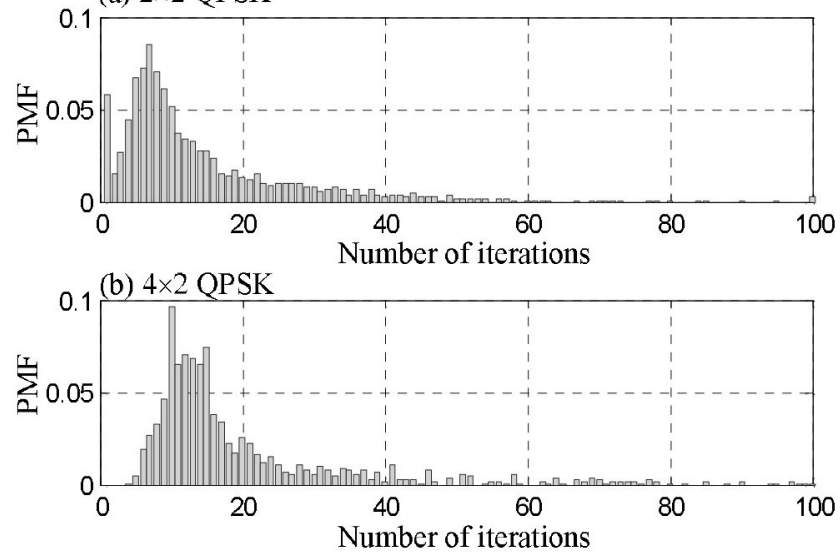

Fig. 3. Convergence behavior of the proposed min-BER based TPC in SM systems with QPSK modulation in i.i.d Rayleigh fading channels.

the bit-to-symbol mapping rule can be designed off-line, hence the complexity of $D_{H}\left(\mathbf{x}_{i} \rightarrow \mathbf{x}_{j}\right)$ is not considered in the calculation of $\nabla J e(\mathbf{U})$. Based on Table I and the SCG algorithm of Eqs. (34)-(38), the associated complexity of the proposed min-BER based TPC algorithm is approximately

$$
\begin{gathered}
O_{\text {min }- \text { BER }}=O_{1}+O_{3}+n_{\mathrm{SCG}}[5\left(O_{2}+O_{4}\right)+\underbrace{N_{t}^{2}+N_{t}}_{\text {Eq. } 34} \\
+\cdots \underbrace{N_{t}^{2}-N_{t}+1}_{\text {Eq } .35}+\underbrace{N_{t}}_{\text {Eq. } 36}+\underbrace{N_{t}^{2}-N_{t}+1}_{\text {Eq. } 37}+\underbrace{2 N_{t}}_{\text {Eq. } 38}]
\end{gathered}
$$

Moreover, similar to [30], in Fig. 3 we have portrayed the probability mass function (PMF) of the numbers of iterations for the min-BER based TPC algorithm in the QPSK-modulated $(2 \times 2)$ and $(4 \times 2)$ SM schemes. In the simulations, the threshold of SCG is given by $\beta=10^{-5}$ and 25000 trails are considered to show the statistics of convergence. In Fig. 3 (a) and (b), more than $90 \%$ and $85 \%$ of the trials converged within 30 iterations. This is due to the rapid convergence of the SCG algorithm, as also verified in [37]. Note that although the approximation method of Section IV-B can reduce the complexity of calculating $\nabla \mathrm{Je}(\mathbf{U})$, i.e. the complexity terms $\mathrm{O}_{3}$ and $\mathrm{O}_{4}$ in Table I, it has the same complexity order as (49). We will provide more detailed comparisons and discussions about the complexity issue in Section VI-C.

\section{Simulation Results}

In this section, we provide simulation results (the distance $d_{\min }$ and the BER performance) for characterizing the max$d_{\min }$ based TPC aided SM and the min-BER based TPC aided SM schemes for transmission over frequency-flat fading channels. For comparison, these performance results are compared to various adaptive SM schemes, such as the ASM arrangements of [21], the maximum minimum distance (MMD) aided SM schemes of [30], the PA-based SM schemes of [33], the
TPC star-QAM SM schemes of [31], and the PRP aided SM 540 schemes of [29] and [34], [35]. $\quad 541$

In the min-BER based TPC scheme, the step size $\mu$ is deter- 542 mined by Monte Carlo simulation methods, as suggested in 543 [36] and we set $\mu=0.01$, so that we achieve a rapid conver- 544 gence, while maintaining excellent BER results. Moreover, for 545 the BPSK case, we do not consider the ASM scheme because 546 'no-transmission' is assigned to one of the TAs and hence this 547 TA is inactive [33].

\section{A. $d_{\min }$ Performance for Different SM Schemes}

In Fig. 4, we compare the complementary cumulative distri- 550 bution functions (CCDF) of the distance $d_{\min }$ recorded for both 551 for conventional SM and for the link adaptive SM schemes in 552 $(2 \times 1)$ MIMO channels under different throughputs. First, we 553 note that these adaptive SM schemes are capable of beneficially 554 increasing the distance $d_{\min }$. As formally shown in Section III, 555 we observe in Fig. 4 that the proposed $\max -d_{\min }$ based TPC 556 aided SM achieves the highest distance $d_{\min }$ compared to other 557 link-adaptive SM schemes. Furthermore, we note that the min- 558 BER based TPC schemes achieve lower $d_{\min }$ than the max- $d_{\min } \quad 559$ based TPC schemes, and yet we will see in Figs. 5-7 that the 560 min-BER based TPC outperforms the max- $d_{\min }$ based TPC in 561 terms of its BER.

\section{B. BER Comparisons of Different SM Schemes}

In Fig. 5, we compare the BER performance of various SM 564 systems for $L=2$ bits/symbol in $(2 \times 1)$ - and $(2 \times 2)$-element 565 MIMO channels. We can see that the proposed min-BER based 566 schemes provides gains of about $6 \mathrm{~dB}$ and $4 \mathrm{~dB}$ at the BER of 567 $10^{-3}$ over the conventional SM schemes. We also confirm that 568 the min-BER based schemes outperform the ASM of [21], the 569 max $-d_{\min }$ based PA aided SM of [33] and the max $-d_{\min }$ based 570 TPC aided SM proposed.

Note that, as shown in Fig. 4, although the optimal max- 572 $d_{\min }$ based TPC aided SM is capable of achieving a higher 573 distance $d_{\min }$ than the other adaptive SM schemes, it does 574 not achieve a BER performance improvement over them. To 575 expound a little further, in Fig. 5, when the proposed max- $d_{\min } 576$ based TPC-aided SM is compared to its special case, namely 577 to the max- $d_{\min }$ based PA aided SM, we find that an increase 578 of the distance $d_{\min }$ by TPC does not achieve any further BER 579 improvement. Observe in Fig. 5 that at high SNRs, the max- 580 $d_{\min }$ based TPC aided SM may even perform worse than the 581 $\max -d_{\min }$ based PA aided SM. This is mainly due to the fact that 582 the maximum of $d_{\min }$ does not necessarily minimize the PEP 583 bound of Eq. (29), which depends on all the received distances. 584

To be specific, the reason for the trends of Fig. 5 is that the 585 max- $d_{\min }$ based TPC may achieve a lower Euclidean distance 586 between the non-adjacent received constellation points than 587 that of the PA schemes. Hence, based on the Q-function aided 588 PEP upper bound of Eq. (29), which depends on all legitimate 589 received distances $d_{i j}(\mathbf{H})=\left\|\mathbf{H U}\left(\mathbf{x}_{i}-\mathbf{x}_{j}\right)\right\|(i \neq j)$, the max- 590 $d_{\min }$ based TPC fails to achieve the best BER performance. For 591 example, let us consider the $(2 \times 1)$ SM scheme using BPSK. 592 As shown in Section III, we only have four different distances 593 

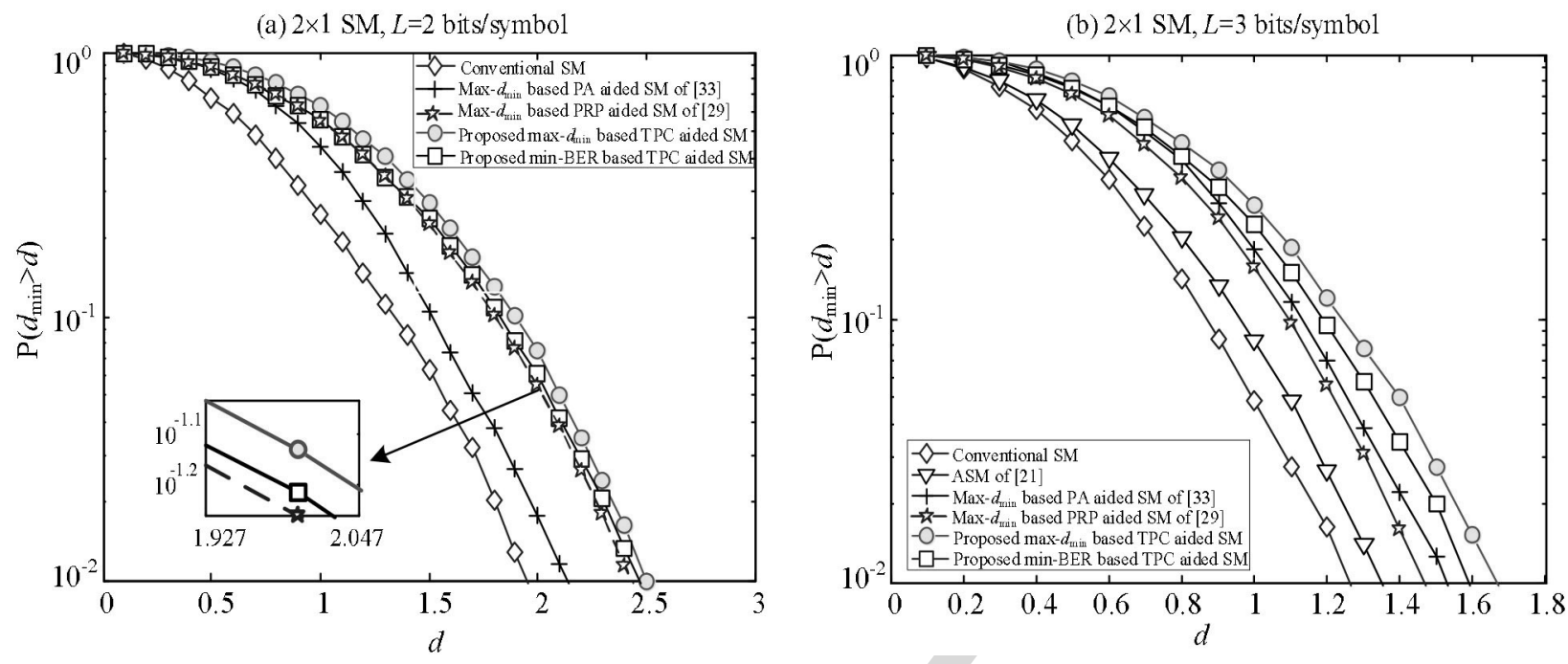

Fig. 4. The CCDF of the minimum distance $d_{\min }$ of conventional SM and of various link-adaptation aided SM schemes in $(2 \times 1)$-element MIMO channels.

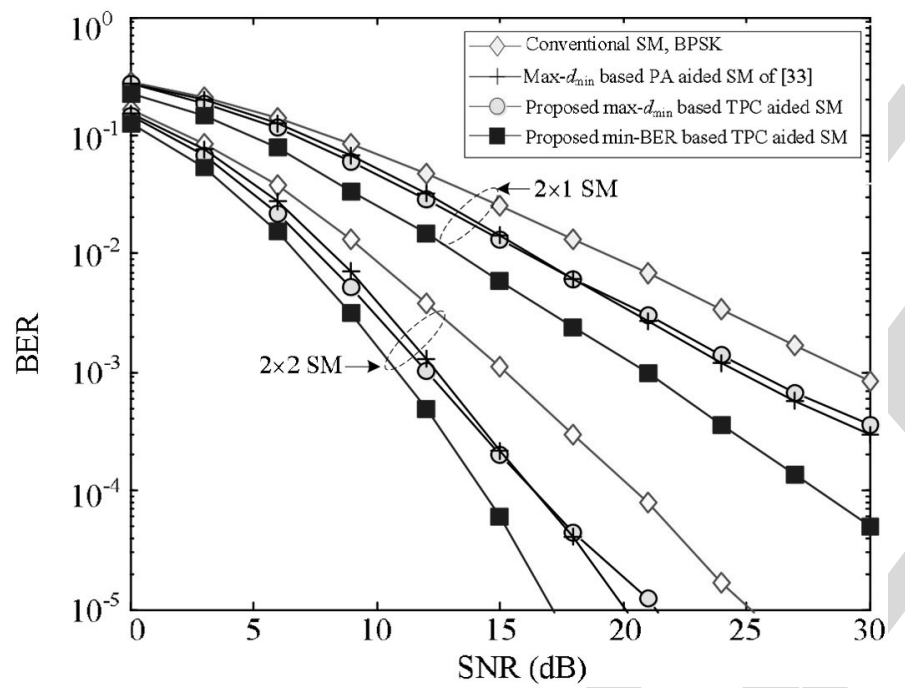

Fig. 5. BER comparison at $L=2$ bits/symbol for the conventional SM, for the max- $d_{\text {min }}$ based TPC aided SM and for the min-BER based TPC aided SM.

at the receiver, namely $d_{1}, d_{2}, d_{3}$ and $d_{4}$. Assuming that the channel matrix is $\mathbf{H}=[0.056-0.069 i, 0.414+1.267 i]$ and the SNR is $25 \mathrm{~dB}$, the resultant distance set for the $\max -d_{\min }$ based TPC-aided SM is

$\mathbb{D}_{T P C}=\left\{d_{1}=0.251, d_{2}=0.434, d_{3}=0.251, d_{4}=0.251\right\}$,

while the corresponding distance set for the $\max -d_{\min }$ based PA aided SM is

$$
\mathbb{D}_{P A}=\left\{d_{1}=0.248, d_{2}=0.718, d_{3}=0.476, d_{4}=0.248\right\} .
$$

Then, the BER results of Eq. (29) calculated for the TPC-aided SM and the PA-aided SM schemes are $P_{e}(\mathbf{H})=0.7 \times 10^{-3}$ and $P_{e}(\mathbf{H})=0.5 \times 10^{-3}$, respectively. This result confirms that although the max $-d_{\min }$ based TPC algorithm achieves the highest distance of $d_{\min }=0.251$, while the $\max -d_{\min }$ based PA algorithm has $d_{\min }=0.248$, the former has a worse PEP performance due to its lower values of $d_{2}$ and $d_{3}$. This result is consistent with the result seen in Fig. 5.

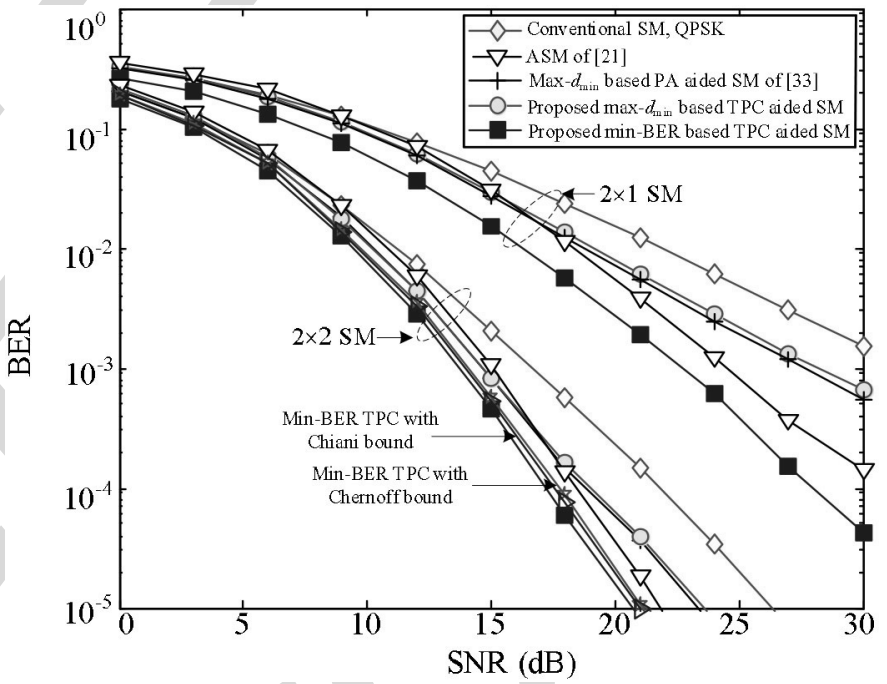

Fig. 6. BER comparison at $L=3$ bits/symbol for various SM schemes. Here, the Q-function estimates of Section IV-B are only considered for the $(2 \times 2)$ element MIMO channels.

The above-mentioned trends of these proposed TPC algo- 608 rithms are also visible in Fig. 6, where the throughput is $L=3609$ bits/symbol. It is shown in Fig. 6 that the proposed min-BER 610 based TPC outperforms both ASM of [21] and the max- $d_{\min } 611$ based PA of [33]. Moreover, in Fig. 6, we demonstrate that the 612 approximate Chernoff-based and Chiani-based optimizations 613 perform almost the same as the exact Q-function based scheme. 614 This is because these approximations do not change the direc- 615 tion of the gradient. We have also simulated the Chernoff-based 616 and Chiani-based optimizations for the other MIMO setups 617 considered, and obtained similar results, as evidenced by Fig. 6. 618 Since the resultant curves approximately overlap with the opti- 619 mal one, for clarity, these results are not included in other 620 figures.

Due to the advantage of the proposed min-BER based TPC, 622 in Fig. 7 we further investigate its performance for a higher 623 number of TAs and modulation order. All the schemes are 624 


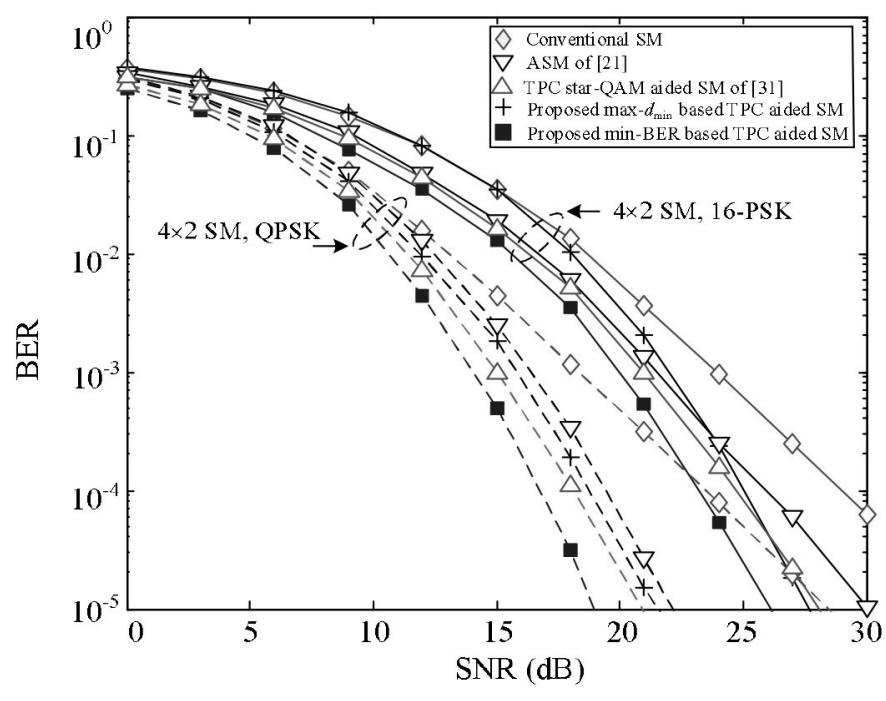

Fig. 7. BER comparison of the proposed min-BER-based TPC-aided SM schemes over the TPC star-QAM aided SM schemes.

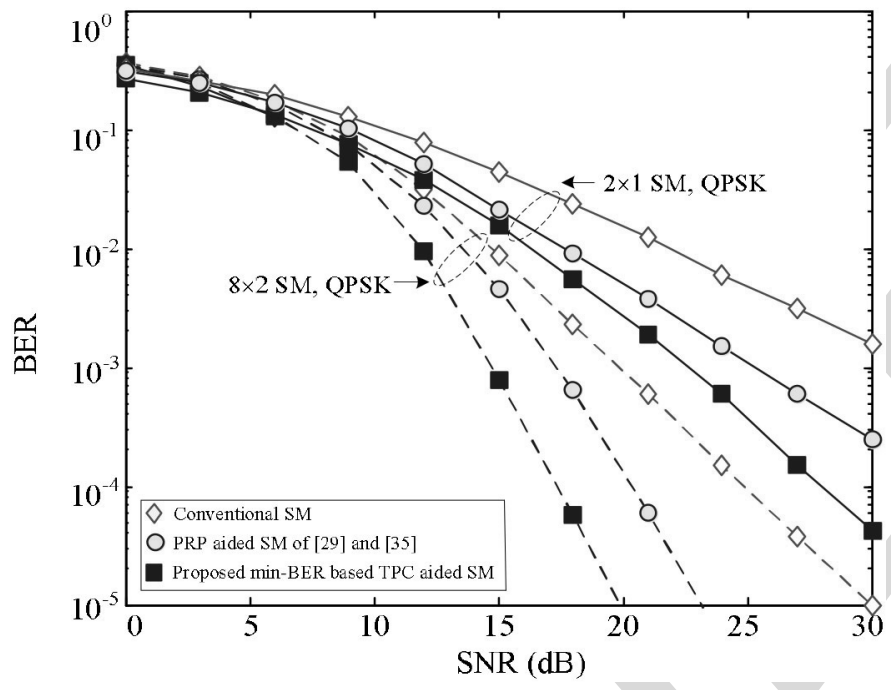

Fig. 8. BER comparison of the proposed min-BER-based TPC-aided SM schemes over the PRP-aided SM schemes. assumed to have $N_{t}=4, N_{r}=2$ and the throughputs are $L=4$ and $L=6$ bits/symbol. In Fig. 7, the proposed schemes are also compared to the TPC star-QAM aided SM schemes of [31], which utilize a quantized search for optimizing the diagonal TPC matrix. In our simulation, the number of quantization levels for both amplitude and phase in TPC of [31] is 6. Observe in Fig. 7 that the proposed min-BER based TPC schemes achieve the best BER performance. The performance gain of the proposed scheme over the TPC star-QAM aided SM scheme is seen to be about $2.6 \mathrm{~dB}$ at $\mathrm{BER}=10^{-5}$ for 4 bits/symbol transmissions in Fig. 7. This is due to the fact the TPC star-QAM based scheme of [31] also only optimizes a single received distance $d_{\text {min }}$, which may limit the attainable BER performance.

In Fig. 8, we compared the proposed min-BER based TPC schemes to the max- $d_{\text {min }}$ based PRP schemes of [29] and [35]. We observe in Fig. 8 that the proposed schemes outperform the PRP-aided schemes. To be specific, as seen in Fig. 8 the

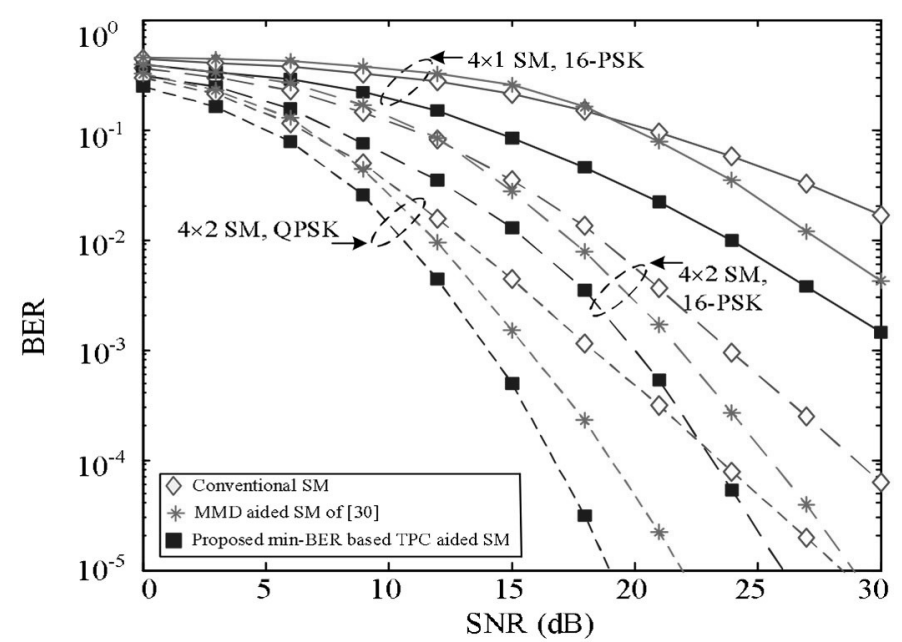

Fig. 9. BER comparison of the proposed min-BER based TPC aided SM schemes over the MMD aided SM schemes.

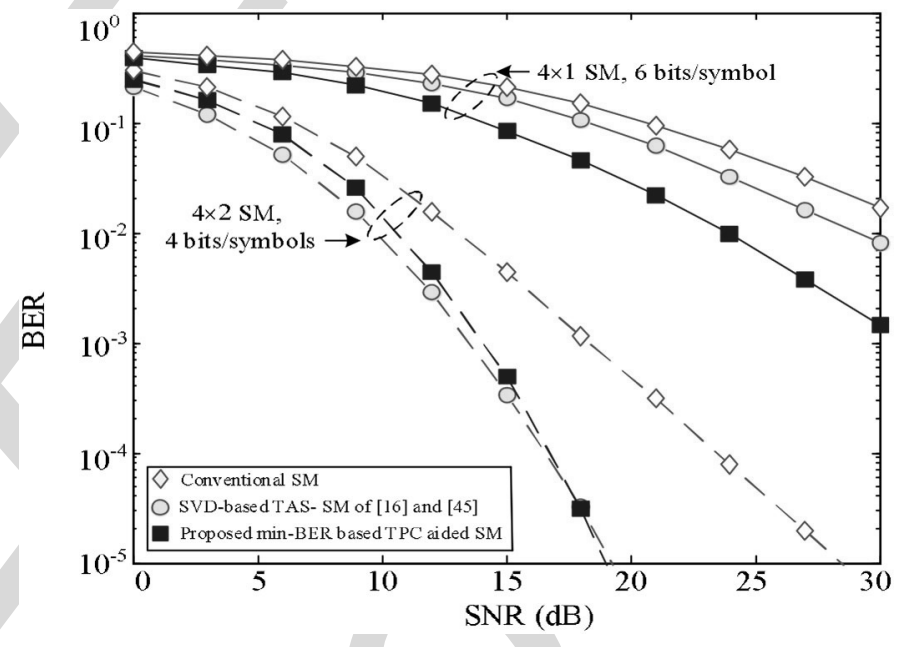

Fig. 10. BER performance of the proposed min-BER-based TPC-aided SM schemes and the SVD-based TAS-SM schemes.

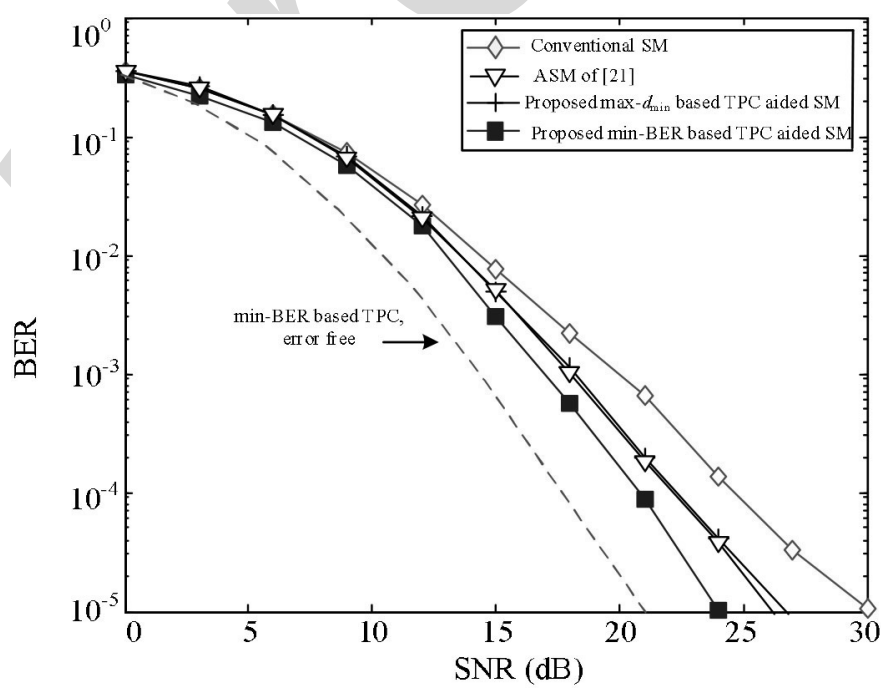

Fig. 11. BER performance of different adaptive SM schemes in the presence of CSI errors. Here, the error variance is $\sigma_{\text {err }}^{2}=1 / r$, where $r$ is the average SNR at each receiver antenna. 
TABLE II

COMPLEXITy ORders For DifFERENT TPC DESigns. THE EXAMPLES ARE With PARAMETERS $n_{\mathrm{SCG}}=30, n_{\mathrm{PA}}=2, n_{\mathrm{PRP}}=3, n_{\mathrm{TPC}}=5, L_{1}=6 \mathrm{AND} L_{2}=6$

\begin{tabular}{|l|l|l|l|}
\hline Designs & Complexity order & $\begin{array}{l}\text { Configuration 1 } \\
(4 \times 2, \mathrm{QPSK})\end{array}$ & $\begin{array}{l}\text { Configuration 2 } \\
(4 \times 2 \text { 16PSK })\end{array}$ \\
\hline ASM of [21] & $\mathcal{O}\left(N_{t}^{2} M^{2}\right)+\mathcal{O}\left(N_{t}^{2} N_{r}\right)$ & 320 & 4160 \\
\hline Max- $d_{\min }$ based PRP of [34] & $\mathcal{O}\left(N_{t}^{2} M\right)+\mathcal{O}\left(n_{\mathrm{PRP}}\left(M^{2}+N_{t} M\right)\right)$ & 160 & 1216 \\
\hline Max- $d_{\min }$ based PA of [33] & $\mathcal{O}\left(N_{t}^{2} M\right)+\mathcal{O}\left(n_{\mathrm{PA}}\left(M^{2}+N_{t} M\right)\right)$ & 128 & 896 \\
\hline Diagonal TPC method of [32] & $\mathcal{O}\left(L_{1} L_{2} N_{t}^{2} M^{2} N_{r}\right)$ & 18432 & 294912 \\
\hline MMD based TPC of [30] & $\mathcal{O}\left(N_{t}^{2} M^{2} N_{r}\right)+\mathcal{O}\left(N_{t}^{4} M^{4}\right)$ & 66048 & 16785408 \\
\hline Proposed max- $d_{\min }$ based TPC & $\mathcal{O}\left(N_{t}^{2} M\right)+\mathcal{O}\left(n_{\mathrm{TPC}}\left(M^{2}+N_{t} M\right)\right)$ & 224 & 1856 \\
\hline Proposed min-BER based TPC & $\mathcal{O}\left(n_{\mathrm{SCG}} N_{t}^{2} M^{2} N_{r}\right)$ & 15360 & 245760 \\
\hline
\end{tabular}

proposed TPC scheme provides about $3.2 \mathrm{~dB}$ gain over the PRP scheme at BER $=10^{-5}$ for $(8 \times 2)$-element MIMO channels at a throughput of 5 bits/symbol. This benefit is due to the following two reasons: (1) the PRP schemes only adapt the phases of the SM symbols and hence the degrees of freedom utilized for TPC design are limited [35]; (2) similar to the methods of [31], [33], they are designed based on the max- $d_{\min }$ principle and hence may provide suboptimal BER.

In Fig. 9, the proposed min-BER based TPC schemes are compared to the MMD-aided SM schemes of [30]. Observe in Fig. 9 that the proposed TPC scheme provides an SNR gain of about $3 \mathrm{~dB}$ over the MMD-aided scheme at the BER of $10^{-5}$ for the $(4 \times 2)$ MIMO channels considered. Similar to the results in [30], the MMD-based TPC schemes provide minor performance improvements or even degrade the performance in low-SNR regimes. This is because the MMD criterion based TPC design may be ineffective in low SNR regimes, as discussed in [30].

Moreover, in Fig. 10, the proposed min-BER based TPC schemes are compared to the TAS-based SM schemes [16], [17], [44], [45] under different throughputs. In Fig. 10, the singular value decomposition (SVD)-based TAS algorithm of [16], [44], [45] is utilized due to its low-complexity and attractive performance. The number of TAs is $N_{t}=4$ and 2 out of $N_{t}=4$ TAs are selected by the TAS algorithm. Without loss of generality, we consider a PSK signal constellation diagram. As shown in Fig. 10, the TAS and the TPC schemes exhibit different BER advantages for different system setups. Specifically, the proposed TPC scheme outperforms the TAS scheme for $(4 \times 1)$ MIMO channels having a throughput of 6 bits/symbol, while they achieve a similar BER performance for $(4 \times 2)$ MIMO associated with 4 bits/symbol. This is not surprising, since the TAS and the TPC algorithms rely on different transmit parameters for the sake of achieving BER improvements. Note that TPC can be added on top of TAS to further improve performance. Hence they are complementary rather than competitive. It has been shown in [24], [30] that the joint design of TPC and TAS can further improve the system performance.

Fig. 11 shows the BER performance of various SM schemes in the presence of Gaussian-distributed CSI errors obeying eN $\left(0, \sigma_{e r r}^{2}\right)$ [42], [43] for $(2 \times 2)$ MIMO channels and $L=3$ bits/symbol. For the sake of simplification and clarity, we only consider the ASM and PA-aided SM schemes as benchmarks. In this paper, the variable $\sigma_{e r r}^{2}$, i.e. the value of the estimation error is adjusted according to the SNR. To be specific, $\sigma_{e r r}^{2}=1 / r$ is 687 adopted, where $r$ is the average SNR at each receiver antenna. 688 As expected, the BER performance of all SM schemes degrades 689 upon imposing CSI estimation errors. However, Fig. 11 shows 690 that the performance degradation of the proposed min-BER 691 based TPC-aided SM is lower than that of the other schemes 692 due to the fact that its BER upper bound was optimized bearing 693 in mind the CSI error by using the SCG algorithm.

\section{Complexity Comparison for Different TPC Designs}

In Table II, the complexity orders of different TPC designs 696 are compared. Specifically, in the randomly-selected diagonal 697 TPC method of [31], [32], the quantization levels of ampli- 698 tude and phase are $L_{1}$ and $L_{2}$, respectively. Its complexity 699 order is provided in [30]. Moreover, the complexity orders of 700 the max $-d_{\min }$ based PRP and of the $\max -d_{\min }$ based PA algo- 701 rithms can be found in [34] and [33], where their iteration 702 numbers are $n_{\mathrm{PA}}$ and $n_{\mathrm{PRP}}$, respectively. In Table II, we also 703 provide the approximate quantified complexity for some spe- 704 cific configurations, where the QPSK- and 16PSK-modulated 705 $4 \times 2$ SM schemes are considered. The number of iterations for 706 the proposed SCG method is set to be $n_{\mathrm{SCG}}=30$ due to it fast 707 convergence.

As shown in Table II, the proposed max $-d_{\min }$ based TPC 709 has a similar complexity order to that of the max- $d_{\min }$ based 710 PRP of [34] and to that of the PA of [33], while exhibiting 711 a lower complexity than the proposed min-BER design, since 712 these $\max -d_{\min }$ based designs only have to optimize a single 713 distance $d_{\min }$. However, as shown in our simulation results of 714 Figs 5-7 these max $-d_{\min }$ based designs suffer from a BER per- 715 formance loss. The MMD-based TPC of [30] is a generalized 716 max $-d_{\text {min }}$ based TPC, which has to optimize $N_{t} M$ TPC weights 717 for all legitimate SM symbols rather than relying on a diago- 718 nal TPC matrix having only $N_{t}$ non-zero elements. Hence, the 719 MMD-based TPC imposes a higher complexity than the pro- 720 posed max- $d_{\min }$ and min-BER based TPC algorithms, as shown 721 in Table II. For example, in configuration 2, the complexity of 722 the proposed min-BER based design approximately achieves 68723 times smaller than that of the MMD-based design.

Moreover, the diagonal TPC method of [32] requires an 725 exhaustive search over a set of $L_{1} L_{2}$ candidates, hence a higher 726 complexity is imposed compared to the proposed min-BER 727 based TPC for a high number of quantization levels. By tak- 728 ing into account both the BER versus complexity trends, we 729 


$$
\varepsilon_{\hat{\mathbf{H}}}=\left\|\hat{\mathbf{H}} \mathbf{U}\left(\mathbf{x}_{i}-\mathbf{x}_{j}\right)\right\|^{2} .
$$

761 Note that $\sigma_{e}^{2}$ and $\varepsilon_{\hat{\mathbf{H}}}$ are functions of the TPC matrix $\mathbf{U}$. Then 762

conclude that the proposed min-BER based TPC provides an improved BER performance at a modest complexity cost. It should be noted that the extra complexity is imposed by the calculation of the gradient $\nabla \mathrm{Je}(\mathbf{U})$ and by the convex problem solution algorithm, which may be further reduced by exploiting the spatial-domain sparsity of SM symbols and with the aid of reduced-complexity solution techniques. This issue will be investigated in our future studies.

\section{CONCLUSIONS}

We have investigated two types of diagonal TPC design algorithms. For the max- $d_{\text {min }}$ based TPC algorithm, closed-form solutions were derived for the case of two TAs and suboptimal solutions were achieved by using iterative method. For the min-BER based TPC algorithm, an iterative SCG algorithm was proposed for finding the specific TPC matrix solution. Finally, the proposed min-BER based TPC algorithm was further enhanced by taking into account the effects of imperfect CSI. It is shown from simulation results that the proposed max$d_{\text {min }}$ based TPC algorithm is optimal in terms of the minimum received distance, while the proposed min-BER based TPC algorithm is optimal in terms of the BER. Our further work will be focused on the integration of space-time coding, channel coding and TAS techniques into the proposed schemes.

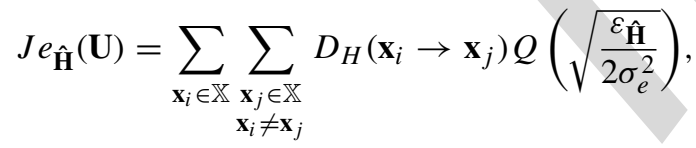

$$
\sigma_{e}^{2}=N_{0}+\sigma_{e r r}^{2}\left(\mathbf{U} \mathbf{x}_{i}\right)^{H} \mathbf{U} \mathbf{x}_{i}
$$

the gradient of $J e_{\hat{\mathbf{H}}}(\mathbf{U})$ can be expressed as:

$$
\begin{aligned}
\nabla J e_{\hat{\mathbf{H}}}(\mathbf{U}) & =\frac{\partial J e_{\hat{\mathbf{H}}}(\mathbf{U})}{\partial \mathbf{U}^{H}} \\
& =\sum_{\mathbf{x}_{i} \in \mathbb{X}} \sum_{\substack{\mathbf{x}_{j} \in \mathbb{X} \\
\mathbf{x}_{i} \neq \mathbf{x}_{j}}} D_{H}\left(\mathbf{x}_{i} \rightarrow \mathbf{x}_{j}\right) \frac{\partial Q\left(\sqrt{\frac{\varepsilon \hat{\mathbf{H}}}{2 \sigma_{e}^{2}}}\right)}{\partial \mathbf{U}^{H}} .
\end{aligned}
$$

Here, $\partial Q\left(\sqrt{\frac{\varepsilon_{\hat{\mathbf{H}}}}{2 \sigma_{e}^{2}}}\right) / \partial \mathbf{U}^{H}$ of Eq. (53) can be expressed as

$$
\begin{aligned}
& \frac{\partial Q\left(\sqrt{\frac{\varepsilon_{\mathbf{H}}}{2 \sigma_{e}^{2}}}\right)}{\partial \mathbf{U}^{H}}=-\frac{1}{\sqrt{\pi}} e^{-\frac{\varepsilon_{\mathbf{H}}}{4 \sigma_{e}^{2}}}\left(\frac{\varepsilon_{\hat{\mathbf{H}}}}{4 \sigma_{e}^{2}}\right)^{-\frac{1}{2}} \frac{\partial\left(\sqrt{\frac{\varepsilon_{\hat{\mathbf{H}}}}{4 \sigma_{e}^{2}}}\right)}{\partial \mathbf{U}^{H}} \\
& =-\frac{1}{\sqrt{\pi}} e^{-\frac{\varepsilon_{\hat{\mathbf{H}}}}{4 \sigma_{e}^{2}}}\left(\frac{\varepsilon_{\hat{\mathbf{H}}}}{4 \sigma_{e}^{2}}\right)^{-\frac{1}{2}}\left\{\left(\frac{\partial\left(\varepsilon_{\hat{\mathbf{H}}}\right)}{\partial \mathbf{U}^{H}} \frac{1}{4 \sigma_{e}^{2}}\right)+\left(\frac{\partial\left(\frac{1}{4 \sigma_{e}^{2}}\right)}{\partial \mathbf{U}^{H}} \varepsilon_{\hat{\mathbf{H}}}\right)\right\},
\end{aligned}
$$

where we have the relationship of

$$
\begin{aligned}
\frac{\partial\left(\varepsilon_{\hat{\mathbf{H}}}\right)}{\partial \mathbf{U}^{H}} & =\hat{\mathbf{H}}^{H} \hat{\mathbf{H}} \mathbf{U} \varphi\left(\mathbf{x}_{i} \rightarrow \mathbf{x}_{j}\right), \\
\frac{\partial\left(\frac{1}{4 \sigma_{e}^{2}}\right)}{\partial \mathbf{U}^{H}} & =-\frac{\sigma_{e r r}^{2} \mathbf{U} \mathbf{x}_{i} \mathbf{x}_{i}^{H}}{4 \sigma_{e}^{4}} .
\end{aligned}
$$

Based on Eq. (56), we can arrive at the gradient matrix of 765 Eq. (44). Moreover, assuming that the CSI is perfectly known, 766 we have $\Delta \mathbf{H}=0, \hat{\mathbf{H}}=\mathbf{H}$ and $\varepsilon_{\hat{\mathbf{H}}}=\varepsilon=\left\|\mathbf{H U}\left(\mathbf{x}_{i}-\mathbf{x}_{j}\right)\right\|^{2} .767$ Then, the gradient matrix of Eq. (32) is readily obtained.

\section{REFERENCES}

[1] R. Y. Mesleh, H. Haas, S. Sinanovic, C. W. Ahn, and S. Yun, "Spatial 770 modulation," IEEE Trans. Veh. Technol., vol. 57, no. 4, pp. 2228-2241, 771 Jul. 2008.

[2] M. Di Renzo, H. Haas, A. Ghrayeb, S. Sugiura, and L. Hanzo, "Spatial 773 modulation for generalized MIMO: Challenges, opportunities and imple- 774 mentation," Proc. IEEE, vol. 102, no. 1, pp. 56-103, Jan. 2014.

[3] S. Sugiura, S. Chen, and L. Hanzo, "A universal space-time architecture 776 for multiple-antenna aided systems," IEEE Commun. Surveys \& Tuts., 777 vol. 14, no. 2, pp. 401-420, May 2012.

[4] E. Başar, Ü. Aygölü, E. Panayırc1, and H. V. Poor, "Space-time block 779 coded spatial modulation," IEEE Trans. Commun., vol. 59, no. 3, pp. 823- 780 832, Mar. 2011.

[5] M. Di Renzo, H. Haas, and P. M. Grant, "Spatial modulation for multiple- 782 antenna wireless systems: A survey," IEEE Commun. Mag., vol. 49, 783 no. 12, pp. 182-191, Dec. 2011.

[6] M. Di Renzo and H. Haas, "Bit error probability of SM-MIMO over 785 generalized fading channels," IEEE Trans. Veh. Technol., vol. 61, no. 3, 786 pp. 1124-1144, Mar. 2012.

[7] A. Stavridis, S. Sinanovic, M. Di Renzo, and H. Haas, "Energy evaluation 788 of spatial modulation at a multi-antenna base station," in Proc. IEEE Veh. 789 Technol. Conf., Barcelona, Spain, Sep. 2013, pp. 1-5.

[8] N. Serafimovski et al. "Practical implementation of spatial modulation" 791 IEEE Trans. Veh. Technol., vol. 62, no. 9, pp. 511-523, Nov. 2013.

[9] J. Jeganathan, A. Ghrayeb, and L. Szczecinski, "Spatial modulation: 793 Optimal detection and performance analysis," IEEE Commun. Lett., 794 vol. 12 , no. 8, pp. 545-547, Aug. 2008.

[10] S. Sugiura, C. Xu, S. X. Ng, and L. Hanzo, "Reduced complexity coherent 796 versus non-coherent QAM-aided space time shift keying," IEEE Trans. 797 Commun., vol. 59, no. 11, pp. 3090-3101, Nov. 2011.

[11] N. R. Naidoo, H. Xu, and T. Quazi, "Spatial modulation: Optimal detec- 799 tor asymptotic performance and multiple-stage detection," IET Commun., 800 vol. 5, no. 10, pp. 1368-1376, Jul. 2011.

[12] A. Younis, S. Sinanovic, M. Di Renzo, R. Y. Mesleh, and H. Haas, 802 "Generalised sphere decoding for spatial modulation," IEEE Trans. 803 Commun, vol. 61, no. 7, pp. 2805-2815, Jul. 2013.

[13] R. Y. Chang, S.-J. Lin, and W.-H. Chung, "Energy efficient transmis- 805 sion over space shift keying modulated MIMO channels," IEEE Trans. 806 Commun., vol. 60, no. 10, pp. 2950-2959, Oct. 2012.

[14] S. Sanayei and A. Nosratinia, "Antenna selection in MIMO systems," 808 IEEE Commun. Mag., vol. 42, no. 10, pp. 68-73, Oct. 2004.

[15] D. J. Love, R. W. Heath Jr., U. K. N. Lau, D. Gesbert, B. D. Rao, and 810 M. Andrews, "An overview of limited feedback in wireless communica- 811 tion systems," IEEE J. Sel. Areas Commun., vol. 26, no. 8, pp. 1341-1365, 812 Oct. 2008

[16] K. Ntontin, M. Di Renzo, A. Perez-Neira, and C. Verikoukis, "A low- 814 complexity method for antenna selection in spatial modulation systems," 815 IEEE Commun. Lett., vol. 17, no. 12, pp. 2312-2315, Aug. 2013. 
[17] R. Rajashekar, K. V. S. Hari, and L. Hanzo, "Antenna selection in spatia modulation systems," IEEE Commun. Lett., vol. 17, no. 3, pp. 521-524, Mar. 2013.

[18] N. Pillay and H. Xu, "Comments on "Antenna selection in spatial modulation systems'," IEEE Commun. Lett., vol. 17, no. 9, pp. 1681-1683, Sep. 2013.

[19] P. Yang, Y. Xiao, Y. Yi, and S. Li, "Adaptive spatial modulation for wireless MIMO transmission systems," IEEE Commun. Lett., vol. 15, no. 6, pp. 602-604, Jun. 2011.

[20] P. Yang, Y. Xiao, L. Li, Q. Tang, Y. Yu, and S. Li, "Link adaptation for spatial modulation with limited feedback," IEEE Trans. Veh. Technol., vol. 61, no. 8, pp. 3808-3813, Oct. 2012.

[21] P. Yang, Y. Xiao, Y. Yi, L. Li, Q. Tang, and S. Q. Li, "Simplified adaptive spatial modulation for limited-feedback MIMO," IEEE Trans. Veh. Technol., vol. 62, no. 2, pp. 2656-2666, Jul. 2013.

[22] R. Rajashekar, K. V. S. Hari, and L. Hanzo, "Reduced-complexity ML detection and capacity-optimized training for spatial modulation," IEEE Trans. Commun., vol. 62, no. 1, pp. 112-125, Jan. 2014.

[23] K. Ntontin, M. Di Renzo, A. I. Perez-Neira, and C. Verikoukis, "Adaptive generalized space shift keying," EURASIP J. Wireless Commun. Netw. vol. 43, Feb. 2013.

[24] M. Maleki, H. R. Bahrami, S. Beygi, M. Kafashan, and N. H. Tran, "Space modulation with CSI: Constellation design and performance evaluation," IEEE Trans. Veh. Technol., vol. 64, no. 4, pp. 1623-1634, May 2013.

[25] M. Maleki, H. R. Bahrami, M. Kafashan, and N. H. Tran, "On the performance of spatial modulation: Optimal constellation breakdown," IEEE Trans. Commun., vol. 62, no. 1, pp. 144-157, Jan. 2014.

[26] C. B. Chae, A. Forenza, R. W. Heath Jr., M. R. Mckay, and I. B. Collings, "Adaptive MIMO transmission techniques for broadband wireless communication systems," IEEE Commun. Mag., vol. 48, no. 5, pp. 112-118, May 2010.

[27] L. Collin, O. Berder, P. Rostaing, and G. Burel, "Optimal minimum distance-based precoder for MIMO spatial multiplexing systems," IEEE Trans. Signal Process., vol. 52, no. 5, pp. 617-627, Mar. 2004.

[28] M. Di Renzo and H. Haas, "Improving the performance of space shift keying (SSK) modulation via opportunistic power allocation," IEEE Commun. Lett., vol. 14, no. 6, pp. 500-502, Jun. 2010.

[29] C. Masouros, "Improving the diversity of spatial modulation in MISO channels by phase alignment," IEEE Commun. Lett., vol. 18, no. 5, pp. 729-732, May 2014.

[30] M. Lee, W. Chung, and T. Lee, "Generalized precoder design formulation and iterative algorithm for spatial modulation in MIMO systems with CSIT," IEEE Trans. Wireless Commun., vol. 63, no. 4, pp. 1230-1244, Apr. 2015.

[31] P. Yang, Y. Xiao, B. Zhang, S. Li, M. El-Hajjar, and L. Hanzo, "StarQAM signaling constellations for spatial modulation," IEEE Trans. Veh. Technol., vol. 63, no. 8, pp. 3741-3749, Oct. 2014.

[32] P. Yang, M. Di Renzo, Y. Xiao, S. Li, and L. Hanzo, "Design guidelines for spatial modulation," IEEE Commun. Surveys Tuts., vol. 17, no. 1, pp. 6-26, Mar. 2015

[33] P. Yang, Y. Xiao, S. Li, and L. Hanzo, "A low-complexity power allocation algorithm for multiple-input multiple-output spatial modulation systems," IEEE Trans. Veh. Technol., 2015, to be published, doi: 10.1109/TVT.2015.2410252.

[34] C. Masouros and L. Hanzo, "Constellation-randomization achieves transmit diversity for single-RF spatial modulation," IEEE Trans. Veh. Technol., 2015, to be published.

[35] P. Yang, Y. Xiao, S. Li, and L. Hanzo, "Phase rotation based transmit precoding improves the minimum Euclidean distance of single-RF-chain aided spatial modulation," IET Commun., 2015, to be published.

[36] M. Bazaraa, H. Sherali, and C. Shetty, Nonlinear Programming: Theory and Algorithms. Hoboken, NJ, USA: Wiley, 1993.

[37] S. Chen, N. Ahmad, and L. Hanzo, "Adaptive minimum bit-error rate beamforming," IEEE Trans. Wireless Commun., vol. 4, no. 2, pp. 341348, Mar. 2005.

[38] J. Proakis, Digital Communications. New York, NY, USA: McGraw-Hill, 2001

[39] A. Goldsmith, Wireless Communications. Cambridge, U.K.: Cambridge Univ. Press, 2005.

[40] D. Yang, C. Xu, L.-L. Yang, and L. Hanzo, "Transmit diversity-assisted space-shift keying for collocated and distributed/cooperative MIMO elements," IEEE Trans. Veh. Technol., vol. 60, no. 6, pp. 2864-2869, Jul. 2011.

[41] T. Handte, A. Muller, and J. Speidel, "BER analysis and optimization of generalized spatial modulation in correlated fading channels," in Proc. IEEE Veh. Technol. Conf., Anchorage, AK, USA, Sep. 2009, pp. 1-5.
[42] E. Basar, Ü. Aygölü, E. Panayırcı, and H. V. Poor, "Performance of spatial 894 modulation in the presence of channel estimation errors," IEEE Commun. 895 Lett., vol. 16, no. 2, pp. 176-179, Feb. 2012.

[43] S. S. Ikki and R. Mesleh, "A general framework for performance analy- 897 sis of space shift keying (SSK) modulation in the presence of Gaussian 898 imperfect estimations," IEEE Commun. Lett., vol. 16, no. 2, pp. 228-230, 899 Feb. 2012

[44] N. Wang, W. Liu, H. Men, M. Jin, and H. Xu. "Further complexity reduc- 901 tion using rotational symmetry for EDAS in spatial modulation," IEEE 902 Commun. Lett., vol. 18, no. 10, pp. 1835-1838, Oct. 2014.

[45] J. Zheng and J. Chen, "Further complexity reduction for antenna selec- 904 tion in spatial modulation systems," IEEE Commun. Lett., vol. 19, no. 6, 905 pp. 937-940, Jun. 2015, doi: 10.1109/LCOMM.2015.2417884.

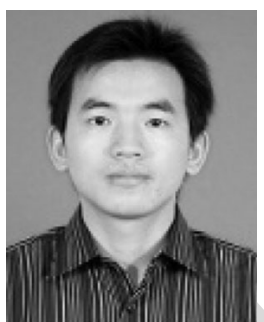

Ping Yang received the B.E. and M.E. degrees 907 from the University of Electronic Science and 908 Technology of China (UESTC), Chengdu, China, in 909 2006 and 2009, respectively. From September 2012910 to September 2013, he was a Visiting Student at 911 the School of Electronics and Computer Science, 912 University of Southampton, Southampton, U.K. 913 From May 2014, he is a Research Fellow with 914 EEE, NTU, Singapore. Also, he is an Assistant 915 Professor with UESTC. His research interests include 916 MIMO/OFDM, machine learning, life science, and 917 communication signal processing.

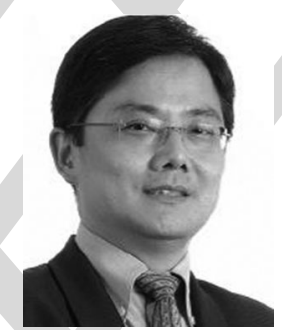

storage systems.

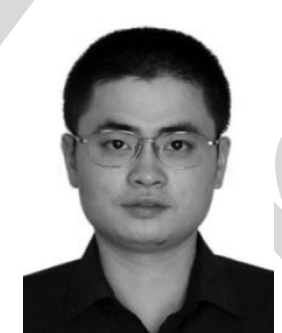

Yong Liang Guan (S'94-AM'97-M'99) received 919 the Ph.D. degree from the Imperial College of 920 Science, Technology and Medicine, University of 921 London, London, U.K., and the B.Eng. degree (with 922 first class Hons.) from the National University of 923 Singapore, Singapore, in 1997 and 1991, respectively. 924 $\mathrm{He}$ is now an Associate Professor with the School 925 of Electrical and Electronic Engineering, Nanyang 926 Technological University, Singapore. His research 927 interests include modulation, coding and signal pro- 928 cessing for communication, information security, and 929

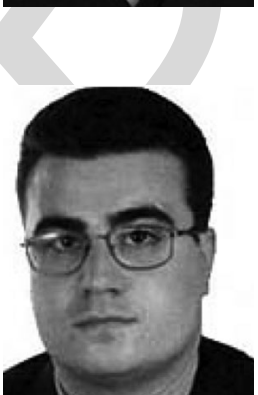

Yue Xiao received the Ph.D. degree in communi- 931 cation and information systems from the University 932 of Electronic Science and Technology of China, 933 Chengdu, China, in 2007. He is now a Full Professor 934 with the University of Electronic Science and 935 Technology of China. He has authored more than 30936 international journals and been involved in several 937 projects in the Chinese Beyond 3G Communication 938 R\&D Program. His research interests include wire- 939 less and mobile communications.

Marco Di Renzo (S'05-AM'07-M'09-SM'14) was 941 born in L'Aquila, Italy, in 1978. He received the 942 Laurea (cum laude) and Ph.D. degrees in electri- 943 cal and information engineering from the University 944 of L'Aquila, L'Aquila, Italy, in April 2003 and 945 January 2007, respectively. Since January 2010, he 946 has been a Tenured Academic Researcher ("Chargé 947 de Recherche Titulaire") with the French National 948 Center for Scientific Research (CNRS), as well as 949 a Faculty Member of the Laboratory of Signals and 950 Systems (L2S), a joint Research Laboratory of the 951 CNRS, the École Supérieure d'Électricité (SUPÉLEC) and the University of 952 Paris-Sud XI, Paris, France. His research interests include wireless communi- 953 cations theory. Currently, he serves an Editor of the IEEE COMMUNICATIONS 954 LETTERS and the IEEE TRANSACTIONS ON COMMUNICATIONS. He is the 955 recipient of a special mention for the 2012 IEEE CAMAD Best Paper Award; 956 IEEE-COMSOC Best Young Researcher Award for Europe, Middle East and 958 Africa (EMEA Region); and the 2014 IEEE ICNC Single Best Paper Award 959 Nomination (Wireless Communications Symposium). the 2013 Network of Excellence NEWCOM\# Best Paper Award; the 2013957

\section{(1) ,

.

.


961

962

963

964

965

966

967

968

969

970

971

972

973

974

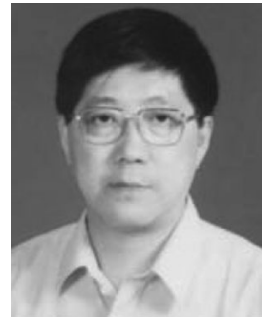

Shaoqian Li (AM'04-SM'12) received the B.S.E. degree in communication technology from Northwest Institute of Telecommunication, Xidian University, Xidian, China, and the M.S.E. degree in communication system from the University of Electronic Science and Technology of China (UESTC), Chengdu, China, in 1982 and 1984, respectively. He is a Professor, Ph.D Supervisor, Director of the National Key Laboratory of Communication, UESTC, and Member of the National High Technology R\&D Program (863 Program) Communications Group. His research interests include wireless communication theory, antiinterference technology for wireless communications, spread-spectrum and frequency-hopping technology, mobile, and personal communications.

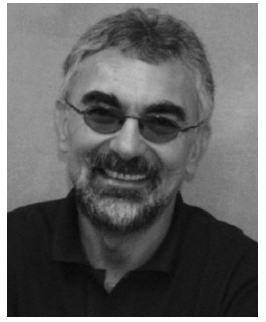

Lajos Hanzo (M'91-SM'92-F'04) received the 975 D.Sc. degree in electronics and the doctorate degree, 976 in 1976 and 1983, respectively. During his 37-year 977 career in telecommunications, he has held various 978 research and academic posts in Hungary, Germany, 979 and the U.K. Since 1986, he has been with the School 980 of Electronics and Computer Science, University of 981 Southampton, Southampton, U.K, where he holds 982 the Chair in Telecommunications. He has success- 983 fully supervised 80+ Ph.D. students, coauthored 20984 Wiley/IEEE Press books on mobile radio communi- 985 cations totaling in excess of 10000 pages, authored 1400+ research entries at 986 IEEE Xplore, acted both as TPC and General Chair of the IEEE conferences, 987 presented keynote lectures and has been awarded a number of distinctions. 988 Currently, he is directing a 100-strong academic research team, working on 989 a range of research projects in the field of wireless multimedia communica- 990 tions sponsored by industry, the Engineering and Physical Sciences Research 991 Council (EPSRC) U.K., the European Research Council's Advanced Fellow 992 Grant and the Royal Society's Wolfson Research Merit Award. He is also a 993 Governor of the IEEE VTS. From 2008 to 2012, he was the Editor-in-Chief of 994 the IEEE Press and a Chaired Professor also at Tsinghua University, Beijing. 995 His research is funded by the European Research Council's Senior Research 996 Fellow Grant. He served as FREng, FIEEE, FIET, a Fellow of EURASIP. In 997 2009, he was the recipient of the honorary doctorate "Doctor Honoris Causa" 998 by the Technical University of Budapest, Budapest, Hungary. 


\section{QUERIES}

Q1: Please be advised that per instructions from the Communications Society this proof was formatted in Times Roman font and therefore some of the fonts will appear different from the fonts in your originally submitted manuscript. For instance, the math calligraphy font may appear different due to usage of the usepackage[mathcal]euscript. We are no longer permitted to use Computer Modern fonts.

Q2: Please provide page range for Ref. [23].

Q3: Please provide volume number, issue number, and page range for Refs. [33], [34], and [35]. 